\title{
The Complexity of Embedded Axiomatization for a Class of Closed Database Views ${ }^{\dagger}$
}

\author{
Stephen J. Hegner \\ Umeå University \\ Department of Computing Science \\ SE-901 87 Umeå, Sweden \\ hegnerdcs. umu.se \\ http://www.cs.umu.se/ hegner
}

Revised: 3 September 2005

\begin{abstract}
It is well known that the complexity of testing the correctness of an arbitrary update to a database view can be far greater than the complexity of testing a corresponding update to the main schema. However, views are generally managed according to some protocol which limits the admissible updates to a subset of all possible changes. The question thus arises as to whether there is a more tractable relationship between these two complexities in the presence of such a protocol. In this paper, this question is addressed for closed update strategies, which are based upon the constant-complement approach of Bancilhon and Spyratos. The approach is to address a more general question - that of characterizing the complexity of axiomatization of views, relative to the complexity of axiomatization of the main schema. For schemata constrained by denial or consistency constraints, that is, statements which rule out certain situations, such as the equalitygenerating dependencies (EGDs) or, more specifically, the functional dependencies (FDs) of the relational model, a broad and comprehensive result is obtained in a very general framework which is not tied to the relational model in any way. It states that every such schema is governed by an equivalent set of constraints which embed into the component views, and which are no more complex than the original set. For schemata constrained by generating dependencies, of which tuple-generating dependencies (TGDs) in general and, more specifically, both join dependencies (JDs) and inclusion dependencies (INDs) are examples within the relational model, a similar result is obtained, but only within a context known as meet-uniform decompositions, which fails to recapture some important situations.

To address the all-important case of relational schemata constrained by both FDs and INDs, a hybrid approach is also developed, in which the general theory regarding denial constraints is blended with a focused analysis of a special but very practical subset of the INDs known as fanout-free unary inclusion dependencies (fanout-free UINDs), to obtain results parallel to the above-mentioned cases: every such schema is governed by an equivalent set of constraints which embed into the component views, and which are no more complex than the original set. In all cases, the question of view update complexity is then answered via a corollary to this main result.
\end{abstract}

\footnotetext{
${ }^{\dagger}$ Parts of this paper are based upon work reported in [Heg04b]
} 


\section{Introduction}

In a seminal work [BS81], Bancilhon and Spyratos showed how well-behaved update strategies for database views can be modelled in a very general framework using the so-called constant complement strategy. Despite the classical nature of this work, it has seen signifi cant recent application; for example, in [FGM*04], these ideas are applied to problems in the synchronization of tree-structured data.

The theory of constant-complement update strategies has also been advanced in recent years. In [Heg02] and [Heg04a], it is shown that by augmenting this basic framework with natural order structure, true uniqueness for so-called order-based updates may be obtained, in the sense that there is but one way to represent an update to the view in terms of an update to the main schema, regardless of the choice of complement.

In addition to the questions of which updates to a view to allow, and how to reflect those updates back to the main schema, the question of tractability is also of fundamental importance. Specifi cally, it is important to know how diffi cult it is to determine whether a proposed view update is admissible under the constraints inherited from the main schema. Issues surrounding this complexity question are the focus of study in this paper.

1.1 Example - A simple view with complex constraints The complexity of the constraints which govern a view can be much greater than those of the schema over which the view is taken, as the following simple example illustrates. Let $\mathbf{E}_{1}$ be the single-relation schema with relation name $R[A B C D]$ on four attributes, constrained by the set $\mathcal{F}_{1}=\{A \rightarrow D, B \rightarrow D, C D \rightarrow A\}$ of functional dependencies (FDs). For a relation $M$ not to satisfy the constraints of $\mathcal{F}_{1}$, it must fail to satisfy at least one of the FDs, and this may be determined by identifying a specific pair of tuples in $M$ for which that FD fails. In other words, to check whether $M$ satisfi es the constraints of $\mathcal{F}_{1}$, it suffi ces to test each pair of tuples in $M$. Thus, such testing has complexity bound $O\left(n^{2}\right)$ for (worst-case) time, with $n$ the size of the relation. ${ }^{1}$

Now consider the simple projection view $\Pi_{A B C}$ on $\mathbf{E}_{1}$, which maps any relation $M$ on $R[A B C D]$ to its projection $\pi_{A B C}(M)$ on $R[A B C]$. As shown in Appendix A, this view is not fi nitely axiomatizable. More precisely, for any odd positive integer $k$, there is a ternary relation $M_{k}$, containing exactly $k$ tuples, with the property that there is no relation $N$ on $R[A B C D]$ which satisfi es the dependencies in $\mathcal{F}_{1}$ and for which $\pi_{A B C}(N)=M_{k}$, yet for every proper subset $M^{\prime} \subsetneq M_{k}$, there is a relation $N^{\prime}$ on $R[A B C D]$ which satisfi es the dependencies in $\mathcal{F}_{1}$ and for which $\pi_{A B C}\left(N^{\prime}\right)=M^{\prime}$. In other words, it is not possible to test, in time $O\left(n^{k}\right)$ for any fi nite $k$, whether a given ternary relation $M$ is of the form $\pi_{A B C}(N)$ for some relation $N$ on $R[A B C D]$ which satisfi es the constraints of $\mathcal{F}_{1}$.

While this example shows that checking the legality of a candidate state of a view is not a universally easy one, it does not establish that it is universally diffi cult either. Testing an arbitrary proposed update to a view for correctness is far more general a task than is testing a proposed update under a disciplined update strategy. A simple example, based upon 1.1, makes this clear.

\footnotetext{
${ }^{1}$ It is of course possible to do better in certain cases via the use of appropriate data structures. Indeed, upon using the number of disk accesses as the measure of complexity, key constraints may be checked in constant time [WG92]. However, the focus of this paper is a general theory based only upon the size of databases; the nuances of such special cases will not be considered.
} 
1.2 Example - The complexity of view update Let $\mathbf{E}_{2}$ be the relational schema with the fi veattribute relation $S[A B C D E]$, constrained by $\mathcal{F}_{2}=\mathcal{F}_{1} \cup\{A \rightarrow E\}$. The pair of projections $\left\{\Pi_{A B C D}, \Pi_{A B C E}\right\}$, with the obvious semantics, forms a lossless decomposition, since the FD $A \rightarrow E$ implies $A B C \rightarrow E$, which implies the join dependency $\bowtie[A B C E, A B C D]$ [PDGV89, Thm. 3.7]. Thus, $\Pi_{A B C D}$ is a complement of $\Pi_{A B C E}$ in the classical sense of [BS81]. The decomposition into these two views is furthermore dependency preserving, since $\mathcal{F}_{1}$ embeds in $\Pi_{A B C D}$, while $\{A \rightarrow E\}$ embeds in $\Pi_{A B C E}$. Now consider updating the view $\Pi_{A B C E}$ while holding $\Pi_{A B C D}$ constant. All constraints in $\mathcal{F}_{1}$ will be satisfi ed after the update, since these constraints embed in $\Pi_{A B C D}$. Thus, to guarantee that a the new relation $M$ on $S[A B C E]$ is legal, it suffi ces to check that it satisfi es the single constraint $A \rightarrow E$. This observation is critical because $\Pi_{A B C E}$ is not fi nitely axiomatizable, for the same reason that $\mathbf{E}_{1}$ of 1.1 is not. The knowledge that the complement is held constant while performing updates is critical in keeping the complexity within bounds.

The above example notwithstanding, it is reasonable to ask why it is desirable to characterize the constraints within the views themselves. It is quite possible simply to take a proposed update to the view, reflect is back into the main schema using the constant-complement strategy, and accept it iff the resulting state of the main schema satisfi es all of the constraints. The complexity of testing an update in this fashion depends only upon properties of the constraints on the main schema, and not upon those of the views. For example, if the main schema is constrained by FDs (as is $\mathbf{E}_{2}$ in 1.2), then the complexity of testing admissibility of a view update is $O\left(n^{2}\right)$, with $n$ the number of tuples in the main schema. (Note that a join must be computed to check update correctness in this way, but the time complexity to construct it is also $O\left(n^{2}\right)$.)

The goal of the work reported here, as well as that of the preceding work [Heg04a], [Heg02], [Heg90a], is to investigate view update strategies which are closed. Roughly speaking, this means that updating a view should be no different than updating a main schema. The constraints on the view, as well as the updates which are allowed, must be understandable within the context of the view alone. In particular, it must not be necessary to consult the complementary view (or, equivalently, the main schema) to determine whether a view update is admissible. Clearly, the strategy of reflecting the view update back to the main schema, and performing the test for correctness there, is not closed.

While not all situations involving view updates demand closed strategies, it is certainly reasonable to expect that many will. The ideas surrounding this topic, with many illustrating examples, are developed in detail in [Heg04a, Sec. 1], There it is argued that closed update strategies have further desirable properties; more precisely, they are precisely those which are free of update anomalies.

A pair of views which supports a closed update strategy is called a meet complementary pair. In this case, there is a view which is common to these two, called the meet, and it is necessary and suffi cient to hold this meet constant to ensure that updates respect the constant-complement strategy. The details are rather technical; they are developed in great detail in [Heg04a, Sec. 2], and summarized in 2.1 of this paper. It is, however, possible to give a rather simple characterization within a common relational context. Specifi cally, if $R[\mathbf{U}]$ is a relational schema constrained by a set of full dependencies, and $\mathbf{W}_{1}, \mathbf{W}_{2} \subseteq \mathbf{U}$, then $\left\{\Pi_{\mathbf{W}_{1}}, \Pi_{\mathbf{W}_{2}}\right\}$, the pair of views defi ned by the projections of $R[\mathbf{U}]$ onto these attributes, forms a meet-complementary pair iff the associated decomposition is both lossless and dependency preserving, in the classical sense [Heg04a, 2.17]. Furthermore, the meet in this case is just $\Pi_{\mathbf{W}_{1} \cap \mathbf{W}_{2}}$, the projection onto the common column of the two views. An example will help illustrate. 
1.3 Example - Closed and non-closed update strategies Continue with the example $\mathbf{E}_{2}$ of 1.2. The set $\left\{\Pi_{A B C D}, \Pi_{A B C E}\right\}$ forms a meet complementary pair, since the decomposition is both lossless and dependency preserving. The meet of these views is $\Pi_{A B C D \cap A B C E}=\Pi_{A B C}$. The theory of closed updates ensures that to check whether an update to $\Pi_{A B C E}$ under constant complement $\Pi_{A B C D}$ satisfi es the constraints, it suffi ces to check whether it holds $\Pi_{A C}$ constant (in addition to verifying that it satisfi es the local view constraints - in this case $\{A \rightarrow E\}$ ). Note that $\Pi_{A B C}$ may be regarded as a view of $S[A B C E]$; thus, whether the update is admissible does depends only upon the state of $S[A B C E]$, and not upon the specifi c state of $S[A B C D]$. In other words, the test for admissibility of an update to $\Pi_{A B C E}$ may be checked entirely within that view, provided that it is known that the current state of $\Pi_{A B C D}$ is legal.

Now, let $\mathbf{E}_{3}$ have the same fi ve-attribute relation $S[A B C D E]$, but with the additional constraint $D \rightarrow E$. That is, the constraint set is $\mathcal{F}_{3}=\mathcal{F}_{2} \cup\{D \rightarrow E\}$. This decomposition is not dependency preserving (although it is lossless), since $D \rightarrow E$ cannot be embedded in either view. Furthermore, the associated update strategy on $\Pi_{A B C E}$ with constant complement $\Pi_{A B C D}$ is not closed. Indeed, let $M_{1}=\left\{\left(a_{1}, b_{1}, c_{1}, d_{1}, e_{1}\right),\left(a_{2}, b_{2}, c_{2}, d_{2}, e_{1}\right)\right\}$, and let $M_{2}=\left\{\left(a_{1}, b_{1}, c_{1}, d_{1}, e_{1}\right),\left(a_{2}, b_{2}, c_{2}, d_{1}, e_{1}\right)\right\}$, Clearly, each is a legal state of $\mathbf{E}_{3}$. In each case, the associated state of $\Pi_{A B C E}$ is $N=\left\{\left(a_{1}, b_{1}, c_{1}, e_{1}\right)\right.$, $\left.\left(a_{2}, b_{2}, c_{2}, e_{1}\right)\right\}$. Consider the update to $N$ which changes $\left(a_{1}, b_{1}, c_{1}, e_{1}\right)$ to $\left(a_{1}, b_{1}, c_{1}, e_{2}\right)$. This update is legal if the state of $\mathbf{E}_{3}$ is $M_{1}$, but not if it is $M_{2}$. Thus, the admissibility of this proposed update depends upon not only the state of $\Pi_{A B C E}$, but also on the state of the complement $\Pi_{A B C D}$. Hence, the associated strategy is not closed.

The theory developed in this paper includes the decomposition of $\mathbf{E}_{2}$ into $\Pi_{A B C D}$ and $\Pi_{A B C E}$, but excludes the same decomposition of $\mathbf{E}_{3}$.

These examples illustrate that the question of the complexity of checking the correctness of candidate view updates with respect to a closed update strategy may be answered by studying a more general problem - that of determining the complexity of the constraints in an embedded cover (into the views of the decomposition) for the constraints of the main schema. It is primarily this latter question which forms the topic of investigation in this paper. From these examples, it might appear that the answer to this question is a rather trivial one, since the constraints which embed into the views are precisely those which are specifi ed on the main schema. However, this presupposes that the constraints on the main schema are already expressed in a fashion which allows them to be embedded into the views. With FDs, this is not a problem, since it is easy to determine when an FD embeds into a projection (although in general it is necessary to determine whether some cover of the FDs embed [PDGV89, Def. 3.7]), but with more general families of constraints, particularly those which generate new elements, the representation of the constraints on the main schema as an equivalent family of constraints which embed in the views is far from trivial. Furthermore, there is no guarantee that this re-representation of full constraints as embedded ones can be achieved with no change of complexity.

The main results of this paper are along two dimensions. For schemata constrained by rules which check consistency (e.g., the equality generating dependencies (EGDs) [PDGV89, Sec. 3.6] of the classical relational theory), a result is established, in a very general set-based context, independently of any particular data model, which states that there is an embedded cover of those constraints into the views which is no more complex than the original constraints. On the other hand, for situations in which rules which generate new elements are allowed, (e.g., the tuple-generating dependencies (TGDs) of the classical relational theory, including the implicational dependencies and inclusion dependencies of the classical relational theory [PDGV89, Sec. 3.6]), the general results are somewhat 
more limited. It does not appear that the techniques for the consistency-checking rules can be extended in a way that includes the behavior of such dependencies within a broad range of applications.

To remedy this limitation, at least partly, an investigation of decompositions of multi-relational schemata constrained by EGDs together with fanout-free unary inclusion dependencies (UINDs) is also conducted. The approach combines the general results for constraint-checking dependencies with a detailed investigation of how such UINDs decompose. The result is a positive one - both the EGDs and the UINDs have embedded covers within the views with no increase in complexity. It is also a potentially practical one, since UINDs are adequate to model referential integrity constraints, or foreign-key dependencies, which are commonly employed in real-world database systems. While these results, which are found in Section 6, are formally based upon the the framework presented earlier, the reader may nonetheless wish to look through this section to obtain an idea of the power of this approach within the relational setting.

The organization of this paper is as follows. Section 2 provides a concise overview of the key ideas of the constant-complement update strategy, including in particular recent work on uniqueness within order-based frameworks. In Section 3, the principles of $P F$-schemata and $P F$-views, the abstraction of these database concepts used in this paper, is elaborated and related to those used in earlier papers. In Section 4, the key ideas of the decomposition results of the earlier paper [Heg04a] are translated to this framework, and specialized notions essential to the measure of complexity are developed. In Section 5 , the general decomposition results, including in particular the embedding theorems for consistencychecking constraints, are established. Section 6 contains the detailed investigation within the context of multi-relational schemata constrained by both EGDs and UINDs. Section 7 offers some further directions and comparisons to the literature. Finally, Appendix A provides the details surrounding the axiomatizability issues of the example scheme $\mathbf{E}_{1}$ of 1.1 .

To close this section, some basic information on posets and relational notation which will be used throughout the paper is presented.

1.4 Posets, isomorphisms, and basic relational notation A partially ordered set (poset) is a pair $\mathbf{P}=(P, \leq)$ in which $P$ is a set and $\leq$ is a partial order on $P$. For $\mathbf{P}_{i}=\left(P_{i}, \leq\right)$ posets for $i=1$ and $i=2$, a morphism $f: \mathbf{P}_{1} \rightarrow \mathbf{P}_{2}$ is a function $f: P_{1} \rightarrow P_{2}$ with the property that for all $x, y \in P, x \leq y$ implies $f(x) \leq f(y)$. For any (not necessarily fi nite) set $X$, the poset $\mathscr{P}_{f}(X)=\left(\mathscr{P}_{f}(X), \subseteq\right)$ consists of all fi nite subsets of $X$, ordered by set inclusion. For additional background on posets, the reader is referred to [DP02].

As a general principle, following the standard mathematical convention [AHS90, 3.8], in all contexts an isomorphism will be taken to be a morphism which has both an left and a right inverse. Thus, if $f: X \rightarrow Y$ is an morphism in some context $C$, it is an isomorphism iff there is a morphism $g: X_{1} \rightarrow X_{2}$ with the properties that $g \circ f=\mathbf{1}_{X_{1}}$ and $f \circ g=\mathbf{1}_{X_{2}}$, with $\mathbf{1}_{X_{i}}$ the identity morphism on $X_{i}$. In the context of sets and functions, an isomorphism is a bijection, while in the context of posets, it is a bijection which both preserves and reflects order; i.e., $f$ is a poset isomorphism iff it is a bijection with the additional property that, for all $x, y \in P, f(x) \leq f(y)$ iff $x \leq y$.

Frequently, examples will be based upon the classical relational model, and the terminology and notation which has become standard over the past thirty years will be used without additional clarifi cation. For any questions, the reader is referred to [PDGV89] or [AHV95]. For this paper, it suffi ces to remark that given a universal relational schema $R[\mathbf{U}]$ on attribute set $\mathbf{U}$, and a subset $\mathbf{W} \subseteq \mathbf{U}, \Pi_{\mathbf{W}}$ will be used to denote the projective view of $R[\mathbf{U}]$ onto the attributes in $\mathbf{W}$, while $\pi_{\mathbf{W}}: R[\mathbf{U}] \rightarrow R[\mathbf{W}]$ will be used to denote the database mapping underlying this view. The notation $\operatorname{Dom}(A)$ will be used 
to denote the domain of $A$; i.e., the set of values associated with the attribute $A \in \mathbf{U}$.

Finally, the notation $\operatorname{Card}(A)$ will be used to denote the cardinality of the set $A$.

\section{An Overview of Previous Work}

While the results presented in this article do not deal with update strategies explicitly, they do address complexity issues which are cast within a context in which view updates are modelled and analyzed. Therefore, it is helpful to understand the underlying constant-complement update strategy. To provide the reader with this essential background, this section contains two summaries. 2.1 recaps the necessary aspects of closed update strategies within the relevant set-based framework. It provides ideas which originated with the work of Bancilhon and Spyratos of [BS81], although it is recast within the formalism of [Heg02] [Heg04a]. 2.2 sketches the key ideas developed in [Heg02] [Heg04a] which are necessary to extend the set-based ideas to the order-based context. These summaries include only the notions which are essential to the understanding of the current paper; the reader is referred to the aforementioned references for details and further clarifi cation. Since [Heg04a] is an expanded and corrected version of [Heg02], for the most part, only references to [Heg04a] will be given, even in the case that both papers contain the relevant material.

2.1 The classical results in the set-based framework In the original work of Bancilhon and Spyratos [BS81], a database schema $\mathbf{D}$ is just a set. To maintain consistency with the more structured frameworks to be introduced shortly, this set will be denoted $\operatorname{LDB}(\mathbf{D})$ and called the legal databases of $\mathbf{D}$. Thus, a database schema is modelled by its instances alone; constraints, schema structure, and the like are not represented explicitly. A morphism $f: \mathbf{D}_{1} \rightarrow \mathbf{D}_{2}$ of database schemata is a function $f: \operatorname{LDB}\left(\mathbf{D}_{1}\right) \rightarrow \operatorname{LDB}\left(\mathbf{D}_{2}\right)$.

A view of the schema $\mathbf{D}$ is a pair $\Gamma=(\mathbf{V}, \gamma)$ in which $\mathbf{V}$ is a schema and $\gamma: \mathbf{D} \rightarrow \mathbf{V}$ is a surjective database morphism. A morphism $f: \Gamma_{1} \rightarrow \Gamma_{2}$ of views $\Gamma_{1}=\left(\mathbf{V}_{1}, \gamma_{1}\right)$ and $\Gamma_{2}=\left(\mathbf{V}_{2}, \gamma_{2}\right)$ is a morphism $f: \mathbf{V}_{1} \rightarrow \mathbf{V}_{2}$ of schemata such that the diagram to the right commutes. The congruence of $\Gamma$ is the equivalence relation on $\operatorname{LDB}(\mathbf{D})$ defi ned by $\left(M_{1}, M_{2}\right) \in \operatorname{Congr}(\Gamma)$ iff $\gamma\left(M_{1}\right)=\gamma\left(M_{2}\right)$. It is easy to see that the views $\Gamma_{1}=\left(\mathbf{V}_{1}, \gamma_{1}\right)$ and $\Gamma_{2}=$ $\left(\mathbf{V}_{2}, \gamma_{2}\right)$ of $\mathbf{D}$ are isomorphic iff $\operatorname{Congr}\left(\Gamma_{1}\right)=\operatorname{Congr}\left(\Gamma_{2}\right)$.

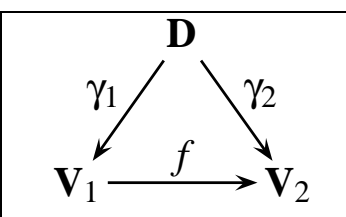

Figure 1: View morphism

A pair $\left\{\Gamma_{1}=\left(\mathbf{V}_{1}, \gamma_{1}\right), \Gamma_{2}=\left(\mathbf{V}_{2}, \gamma_{2}\right)\right\}$ of views of the schema $\mathbf{D}$ is called a subdirect complementary pair if it defi nes a lossless decomposition of $\mathbf{D}$. More precisely, the product $\Gamma_{1} \times \Gamma_{2}=$ $\left(\mathbf{V}_{1 \gamma_{1}} \otimes_{\gamma_{2}} \mathbf{V}_{2}, \gamma_{1} \otimes \gamma_{2}\right)$ has $\operatorname{LDB}\left(\mathbf{V}_{1 \gamma_{1}} \otimes_{\gamma_{2}} \mathbf{V}_{2}\right)=\left\{\left(\gamma_{1}(M), \gamma_{2}(M)\right) \mid M \in \operatorname{LDB}(\mathbf{D})\right\}$. The decomposition morphism $\gamma_{1} \otimes \gamma_{2}: \mathbf{D} \rightarrow \mathbf{V}_{1 \gamma_{1}} \otimes_{\gamma_{2}} \mathbf{V}_{2}$ is given on elements by $M \mapsto\left(\gamma_{1}(M), \gamma_{2}(M)\right)$. The set $\left\{\Gamma_{1}, \Gamma_{2}\right\}$ of views forms a subdirect complementary pair, and $\Gamma_{1}$ and $\Gamma_{2}$ are called subdirect complements of one another, just in case $\gamma_{1} \otimes \gamma_{2}$ is a bijection. In other words, $\left\{\Gamma_{1}, \Gamma_{2}\right\}$ is a subdirect complementary pair precisely in the case that the state of the schema $\mathbf{D}$ is recoverable from the combined states of $\mathbf{V}_{1}$ and $\mathbf{V}_{2}$.

To defi ne an update strategy on a view, the way in which such updates are reflected back to the main schema must be specifi ed. In a constant complement update strategy, as fi rst presented in [BS81], a (subdirect) complement is held constant. Thus, to update $\Gamma_{1}$ from $N_{1}$ to $N_{2}$ in the above context, the state of the schema $\mathbf{V}_{2}$ of the complement $\Gamma_{2}$ is held fi xed. Since the decomposition 
morphism is injective, it is easy to see that there can be only one way to translate an update in such a context.

A fundamental condition in the study of view updates,as reported in [Heg02], [Heg04a], and [Heg04b], is that the update strategies should be closed. The formal defi nition of an update strategy, and the list of conditions which render it closed, are quite technical and are not needed to understand the work reported here. The reader is therefore referred to [Heg04a, 3.1] for the complete, formal specifi cation. For this work, it suffi ces to understand the basic idea that a closed update strategy has the property that whether or not a given update is allowed does not depend upon the particular state of the complement. Thus, if a given update from $N_{1} \in \operatorname{LDB}\left(\mathbf{V}_{1}\right)$ to $N_{1}^{\prime} \in \operatorname{LDB}\left(\mathbf{V}_{1}\right)$ is allowed for some state $N_{2} \in \operatorname{LDB}\left(\mathbf{V}_{2}\right)$ of the complement, then it must be allowed for all states $N_{2}^{\prime} \in \operatorname{LDB}\left(\mathbf{V}_{2}\right)$ with the property that $\left(N_{1}, N_{2}^{\prime}\right) \in \operatorname{LDB}\left(\mathbf{V}_{1 \gamma_{1}} \otimes_{\gamma_{2}} \mathbf{V}_{2}\right)$.

Although every closed update strategy is defi nable via constant complement, not every subdirect complement of a view $\Gamma_{1}$ gives rise to closed update strategy. The property that a constant-complement update strategy be closed was termed $\Gamma_{2}$-translatability in [BS81, Def. 5.1]. However, it was not characterized explicitly until [Heg90a, 2.10], in which it was shown that $\Gamma_{2^{-}}$ translatability is characterized precisely by the property that the congruences of the views commute. Formally, the set $\left\{\Gamma_{1}, \Gamma_{2}\right\}$ of views of $\mathbf{D}$ is called a fully commut-

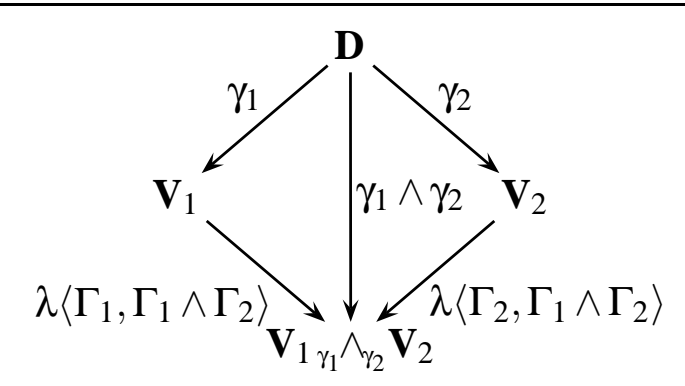

Figure 2: Relative views ing pair if Congr $\left(\Gamma_{1}\right) \circ \operatorname{Congr}\left(\Gamma_{2}\right)=\operatorname{Congr}\left(\Gamma_{2}\right) \circ \operatorname{Congr}\left(\Gamma_{1}\right)$, with "०" denoting ordinary composition of binary relations; i.e., $\left(M_{1}, M_{2}\right) \in \operatorname{Congr}\left(\Gamma_{1}\right) \circ \operatorname{Congr}\left(\Gamma_{2}\right)$ iff there is an $M_{3} \in \operatorname{LDB}(\mathbf{D})$ such that $\left(M_{1}, M_{3}\right) \in \operatorname{Congr}\left(\Gamma_{1}\right)$ and $\left(M_{3}, M_{2}\right) \in \operatorname{Congr}\left(\Gamma_{2}\right)$. A subdirect complementary pair $\left\{\Gamma_{1}, \Gamma_{2}\right\}$ which is fully commuting is called a meet-complementary pair, and $\Gamma_{1}$ and $\Gamma_{2}$ are called meet complements of one another. In this case, $\operatorname{Congr}\left(\Gamma_{1}\right) \circ \operatorname{Congr}\left(\Gamma_{2}\right)$ is also an equivalence relation on $\operatorname{LDB}(\mathbf{D})$, and so it is possible to defi ne (up to isomorphism) the view $\Gamma_{1} \wedge \Gamma_{2}=\left(\mathbf{V}_{1 \gamma_{1}} \wedge_{\gamma_{2}} \mathbf{V}_{2}, \gamma_{1} \wedge \gamma_{2}\right)$ with Congr $\left(\Gamma_{1} \wedge \Gamma_{2}\right)=\operatorname{Congr}\left(\Gamma_{1}\right) \circ \operatorname{Congr}\left(\Gamma_{2}\right)$, called the meet of $\left\{\Gamma_{1}, \Gamma_{2}\right\}$. It is, in effect, a wellbehaved greatest lower bound of $\left\{\Gamma_{1}, \Gamma_{2}\right\}$. The situation is summed up in Fig. 2 above. Note that $\mathbf{V}_{1 \gamma_{1}} \wedge_{\gamma_{2}} \mathbf{V}_{2}$ is (the schema of) a view not only of $\mathbf{D}$, but of $\mathbf{V}_{1}$ and $\mathbf{V}_{2}$ as well. The mappings $\lambda\left\langle\Gamma_{i}, \Gamma_{1} \wedge \Gamma_{2}\right\rangle: \mathbf{V}_{i} \rightarrow \mathbf{V}_{1 \gamma_{1}} \wedge_{\gamma_{2}} \mathbf{V}_{2}$ for $i=1,2$ are the morphisms of the associate relative views (see [Heg04a, 2.8]; or 3.12 for a development within the special context of this paper). In the context of updates, if $\left\{\Gamma_{1}, \Gamma_{2}\right\}$ forms a meet-complementary pair, then an update to the schema of $\Gamma_{1}$ is allowed with constant complement $\Gamma_{2}$ iff that update keeps the state of the meet schema $\mathbf{V}_{1 \gamma_{1}} \wedge_{\gamma_{2}} \mathbf{V}_{2}$ constant. Since the meet schema may be regarded as a view of $\Gamma_{1}$, knowledge of the precise state of the view of $\Gamma_{2}$ is not necessary.

For a concrete example, identify $\mathbf{D}$ with the schema $\mathbf{E}_{2}$ of 1.2 and 1.3, identify $\Gamma_{1}$ with $\Pi_{A B C E}$, and

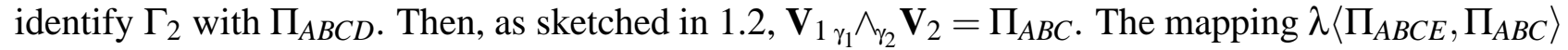
is the projection $\pi_{A B C}: S[A B C E] \rightarrow S[A B C]$. Similarly, $\lambda\left\langle\Pi_{A B C D}, \Pi_{A B C}\right\rangle$ is the projection $\pi_{A B C}$ : $S[A B C D] \rightarrow S[A B C]$. On the other hand, $\gamma_{1} \wedge \gamma_{2}$ is the projection $\pi_{A B C}: S[A B C D E] \rightarrow S[A B C]$. More generally, in the context of relational schemata and views defi ned by projection, a pair of views forms a meet-complementary pair iff the decomposition is both lossless and dependency preserving [Heg04a, 2.17]. In this case, the meet view is just the projection on the common columns. To obtain an example in which the views form a subdirect complementary pair but not a meet complementary pair, it suffi ces to consider an example which is lossless but not dependency preserving, as sketched 
in 1.3 .

In [Heg93], the connection between decompositions of database schemata and commuting congruences is investigated thoroughly.

2.2 The order-based framework Despite its simplicity and elegance, the set-based framework for closed update strategies has a substantial shortcoming; namely, the update strategy depends upon the choice of the complement. For example, let $\mathbf{F}_{0}$ be the relational schema with the single relation $R[A B C]$, constrained by the single FD $B \rightarrow C$, and let $\Pi_{A B}$ be the view defi ned by the projection mapping $\pi_{A B}$. Defi ne $\Pi_{B C}$ similarly. Since the pair $\left\{\Pi_{A B}, \Pi_{B C}\right\}$ forms a lossless and dependencypreserving decomposition of $\mathbf{F}_{0}$, it also forms a meet-complementary pair [Heg04a, 2.17]. Indeed, $\Pi_{B C}$ is the "natural" complement of $\Pi_{A B}$, and the one which yields the "obvious" strategy for reflecting updates to $\Pi_{A B}$ back to $\mathbf{F}_{0}$. However, as shown in [Heg04a, 1.3], it is possible to fi nd other complements of $\Pi_{A B}$ which have exactly the same meet, and so support exactly the same updates to $\Pi_{A B}$. Although these alternate complements are a bit pathological, the set-based theory outlined above in 2.1 does not prefer $\Pi_{B C}$ to them in any way.

To formalize this preference, additional structure must be incorporated into the model. Most database models incorporate some sort of order structure. In the relational model, the databases may be ordered via relation-by-relation inclusion. Furthermore, the common database mappings built from projection, selection, and join are all order preserving with respect to this natural order structure. In particular, while the views $\Pi_{A B}$ and $\Pi_{B C}$ are order mappings, the alternate views identifi ed in [Heg04a, 2.17] are not.

The theory developed in [Heg04a] provides a systematic extension to the results outlined in 2.1 above to the order-based setting. An order schema $\mathbf{D}$ is taken to be a poset $\left(\operatorname{LDB}(\mathbf{D}), \leq_{\mathbf{D}}\right)$. An order database mapping $f: \mathbf{D}_{1} \rightarrow \mathbf{D}_{2}$ is an order-preserving function; i.e., $M_{1} \leq_{\mathbf{D}_{1}} M_{2}$ implies $f\left(M_{1}\right) \leq_{\mathbf{D}_{2}}$ $f\left(M_{2}\right)$. An order view $\Gamma=(\mathbf{V}, \gamma)$ of $\mathbf{D}$ consists of an order schema $\mathbf{V}$ and an open surjection $\gamma$ : $\operatorname{LDB}(\mathbf{D}) \rightarrow \operatorname{LDB}(\mathbf{V})$; that is, a surjection which is order preserving and, in addition, which satisfi es the property that whenever $N_{1} \leq_{\mathbf{v}} N_{2}$, there are $M_{1}, M_{2} \in \operatorname{LDB}(\mathbf{D})$ with the properties that $M_{1} \leq_{\mathbf{D}} M_{2}$, $f\left(M_{1}\right)=N_{1}$, and $f\left(M_{2}\right)=N_{2}$. For the pair of order views $\left\{\Gamma_{1}, \Gamma_{2}\right\}$ to form a subdirect complementary pair in the order sense, the mapping $\gamma_{1} \otimes \gamma_{2}: \mathbf{D} \rightarrow \mathbf{V}_{1 \gamma_{1}} \otimes_{\gamma_{2}} \mathbf{V}_{2}$ must be an order isomorphism (i.e., an order database mapping which is an isomorphism), and not merely an order-preserving bijection. To obtain a closed update strategy in the oder-bases sense, the formal conditions which apply in the set-based framework must be augmented with special conditions which guarantee that the update strategy respects the order structure [Heg04a, 3.1]. Modulo these modifi cations, it is fair to say, at least in a general way, that [Heg04a] extends the classical set-based constant complement theory to the order-based setting. As a rich source of classical but important examples, all SPJR-mappings (Select, Project, Join, Rename) in the classical relational setting defi ne order views [Heg04a, 2.5]. The results of the current paper are cast within a more restrictive order-based context in which selection and projection, but not join, defi ne views.

In [Heg94], a theory of direct decomposition (i.e., situations in which the views are independent and so the meet is trivial) of order-based schemata is presented. 


\section{PF-Schemata and PF-Views}

The database schemata with order of [Heg04a] and summarized in 2.2 model only those databases which satisfy the constraints of the schema; those which fail to satisfy those constraints are simply not part of the formalism. In order to model the complexity of constraint checking, it is necessary to employ a formalism which includes all databases, including those which fail to satisfy those constraints. Furthermore, to have a basis for measuring the complexity, the extended formalism must provide some notion of size of a database.

In this section, the foundations of such a framework are presented. Although it has much in common with the earlier work [Heg04b], it differs in a very fundamental way. Specifi cally, in [Heg04b], the starting point was the so-called semantic schemata - those which include only the databases which satisfy the constraints of the schema. From the semantic schemata the more general syntactic schemata - those which embody all databases, regardless of whether or not they satisfy the constraints - were constructed, using a sort of "completion" operation. While this approach is effective, it is also somewhat limiting, in that it presupposes suffi cient structure on the semantic schemata and their morphisms to yield unique and well-defi ned completions. This requirement is, unfortunately, not always met in practical examples. In the approach taken here, the starting point is the syntactic schema, with the semantic properties imposed later. As will be seen, this allows a more general class of constraints to be modelled, since unique completions are no longer required.

3.1 CFA-schemata and morphisms In order to capture the complexity of checking constraint satisfaction, the formal notion database schema must contain two pieces of information which are not present in the general order-based schemata of [Heg04a]. First of all, the order schema model of [Heg04a] contains only the legal databases; the idea that there are also databases which do not satisfy the constraints is not represented. Second, there is no measure of database size, without which there is no possibility of measuring complexity. To address these issues, in [Heg04b] the notions of CFAschema and CFA-morphism were introduced. In the current paper, these notions have been replaced by the more flexible PF-schema and PF-morphism, respectively. The purpose of this subsection is to tie the "CFA" notions to their newer "PF" counterparts, and may safely be skipped by the reader not interested in the evolution of these ideas.

In $[\mathrm{Heg} 04 \mathrm{~b}]$, the set of all databases, legal or not, was modelled by $\mathscr{P}_{f}(X)$ for some (not necessarily fi nite) set $X$, while the legal databases were modelled by a special type of sub-poset of $\mathscr{P}_{f}(X)$, called a CFA-poset. Clearly, choosing the databases to be fi nite sets gives a simple measure of size - the cardinality of the set. Furthermore, taking the legal databases to be a subset of the set of all databases is completely natural and obvious. In the context of the classical relational model, think of $X$ as the set of all possible tuples of the relation(s).

Formally, a $C F A$-poset over $X$ is a sub-poset of $\mathscr{P}_{f}(X)$ which contains all singleton sets, as well as the empty set. This choice was made for the model of a database schema because it facilitated the definition of database morphisms within this context. Specifi cally, if $\mathbf{P}=(P, \subseteq)$ is a CFA-poset, defi ne $\operatorname{Atoms}(\mathbf{P})=\{\{x\} \mid x \in \bigcup P\}, \operatorname{ExtAtoms}(\mathbf{P})=\operatorname{Atoms}(\mathbf{P}) \cup\{\emptyset\}$, and Foundation $(\mathbf{P})=\bigcup \operatorname{Atoms}(\mathbf{P})$. For any $M \in P$, the basis of $M$ is $\operatorname{Basis}_{\mathbf{P}}(M)=\{\{x\} \mid x \in M\}$. A CFA-schema is just a CFAposet. CFA-morphisms were then modelled as basis-preserving mappings; that is, if $\mathbf{P}=(P, \subseteq)$ and $\mathbf{Q}=(Q, \subseteq)$ are CFA-posets, then a CFA-morphism is a function $f: P \rightarrow Q$ with the property that, for all $M \in P,\left\{f(a) \mid a \in \operatorname{Basis}_{\mathbf{P}}(M)\right\} \backslash\{\emptyset\}=\operatorname{Basis}_{\mathbf{Q}}(f(M)) .^{2}$ In other words, a CFA-morphism

\footnotetext{
${ }^{2}$ Unfortunately, the defi nition given in $[\mathrm{Heg} 04 \mathrm{~b}]$ is incorrect. The condition that $\{f(a) \mid a \in \operatorname{Basis}(M)\} \backslash\{\emptyset\}=$
} 
is completely defi ned by its action on singletons, and the image of each singleton must itself be a singleton or the empty set. The advantage of this defi nition is that it embodies the syntactic morphism as well as the syntactic one. More precisely, a morphism $f: \mathbf{P} \rightarrow \mathbf{Q}$ may be extended to $\bar{f}: \mathscr{P}_{f}($ Foundation $(\mathbf{P})) \rightarrow \mathscr{P}_{f}($ Foundation $(\mathbf{Q}))$ by defi $\operatorname{ning} \bar{f}(M)=\bigcup\{f(\{x\}) \mid x \in M\}$. This $\bar{f}$ is the syntactic extension of $f$ to all databases, legal or not. (As a concrete example, think of $f$ as specifying a projection on the legal relations of a universal schema. $\bar{f}$ extends $f$ to all relations, regardless of whether or not they satisfy the constraints.)

3.2 PF-schemata In a CFA-schema, all singletons, as well as the empty set, are legal databases. Translated to a universal relational schema, this means that the empty relation and all relations containing just one tuple are legal. If all constraints are typed and universal (e.g., equality generating dependencies and full implicational dependencies), this condition is automatically satisfi ed. However, relational schemata governed by dependencies involving existential quantifi cation, such as embedded join dependencies, inclusion dependencies, and even foreign-key dependencies, will not have this property. Since the scope of the work here is to include specifi cally such flavors of constraints, the model of CFA-schema must be augmented. The trick is to include two pieces of information with each schema. The fi rst provides the equivalent of the foundation of a CFA-schema, and the second gives the legal databases, taken as a subset of the fi nite powerset over that foundation. While this may seem to be a bit heavy handed, remember that this is a mathematical model, not a blueprint for implementation. It is the most convenient means of recapturing all of the information necessary.

(a) A PF-schema is an ordered pair $\mathbf{D}=(\operatorname{SynFnd}(\mathbf{D}), \operatorname{LDB}(\mathbf{D}))$, in which SynFnd $(\mathbf{D})$ is a nonempty set (not necessarily fi nite), with $\operatorname{LDB}(\mathbf{D}) \subseteq \mathscr{P}_{f}(\operatorname{Syn} F n d(\mathbf{D}))$. (The prefi x $P F$ - is derived from the notation $\mathscr{P}_{f}(-)$.) The set SynFnd(D) is called the syntactic foundation of $\mathbf{D}$, and $\operatorname{LBB}(\mathbf{D})$ is the set of legal databases of $\mathbf{D}$.

Note that there is no requirement that each $x \in \operatorname{SynFnd}(\mathbf{D})$ occur in some $M \in \operatorname{LDB}(\mathbf{D})$, much less that $\{x\} \in \operatorname{LDB}(\mathbf{D})$ and $\emptyset \in \operatorname{LDB}(\mathbf{D})$ hold, so that the limitations of CFA-posets do not apply. In the context of a classical one-relation schema, think of SynFnd $(\mathbf{D})$ as the set of tuples which the relation may contain, and $\operatorname{LDB}(\mathbf{D})$ as the set of relations over those tuples which satisfy the constraints of the schema.

For the rest of this subsection, assume that $\mathbf{D}$ is a PF-schema. There is not one but three order relations associated with $\mathbf{D}$.

(b) The poset of legal databases of $\mathbf{D}$ is just $\operatorname{LDBPoset}(\mathbf{D})=(\operatorname{LDB}(\mathbf{D}), \subseteq)$.

Note that LDBPoset $(\mathbf{D})$ is a database schema with order, in the sense of [Heg04a], although it is not necessarily a CFA-schema in the sense of [Heg04b], since it need not contain all singletons and the empty set. It is the syntactic foundation poset which contains the representation of the latter.

(c) The extended syntactic foundation of $\mathbf{D}$ is $\operatorname{ExtSynFnd}(\mathbf{D})=\operatorname{SynFnd}(\mathbf{D}) \cup\left\{\eta_{\mathbf{D}}\right\}$, in which $\eta_{\mathbf{D}}$ is a special symbol, called the null element of $\mathbf{D}$, which does not occur in SynFnd(D).

(d) The syntactic foundation poset of $\mathbf{D}$ is $\operatorname{SFPoset}(\mathbf{D})=\left(\operatorname{ExtSynFnd}(\mathbf{D}), \leq_{\mathrm{SF}(\mathbf{D})}\right)$. The ordering $\leq_{\mathrm{SF}(\mathbf{D})}$ is defi ned to be that in which $\eta_{\mathbf{D}} \leq_{\mathrm{SF}(\mathbf{D})} x$ for all $x \in \operatorname{SynFnd}(\mathbf{D})$, but is otherwise flat.

$\operatorname{Basis}_{\mathbf{Q}}(f(M))$, which is the one intended, is expressed incorrectly as $\bigcup\{f(a) \mid a \in \operatorname{Basis} \mathbf{P}(M)\}=\operatorname{Basis}_{\mathbf{Q}}(f(M))$. 
In other words, $x \leq_{\mathrm{SF}(\mathbf{D})} y$ holds iff $x=\eta_{\mathbf{D}}$ or else $x=y$. SFPoset $(\mathbf{D})$ is trivially a database schema with order. Think of $\eta_{\mathbf{D}}$ as a special marker which represents the empty set as a database. Its rôle will become apparent later, in 3.4 for the defi nition of a PF-morphism and in 3.9 for the modelling of an atomic equivalence relation.

The third poset is the disjoint union of the fi rst two. Its use is purely technical; it makes it possible to consider a PF-schema as an order schema in the sense of [Heg04a].

(e) Defi ne ExtSynFnd $(\mathbf{D}) \boxplus \operatorname{LDB}(\mathbf{D})$ to be the disjoint union of these two sets, and defi ne the order $\leq_{\boxplus(\mathbf{D})}$ on $\operatorname{ExtSynFnd}(\mathbf{D}) \boxplus \operatorname{LDB}(\mathbf{D})$ to be the (disjoint) union of $\subseteq$ on $\operatorname{LDB}(\mathbf{D})$ and $\leq_{\mathrm{SF}(\mathbf{D})}$ on $\operatorname{ExtSynFnd(D).~}$

(f) Defi ne the combined poset of $\mathbf{D}$ to be $\operatorname{DJPoset}(\mathbf{D})=\left(\operatorname{ExtSynFnd}(\mathbf{D}) \boxplus \operatorname{LDB}(\mathbf{D}), S_{\boxplus(\mathbf{D})}\right)$.

$\operatorname{DJPoset}(\mathbf{D})=\left(\operatorname{ExtSynFnd}(\mathbf{D}) \boxplus \operatorname{LDB}(\mathbf{D}), \leq_{\boxplus(\mathbf{D})}\right)$ is clearly a database schema with order, in the sense of [Heg04a]. It is not necessarily a CFA-poset, although it embodies the information necessary to extend LDBPoset(D) uniquely to a CFA-poset.

The syntactic schema of $\mathbf{D}$, defi ned below, is constructed directly from the extended syntactic foundation; its elements represent all databases, legal or not. Note that this schema is itself a PFschema, in the sense of (a) above, and all properties associated with PF-schemata apply to it as well.

(g) For $\mathbf{D}=(\operatorname{SynFnd}(\mathbf{D}), \operatorname{LDB}(\mathbf{D}))$, defi ne $\overline{\mathbf{D}}=(\operatorname{SynFnd}(\mathbf{D}), \operatorname{DB}(\mathbf{D}))$ to have $\mathrm{DB}(\mathbf{D})=$ $\mathscr{P}_{f}(\operatorname{SynFnd}(\mathbf{D})) . \overline{\mathbf{D}}$ is called the syntactic schema over which $\mathbf{D}$ is taken.

To avoid confusion, when $\emptyset$ is considered as an element of $\operatorname{LDB}(\mathbf{D})$ or $\operatorname{DB}(\mathbf{D})$, it will sometimes be written as $\perp_{\mathbf{D}}$ and $\perp_{\overline{\mathbf{D}}}$, respectively.

Finally, for a database $M$, it is occasionally advantageous to be able to augment $M$ with the null element for $\mathbf{D}$. This augmentation has no special semantics, but is useful in establishing certain results in which the empty set behaves like an atom.

(h) For $M \in \mathrm{DB}(\mathbf{D})$, Defi ne $\operatorname{NullExt}(M)=M \cup\left\{\eta_{\mathbf{p}}\right\}$.

3.3 Example - PF-schema Let $\mathbf{E}_{4}$ be the two-relation schema consisting of $R_{1}[A B C]$ and $R_{2}[D E F]$. Defi ne $\mathcal{F}_{4}=\{A \rightarrow B, D \rightarrow E F\}$, and assume that these constraints hold on the schema. SynFnd $\left(\mathbf{E}_{4}\right)$ consists of all tuples over these relations; that is, it consists of all tuples on $\operatorname{Dom}(A) \times$ $\operatorname{Dom}(B) \times \operatorname{Dom}(C)$, together with all tuples on $\operatorname{Dom}(D) \times \operatorname{Dom}(E) \times \operatorname{Dom}(F)$. These tuples must somehow be tagged, so that it is known to which relation they belong. In this example, domain elements will be represented by (possibly subscripted) lower case letters which match the domain name; so, for example, $\left(a_{1}, b_{1}, c_{1}\right)$ is associated with $R_{1}$, while $\left(d_{1}, e_{1}, f_{1}\right)$ is associated with $R_{2}$. Thus, the tagging is implicit in the naming convention.

The set SynFnd $\left(\mathbf{E}_{4}\right)$ is then just the set of all such tuples. The extended syntactic foundation ExtSynFnd $\left(\mathbf{E}_{4}\right)$ adds one additional element, which would be called $\eta_{\mathbf{E}_{4}}$ in the notation of 3.2. The ordering on the syntactic foundation poset SFPoset $\left(\mathbf{E}_{4}\right)$ has $\eta_{\mathbf{E}_{4}}$ at the bottom, with all tuples otherwise incomparable.

The set $\mathrm{DB}\left(\mathbf{E}_{4}\right)$ is the fi nite powerset of $\operatorname{SynFnd}\left(\mathbf{E}_{4}\right)$; that is, the set of all fi nite subsets of tuples, while $\operatorname{LDB}\left(\mathbf{E}_{4}\right)$ is the subset of $\operatorname{DB}\left(\mathbf{E}_{4}\right)$ consisting of sets which satisfy the constraints in $\mathcal{F}_{4}$. Note that in this formalism it is necessary to collect the set of all tuples of a database instance into a single set, rather than the more common practice of having a separate set of tuples for each relation. Since 
the tuples are tagged, this amounts to an inessential syntactic variation which has no impact upon the underlying theory.

3.4 PF-morphisms In the same manner that PF-schemata generalize CFA-schemata, PF-morphisms generalize CFA-morphisms. In the PF-context, it is no longer possible to stipulate the basis-preserving property directly on the mappings between legal databases, because the basis itself may not be embedded in those states. Rather, the starting point for a PF-morphism is a mapping between the extended syntactic foundations, which replace the bases of the CFA-context. The mapping between legal databases is then defi ned as an extension of the mapping between the foundations.

To begin, it is necessary to formalize the notion of an elementary mapping, as well as its extensions. For the remainder of this subsection, let $\mathbf{D}_{1}=\left(\operatorname{SynFnd}\left(\mathbf{D}_{1}\right), \operatorname{LDB}\left(\mathbf{D}_{1}\right)\right)$ and $\mathbf{D}_{2}=\left(\operatorname{SynFnd}\left(\mathbf{D}_{2}\right), \operatorname{LDB}\left(\mathbf{D}_{2}\right)\right)$ be PF-schemata.

(a) An elementary mapping is a function $e: \operatorname{ExtSynFnd}\left(\mathbf{D}_{1}\right) \rightarrow \operatorname{ExtSynFnd}\left(\mathbf{D}_{2}\right)$ with the property that $e\left(\eta_{\mathbf{D}_{1}}\right)=\eta_{\mathbf{D}_{2}}$.

(b) The full extension of the elementary mapping $e$ is the function $e^{+}: \mathrm{DB}\left(\mathbf{D}_{1}\right) \rightarrow \mathrm{DB}\left(\mathbf{D}_{2}\right)$ given on elements by $M \mapsto\{e(x) \mid x \in M\} \backslash\left\{\eta_{\mathbf{D}_{2}}\right\}$.

A few remarks are appropriate at this point, regarding how null elements are involved in the above defi nitions. In the defi nition of CFA-morphism in 3.1, a basis preserving morphism was required to map atoms to extended atoms; that is, each atom was mapped either to another atom or else to the null set. However, the members of $\operatorname{SynFnd}\left(\mathbf{D}_{i}\right)$ are foundation elements, and not atoms (i.e., they are of the form $t$, not $\{t\}$ ). Therefore, it is necessary to employ the special marker $\eta_{\mathbf{D}_{i}}$ as a sort of surrogate for $\emptyset=\{\}$ with one set of brackets stripped away. More concretely, observe that $e: t \mapsto \eta_{\mathbf{D}_{2}}$ means that $e^{+}:\{t\} \mapsto \emptyset=\perp_{\mathbf{D}_{2}}$. Note also that the null elements are always stripped away in the full extension, since that function deals with databases, of which a null element is never a member.

It may also be remarked that an elementary mapping could have been defi ned to be of the form $e: \operatorname{SynFnd}\left(\mathbf{D}_{1}\right) \rightarrow \operatorname{ExtSynFnd}\left(\mathbf{D}_{2}\right)$, since null elements are not needed in the domain. However, this would have complicated the defi nition of morphism composition somewhat. Including $\eta_{\mathbf{D}_{1}}$ in the domain of $e$ is harmless enough, since it is always mapped to $\eta_{\mathbf{D}_{2}}$.

Next, the details of extending an elementary mapping to mappings between databases are elaborated.

(c) The elementary mapping $e$ is semantically stable if $e^{+}\left(\operatorname{LDB}\left(\mathbf{D}_{1}\right)\right) \subseteq \operatorname{LDB}\left(\mathbf{D}_{2}\right)$; i.e., for each $M \in \operatorname{LDB}\left(\mathbf{D}_{1}\right), e^{+}(M) \in \operatorname{LDB}\left(\mathbf{D}_{2}\right)$.

(d) Formally, a PF-morphism $f: \mathbf{D}_{1} \rightarrow \mathbf{D}_{2}$ is a triple $\left(f^{\sharp}, f^{\natural}, f^{\boxplus}\right)$ in which

(i) $f^{\sharp}: \operatorname{ExtSynFnd}\left(\mathbf{D}_{1}\right) \rightarrow \operatorname{ExtSynFnd}\left(\mathbf{D}_{2}\right)$ is a semantically stable elementary mapping;

(ii) $f^{b}: \operatorname{LDB}\left(\mathbf{D}_{1}\right) \rightarrow \operatorname{LDB}\left(\mathbf{D}_{2}\right)$ with $f^{\dagger}(M)=\left(f^{\sharp}\right)^{+}(M)$ for all $M \in \operatorname{LDB}(\mathbf{D})$.

(iii) $f^{\boxplus}: \operatorname{ExtSynFnd}\left(\mathbf{D}_{1}\right) \boxplus \operatorname{LDB}\left(\mathbf{D}_{1}\right) \rightarrow \operatorname{ExtSynFnd}\left(\mathbf{D}_{2}\right) \boxplus \operatorname{LDB}\left(\mathbf{D}_{2}\right)$ given by $f^{\boxplus}(x)=f^{\sharp}(x)$ if $x \in \operatorname{SynFnd}\left(\mathbf{D}_{1}\right)$, and $f^{\boxplus}(x)=f^{b}(x)$ if $x \in \operatorname{LDB}\left(\mathbf{D}_{1}\right)$.

This defi nition may seem to be needlessly complex, since $f$ is completely determined by $f^{\sharp}$, and $f^{\boxplus}$ is obtained by combining $f^{\sharp}$ and $f^{b}$. However, a notation is needed for all three components in any case, and including them explicitly in the defi nition causes no harm, while making the defi nition of morphism composition more concrete. 
Composition of morphisms is defi ned in the obvious way; that is, $f \circ g$ is given by the triple $\left(f^{\sharp} \circ g^{\sharp}, f^{b} \circ g^{b}, f^{\boxplus} \circ g^{\boxplus}\right)$.

It is clear that all three of $f^{\sharp}: \operatorname{SFPoset}\left(\mathbf{D}_{1}\right) \rightarrow \operatorname{SFPoset}\left(\mathbf{D}_{2}\right), f^{b}: \operatorname{LDBPoset}\left(\mathbf{D}_{1}\right) \rightarrow \operatorname{LDBPoset}\left(\mathbf{D}_{2}\right)$, and $f^{\boxplus}: \operatorname{DJPoset}\left(\mathbf{D}_{1}\right) \rightarrow$ DJPoset $\left(\mathbf{D}_{2}\right)$ may be viewed as poset morphisms. $f^{\boxplus}$ embodies the ideas of CFA-morphism, although it is not formally one itself.

The notions of a surjective morphism and an open morphism must be defi ned carefully here, since they focus on $\operatorname{LDB}(\mathbf{D})$. The defi nition of isomorphism is standard [AHS90, 3.8].

(e) The PF-morphism $f$ will be called surjective precisely in the case that both $f^{\sharp}: \operatorname{ExtSynFnd}\left(\mathbf{D}_{1}\right) \rightarrow$ $\operatorname{ExtSynFnd}\left(\mathbf{D}_{2}\right)$ and $f^{b}: \operatorname{LDB}\left(\mathbf{D}_{1}\right) \rightarrow \operatorname{LDB}\left(\mathbf{D}_{2}\right)$ are surjective. (Note that this is not equivalent to the surjectivity of $f^{\sharp}$ alone.)

(f) The PF-morphism $f$ is open if for any $N_{1}, N_{2} \in \operatorname{LDB}\left(\mathbf{D}_{2}\right)$ with $N_{1} \subseteq N_{2}$, there are $M_{1}, M_{2} \in$ $\operatorname{LDB}\left(\mathbf{D}_{1}\right)$ with $M_{1} \subseteq M_{2}$ and $f^{b}\left(M_{1}\right)=N_{1}, f^{\prime}\left(M_{2}\right)=N_{2}$.

(g) The PF-morphism $f$ is a $P F$-isomorphism if there is a morphism $g: \mathbf{D}_{2} \rightarrow \mathbf{D}_{1}$ with the property that $g \circ f$ is the identity on $\mathbf{D}_{1}$ and $f \circ g$ is the identity on $\mathbf{D}_{2}$.

Finally, it is useful to have a special notation for the PF-morphism between the syntactic schemata derived from $\mathbf{D}_{1}$ and $\mathbf{D}_{2}$.

(h) Defi ne the PF-morphism $\bar{f}: \overline{\mathbf{D}}_{1} \rightarrow \overline{\mathbf{D}}_{2}$ as $\left(\bar{f}^{\sharp}, \bar{f}^{\prime}, \bar{f}^{\boxplus}\right)$, with $\bar{f}^{\sharp}=\bar{f}, \bar{f}^{\natural}=f^{\sharp+}$, and $\bar{f}^{\boxplus}$ given by $\bar{f}^{\boxplus}(x)=f^{\sharp}(x)$ if $x \in \operatorname{SynFnd}\left(\mathbf{D}_{1}\right)$, and $\bar{f}^{\boxplus}(x)=\bar{f}^{\prime}(x)$ if $x \in \operatorname{DB}\left(\mathbf{D}_{1}\right)$.

3.5 Example - PF-morphisms The example builds upon the context introduced in 3.3. Let $\mathbf{W}_{1_{A B}}$ be the schema whose sole relation is $R_{3}[A B]$, constrained by the FD $A \rightarrow B$. Defi ne the elementary mapping $h_{4}: \operatorname{ExtSynFnd}\left(\mathbf{E}_{4}\right) \rightarrow \operatorname{ExtSynFnd}\left(\mathbf{W}_{1_{A B}}\right)$ by $(a, b, c) \mapsto(a, b)$ if $(a, b, c) \in \operatorname{Dom}(A) \times$ $\operatorname{Dom}(B) \times \operatorname{Dom}(C),(d, e, f) \mapsto \eta_{\mathbf{w}_{1_{A B}}}$ if $(d, e, f) \in \operatorname{Dom}(D) \times \operatorname{Dom}(E) \times \operatorname{Dom}(F)$, and $\eta_{\mathbf{E}_{4}} \mapsto \eta_{\mathrm{w}_{1_{A B}}}$.

The full extension $h_{4}{ }^{+}: \mathrm{DB}\left(\mathbf{E}_{4}\right) \rightarrow \mathrm{DB}\left(\mathbf{W}_{1_{A B}}\right)$ is the morphism which projects each tuple of the instance of $R_{1}[A B C]$ onto its $A B$ projection, and ignores all tuples in the instance of $R_{2}[D E F]$, while $h_{4}{ }^{b}: \operatorname{LDB}\left(\mathbf{E}_{4}\right) \rightarrow \operatorname{LDB}\left(\mathbf{W}_{1_{A B}}\right)$ exhibits the same behavior on the instances satisfying $\mathcal{F}_{4}$. Thus, if $M=$ $\left\{\left(a_{1}, b_{1}, c_{1}\right),\left(a_{2}, b_{2}, c_{2}\right),\left(d_{1}, e_{1}, f_{1}\right),\left(d_{2}, e_{2}, f_{2}\right)\right\}$, then $\omega_{1_{A B}}(M)=\left\{\left(a_{1}, b_{1}\right),\left(a_{2}, b_{2}\right)\right\}$. The morphism $h_{4}$ is clearly semantically stable; however, if the constraint $A \rightarrow B$ were dropped on $\mathbf{E}_{4}$, with that same constraint retained on $R_{3}[A B]$, then semantic stability would no longer hold.

It is important to note in particular the need for the null elements $\eta_{\mathrm{w}_{1}{ }_{A B}}$. Without it, it would not be possible to specify the behavior of $\omega_{1_{A B}}$ on an element-by-element basis, since it would then be impossible to specify the image of tuples in the instance of $R_{2}[D E F]$.

3.6 The limitations of PF-morphisms In a PF-morphism, the mapping is defi ned element by element. Thus, in the context of the classical relational model, a PF-morphism can represent operations such as projection, selection, and renaming. However, join cannot be so represented, since the join operation involves the combination of two tuples. This may seem to be a rather severe limitation, but it is necessary to obtain the results developed in this work. Element-by-element operators, such as projection and restriction, do not generate any constraints themselves; rather, they simply pass along information about the constraints which already exist. On the other hand, an operation such as join imposes a constraint (a join dependency) itself on the image, a constraint which may not exist on the domain. Thus, a situation in which a single element in the view schema is constructed from several 
elements in the main schema complicates matters enormously. The view mapping may itself impose constraints, so that constraints now come from two sources, the other being the implied constraints from the main schema. It seems most prudent here to begin with PF-morphisms, and to look for extensions only after the theory for these element-by-element mappings is understood better.

3.7 PF-views The abstract defi nition of a PF-view parallels closely that of an order view [Heg04a, 2.3] and a CFA-view [Heg04b, 3.2]. Specifi cally, let (SynFnd(D), LDB(D)) be a PF-schema.

(a) A PF-view of $\mathbf{D}$ is a pair $\Gamma=(\mathbf{V}, \gamma)$ in which $\mathbf{V}=(\operatorname{SynFnd}(\mathbf{V}), \operatorname{LDB}(\mathbf{V}))$ is a PF-schema and $\gamma: \mathbf{D} \rightarrow \mathbf{V}$ is an open surjective PF-morphism.

Note that open and surjective have the meanings assigned in 3.4(e)-(f) above. Furthermore, it is easy to see that $\Gamma_{1}=\left(\mathbf{V}_{1}, \gamma_{1}\right)$ and $\Gamma_{2}=\left(\mathbf{V}_{2}, \gamma_{2}\right)$ of $\mathbf{D}$ are isomorphic if there is a PF-isomorphism $f: \mathbf{V}_{1} \rightarrow \mathbf{V}_{2}$ with the property that $f \circ \gamma_{1}=\gamma_{2}$. Indeed, if $f$ is a PF-isomorphism, then $f^{-1} \circ \gamma_{2}=$ $f^{-1} \circ\left(f \circ \gamma_{1}\right)=\left(f^{-1} \circ f\right) \circ \gamma_{1}=\gamma_{1}$.

In the context of updates, it is important to have available the associated syntactic view, which is defi ned as follows.

(b) The extension of $\Gamma$ to $\overline{\mathbf{D}}$ is $\bar{\Gamma}=(\overline{\mathbf{V}}, \bar{\gamma})$.

It is straightforward to verify that $\bar{\Gamma}$ is a PF-view of $\overline{\mathbf{D}}$.

3.8 Congruences on a PF-schema The defi nition of closed update strategy is made within the context of meet-complementary views, and the defi nition of the latter is based centrally upon the congruences of those views. It is therefore critical to classify these congruences and identify their rôles in the construction of complementary and meet-complementary views. Just as a morphism has three functional components, so too does a view have three congruences. In addition, the congruence of the syntactic extension $\bar{f}$ is also identifi ed explicitly, since it is used in subsequent proofs.

Let $\mathbf{D}_{1}=\left(\operatorname{SynFnd}\left(\mathbf{D}_{1}\right), \operatorname{LDB}\left(\mathbf{D}_{1}\right)\right)$ and $\mathbf{D}_{2}=\left(\operatorname{SynFnd}\left(\mathbf{D}_{2}\right), \operatorname{LDB}\left(\mathbf{D}_{2}\right)\right)$ be PF-schemata, and let $f: \mathbf{D}_{1} \rightarrow \mathbf{D}_{2}$ be a PF-morphism.

(a) The elementary congruence on $\mathbf{D}_{1}$ induced by $f$ is the relation $\operatorname{Elt} \operatorname{Congr}(f)$ on $\operatorname{ExtSynFnd}\left(\mathbf{D}_{1}\right)$ given by $(x, y) \in$ EltCongr $(f)$ iff $f^{\sharp}(x)=f^{\sharp}(y)$.

(b) The full syntactic congruence on $\mathbf{D}_{1}$ induced by $f$ is the relation $\operatorname{Syn} \operatorname{Congr}(f)$ on $\mathrm{DB}(\mathbf{D})$ given by $\left(M_{1}, M_{2}\right) \in \operatorname{SynCongr}(f)$ iff $\bar{f}^{\natural}\left(M_{1}\right)=\bar{f}^{\natural}\left(M_{2}\right)$.

(c) The full semantic congruence on $\mathbf{D}_{1}$ induced by $f$ is the relation $\operatorname{Sem} \operatorname{Congr}(f)$ on $\operatorname{LDB}(\mathbf{D})$ given by $\left(M_{1}, M_{2}\right) \in \operatorname{Sem} \operatorname{Congr}(f)$ iff $f^{b}\left(M_{1}\right)=f^{b}\left(M_{2}\right)$.

(d) The full congruence on $\mathbf{D}_{1}$ induced by $f$ is the relation Congr $(f)$ on $\operatorname{ExtSynFnd}\left(\mathbf{D}_{1}\right) \boxplus \operatorname{LDB}\left(\mathbf{D}_{1}\right)$ given by $\left(P_{1}, P_{2}\right) \in \operatorname{Congr}(f)$ iff $f^{\boxplus}\left(P_{1}\right)=f^{\boxplus}\left(P_{2}\right)$.

These four congruences have natural counterparts on views. Let $\Gamma=(\mathbf{V}, \gamma)$ be an PF-view of $\mathbf{D}$.

(e) The elementary congruence of $\Gamma$, denoted $\operatorname{Elt} \operatorname{Congr}(\Gamma)$, is just $\operatorname{Elt} \operatorname{Congr}(\gamma)$.

(f) The syntactic congruence of $\Gamma$, denoted $\operatorname{Syn} \operatorname{Congr}(\Gamma)$, is just $\operatorname{Syn} \operatorname{Congr}(\gamma)$.

(g) The semantic congruence of $\Gamma$, denoted $\operatorname{Sem} \operatorname{Congr}(\Gamma)$, is just $\operatorname{Sem} \operatorname{Congr}(\gamma)$. 
(h) The full congruence of $\Gamma$, denoted $\operatorname{Congr}(\Gamma)$, is just $\operatorname{Congr}(\gamma)$.

The congruences SynCongr $(f)$ and SemCongr $(f)$ have a special structure. Since they are defi ned in terms of EltCongr $(f)$, two sets are equivalent if and only if each element in one is equivalent to an element in the other. In the formalization below, note in particular the use of NullExt(-) (see 3.2) to support the fact that the syntactic foundation $f^{\sharp}$ of a PF-morphism $f$ involves the null element as well as the elements of the syntactic foundation.

3.9 Proposition - Characterization of equivalences Let $\mathbf{D}_{1}=\left(\operatorname{Syn} F \mathrm{nd}\left(\mathbf{D}_{1}\right), \operatorname{LDB}\left(\mathbf{D}_{1}\right)\right)$ and $\mathbf{D}_{2}=\left(\operatorname{Syn} F \operatorname{nd}\left(\mathbf{D}_{2}\right), \operatorname{LDB}\left(\mathbf{D}_{2}\right)\right)$ be PF-schemata, and let $f: \mathbf{D}_{1} \rightarrow \mathbf{D}_{2}$ be a PF-morphism.

(a) For $M_{1}, M_{2} \in \mathrm{DB}(\mathbf{D}),\left(M_{1}, M_{2}\right) \in \operatorname{Syn} \operatorname{Congr}(f)$ iff the following two conditions are satisfied:

(eeq-i) $\left(\forall x \in M_{1}\right)\left(\exists y \in \operatorname{NullExt}\left(M_{2}\right)\right)((x, y) \in \operatorname{EltCongr}(f))$.

(eeq-ii) $\left(\forall y \in M_{2}\right)\left(\exists x \in \operatorname{NullExt}\left(M_{1}\right)\right)((x, y) \in \operatorname{EltCongr}(f))$.

(b) Similarly, for $M_{1}, M_{2} \in \operatorname{LDB}(\mathbf{D}),\left(M_{1}, M_{2}\right) \in \operatorname{Sem} \operatorname{Congr}(f)$ iff the conditions (eeq-i) and (eeq-2) are satisfied.

Proof: Part (a) follows from the fact that $\bar{f}$ is defi ned to be the full extension of $f^{*}$ (see 3.4). Part (b) then follows immediately, since $\operatorname{Sem} \operatorname{Congr}(f) \subseteq \operatorname{Syn} \operatorname{Congr}(f)$.

3.10 Null-augmented congruence containment Given PF-views $\Gamma_{1}=\left(\mathbf{V}_{1}, \gamma_{1}\right)$ and $\Gamma_{2}=\left(\mathbf{V}_{2}, \gamma_{2}\right)$ of the PF-schema $\mathbf{D}$, to say that the congruence of $\Gamma_{1}$ is fi ner than that of $\Gamma_{2} i$ it does not suffi ce to assert only that $\operatorname{Congr}\left(\Gamma_{1}\right) \subseteq \operatorname{Congr}\left(\Gamma_{2}\right)$. It is also necessary to guarantee that whenever $\gamma_{1}^{\sharp}$ maps a particular element to the null element, so too does $\gamma_{2}^{\sharp}$. More precisely, proceed as follows.

(a) Defi ne EltCongr $\left(\Gamma_{1}\right) \stackrel{\oplus}{ \pm} \operatorname{Elt} \operatorname{Congr}\left(\Gamma_{2}\right)$ to mean that $\left(\forall x \in \operatorname{SynFnd}\left(\mathbf{D}_{1}\right)\right)\left(\left(\boldsymbol{\gamma}_{1}^{\sharp}(x)=\eta_{\mathbf{v}_{1}}\right) \Rightarrow\left(\gamma_{2}^{\sharp}(x)=\right.\right.$ $\left.\left.\eta_{\mathbf{v}_{2}}\right)\right)$.

When this condition is appended to a usual set-theoretic containment of congruences, new defi nitions, denoted using $\sqsubseteq$ instead of $\subseteq$, are obtained.

(b) Define EltCongr $\left(\Gamma_{1}\right) \sqsubseteq \operatorname{EltCongr}\left(\Gamma_{2}\right)$ to mean that both $\operatorname{EltCongr}\left(\Gamma_{1}\right) \subseteq \operatorname{EltCongr}\left(\Gamma_{2}\right)$ and EltCongr $\left(\Gamma_{1}\right) \stackrel{\oplus}{ \pm} \operatorname{Elt} \operatorname{Congr}\left(\Gamma_{2}\right)$ hold.

(c) Defi ne SynCongr $\left(\Gamma_{1}\right) \sqsubseteq \operatorname{Syn} \operatorname{Congr}\left(\Gamma_{2}\right)$ to mean that both $\operatorname{Syn} \operatorname{Congr}\left(\Gamma_{1}\right) \subseteq \operatorname{Syn} \operatorname{Congr}\left(\Gamma_{2}\right)$ and EltCongr $\left(\Gamma_{1}\right) \stackrel{\oplus}{E} \operatorname{EltCongr}\left(\Gamma_{2}\right)$ hold.

(d) Defi ne Congr $\left(\Gamma_{1}\right) \sqsubseteq \operatorname{Congr}\left(\Gamma_{2}\right)$ to mean that both $\operatorname{Congr}\left(\Gamma_{1}\right) \subseteq \operatorname{Congr}\left(\Gamma_{2}\right)$ and $\operatorname{Elt} \operatorname{Congr}\left(\Gamma_{1}\right) \subseteq$ EltCongr $\left(\Gamma_{2}\right)$ hold.

Next, the conditions which ensure that the fi ll-in of Fig. 1 exists in the framework of PF-morphisms are established.

3.11 Proposition Let $\mathbf{D}=(\operatorname{SynFnd}(\mathbf{D}), \operatorname{LDB}(\mathbf{D}))$ be a $P F-$ schema, and let $\Gamma_{1}=\left(\mathbf{V}_{1}, \gamma_{1}\right)$ and $\Gamma_{2}=$ $\left(\mathbf{V}_{2}, \gamma_{2}\right)$ be PF-views of $\mathbf{D}$. The following conditions are equivalent.

(a) EltCongr $\left(\Gamma_{1}\right) \sqsubseteq \operatorname{EltCongr}\left(\Gamma_{2}\right)$. 
(b) SynCongr $\left(\Gamma_{1}\right) \sqsubseteq \operatorname{SynCongr}\left(\Gamma_{2}\right)$.

(c) $\operatorname{Congr}\left(\Gamma_{1}\right) \sqsubseteq \operatorname{Congr}\left(\Gamma_{2}\right)$.

(d) There is an open surjective PF-morphism $f: \mathbf{V}_{1} \rightarrow \mathbf{V}_{2}$ with the property that $\gamma_{2}=f \circ \gamma_{1}$.

PROOF: The equivalence of conditions (a)-(c), as well as (d) $\Rightarrow$ (c), are immediate.

Suppose that conditions (a)-(c) are satisfi ed. Defi ne $g: \operatorname{SynFnd}(\mathbf{Y}) \rightarrow \operatorname{SynFnd}\left(\mathbf{V}_{2}\right)$ as follows. For $y \in \operatorname{SynFnd}\left(\mathbf{V}_{1}\right)$, pick any $x \in \operatorname{SynFnd}(\mathbf{D})$ with $\gamma_{1}^{\sharp}(x)=y$, and defi ne $g(y)=\psi_{2}(x)$. The fact that EltCongr $\left(\Gamma_{1}\right) \subseteq$ EltCongr $\left(\Gamma_{2}\right)$ ensures that this defi nition is independent of the particular choice of $x \in\left(\gamma_{1}^{\sharp}\right)^{-1}(y)$. Now, simply defi ne $f$ to be the unique PF-morphism with $f^{\sharp}=g$. The fact that $\left(\forall x \in \operatorname{SynFnd}\left(\mathbf{D}_{1}\right)\right)\left(\left(\gamma_{1}^{\sharp}(x)=\eta_{\mathbf{D}_{1}}\right) \Rightarrow\left(\gamma_{2}^{\sharp}(x)=\eta_{\mathbf{D}_{2}}\right)\right)$ ensures that $f$ will be surjective. To see that $f^{b}: \operatorname{LDB}\left(\mathbf{V}_{1}\right) \rightarrow \operatorname{LDB}\left(\mathbf{V}_{2}\right)$ is open, let $N_{1}, N_{2} \in \operatorname{LDB}\left(\mathbf{V}_{2}\right)$ with $N_{1} \subseteq N_{2}$, and choose $M_{1}, M_{2} \in \operatorname{LDB}(\mathbf{D})$ with $M_{1} \subseteq N_{2}$ and $\gamma_{2}^{\prime}\left(M_{1}\right)=N_{1}, \gamma_{2}^{\prime}\left(M_{2}\right)=N_{2}$. Then $\gamma_{2}^{\prime}\left(M_{1}\right)=f^{\prime}\left(\gamma_{1}^{\prime}\left(M_{1}\right)\right) \subseteq f^{\prime}\left(\gamma_{1}^{\prime}\left(M_{2}\right)\right)=\gamma_{2}^{\prime}\left(M_{2}\right)$, so $\left(\gamma_{1}^{b}\left(M_{1}\right), \gamma_{2}^{b}\left(M_{2}\right)\right)$ is the desired pair which maps to $\left(N_{1}, N_{2}\right)$ under $f^{b}$.

3.12 Relative views Let $\mathbf{D}=(\operatorname{SynFnd}(\mathbf{D}), \operatorname{LDB}(\mathbf{D}))$ be a PF-schema, and let $\Gamma_{1}=\left(\mathbf{V}_{1}, \gamma_{1}\right)$ and $\Gamma_{2}=\left(\mathbf{V}_{2}, \gamma_{2}\right)$ be PF-views of $\mathbf{D}$. Suppose further that the equivalent conditions of 3.11 are satisfi ed. The relative view from $\Gamma_{1}$ to $\Gamma_{2}$ is the PF-view $\Lambda\left(\Gamma_{1}, \Gamma_{2}\right)=\left(\mathbf{V}_{2}, \lambda\left\langle\Gamma_{1}, \Gamma_{2}\right\rangle\right)$ of $\mathbf{V}_{1}$, with $\lambda\left\langle\Gamma_{1}, \Gamma_{2}\right\rangle: \mathbf{V}_{1} \rightarrow \mathbf{V}_{2}$ the unique fi llin identifi ed in 3.11(d) above. This situation is depicted in the diagram to the right. The concept of a relative view is particularly critical to the construction of a meet, as illustrated in Fig. 2, and developed in 4.3.

As a concrete example, think of $\mathbf{D}$ as $R[A B C], \mathbf{V}_{1}$ as $R[A B]$, and $\mathbf{V}_{2}$ as

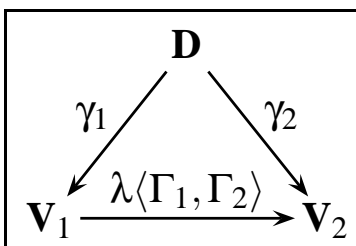

Figure 3: Relative view morphism $R[B]$, with $\gamma_{1}=\pi_{A B}: R[A B C] \rightarrow R[A B]$, and $\gamma_{2}=\pi_{B}: R[A B C] \rightarrow R[B]$. Then $\lambda\left\langle\Gamma_{1}, \Gamma_{2}\right\rangle=\pi_{B}: R[A B] \rightarrow$ $R[B]$.

3.13 The view defined by an elementary congruence In some important constructions, it is necessary to construct a view from a congruence. (In this work, the meet is such a construction; see 4.3(c)) for details.) This is a rather straightforward process; the steps are outlined below.

Let (SynFnd $(\mathbf{D}), \operatorname{LDB}(\mathbf{D}))$ be a PF-schema, and let $R$ be an equivalence relation on $\operatorname{ExtSynFnd}(\mathbf{D})$. A PF-view of $\mathbf{D}$, denoted $\llbracket \mathbf{D} \rrbracket / / R$ and unique up to isomorphism, with the property that the elementary equivalence of the view is $R$, may be constructed via the following steps.

(a) Defi ne $R_{\text {h }}=R_{\mid \operatorname{SynFnd}(\mathbf{D})}=R \cap(\operatorname{SynFnd}(\mathbf{D}) \times \operatorname{SynFnd}(\mathbf{D}))$.

(b) Defi ne SynFnd $(\llbracket \mathbf{D} \rrbracket / / R)=\operatorname{SynFnd}(\mathbf{D}) / R_{\text {h }}=$ the set of equivalence classes of SynFnd $(\mathbf{D})$ under the equivalence relation $R_{\mathfrak{n}}$.

(c) For $M \in \mathrm{DB}(\mathbf{D})$, defi ne $[M]_{R_{\eta}}=\left\{[x]_{R_{\eta}} \mid x \in M\right.$ and $\left.\left(x, \eta_{\mathbf{D}}\right) \notin R\right\}$. ([x] $]_{R_{\eta}}$ denotes the block of SynFnd $(\mathbf{D}) / R_{\eta}$ in which $x$ lies.)

(d) Defi ne $\operatorname{LDB}(\llbracket \mathbf{D} \rrbracket / / R)=\left\{\left[M_{R_{\text {凡 }}} \mid M \in \operatorname{LDB}(\mathbf{D})\right\}\right.$.

(e) Defi ne $\llbracket \mathbf{D} \rrbracket / / R=(\operatorname{SynFnd}(\llbracket \mathbf{D} \rrbracket / / R), \llbracket \operatorname{LDB}(\mathbf{D}) \rrbracket / / R)$.

It is easy to see that $\llbracket \mathbf{D} \rrbracket / / R$ is a PF-schema. 
(f) Defi ne $g$ : ExtSynFnd(D) $\rightarrow$ ExtSynFnd $(\llbracket \mathbf{D} \rrbracket / / R)$ on elements as follows.

$$
x \mapsto \begin{cases}{[x]_{R_{\bigvee}}} & \text { if }\left(x, \eta_{\mathbf{D}}\right) \notin R . \\ \eta_{\llbracket \mathbf{D} \rrbracket / \mathbf{R}} & \text { if }\left(x, \eta_{\mathbf{D}}\right) \in R .\end{cases}
$$

(g) Let $\llbracket \theta \rrbracket_{R}: \mathbf{D} \rightarrow \llbracket \mathbf{D} \rrbracket / / R$ be the PF-morphism with $\left(\llbracket \theta \rrbracket_{R}\right)^{\sharp}=g$.

(h) Finally, put $\llbracket \Theta \rrbracket_{R}=\left(\llbracket \mathbf{D} \rrbracket / / R, \llbracket \theta \rrbracket_{R}\right)$.

It is easy to see that $\llbracket \Theta \rrbracket_{R}$ is a PF-view which has $R$ as its elementary congruence.

\section{Decompositions in the Context of PF-Views}

In order to be able to use PF-views as the abstract model for meet-complementary pairs, it is necessary to show that the key ideas which were developed in the earlier work [Heg04a] can be extended to this framework. Although the main ideas are essentially the same, there are a number of details to be considered, particularly as regards the congruence to use in defi ning complementary pairs.

4.1 Products and complements of views Let $\Gamma_{1}=\left(\mathbf{V}_{1}, \gamma_{1}\right)$ and $\Gamma_{2}=\left(\mathbf{V}_{2}, \gamma_{2}\right)$ be PF-views of the PF-schema D. Unfortunately, there does not appear to be a clean way to extend the defi nition of view product presented in [Heg04a, 2.1] to the PF-context in such a way that $\gamma_{1} \otimes \gamma_{2}$ is a PF-morphism. The problem is that the product mapping cannot be defi ned by an elementary mapping, since a single element in the main schema $\mathbf{D}$ maps to two elements in the decomposition, one in $\operatorname{LDB}\left(\mathbf{V}_{1}\right)$ and the other in $\operatorname{LDB}\left(\mathbf{V}_{2}\right)$. Fortunately, this is not a serious problem. There is no need to require the decomposition mapping to be a PF-morphism; it is quite suffi cient that it be a poset morphism, and so the defi nitions of the earlier work suffi ce. The appropriate translations of these defi nitions are as follows.

(a) Defi ne $\operatorname{LDB}\left(\mathbf{V}_{1 \gamma_{1}} \otimes_{\gamma_{2}} \mathbf{V}_{2}\right)=\left\{\left(\gamma_{1}^{\prime}(M), \gamma_{2}^{\prime}(M)\right) \mid M \in \operatorname{LDB}(\mathbf{D})\right\}$.

(b) Defi ne the function $\gamma_{1} \otimes \gamma_{2}^{b}: \operatorname{LDB}(\mathbf{D}) \rightarrow \operatorname{LDB}\left(\mathbf{V}_{1 \gamma_{1}} \otimes_{\gamma_{2}} \mathbf{V}_{2}\right)$ by $M \mapsto\left(\gamma_{1}^{b}(M), \gamma_{2}^{b}(M)\right)$.

(c) The pair $\left\{\Gamma_{1}, \Gamma_{2}\right\}$ of PF-views is said to form a subdirect complementary pair just in case $\gamma_{1}^{b} \otimes \gamma_{2}^{b}$ : $\operatorname{LDB}(\mathbf{D}) \rightarrow \operatorname{LDB}\left(\mathbf{V}_{1 \gamma_{1}} \otimes_{\gamma_{2}} \mathbf{V}_{2}\right)$ is a poset isomorphism, with both sets carrying the natural order defi ned by set inclusion $\subseteq$. In this case, it is also said that $\Gamma_{1}$ and $\Gamma_{2}$ are subdirect complements of one another.

In the more general case of order schemata, it is quite possible for $\gamma_{1}^{b} \otimes \gamma_{2}^{b}$ to be a bijection without being a poset isomorphism [Heg04a, 2.11]. However, in the context of PF-views, this is not possible, as is established by the following proposition.

4.2 Proposition Let $\mathbf{D}=(\operatorname{Syn} F \mathrm{nd}(\mathbf{D}), \operatorname{LDB}(\mathbf{D}))$ be a PF-schema, and let $\left\{\Gamma_{1}, \Gamma_{2}\right\}$ be a pair of PF-views of D. For $\left\{\Gamma_{1}, \Gamma_{2}\right\}$ to be a subdirect complementary pair it is necessary and sufficient that $\gamma_{1}^{b} \otimes \gamma_{2}^{b}$ be injective.

PROOF: Since $\gamma_{1}^{b} \otimes \gamma_{2}^{b}$ is surjective by construction, under the injectivity assumption it is bijective. It is immediate that it is also a poset morphism. That $\left(\gamma_{1}^{b} \otimes \gamma_{2}^{b}\right)^{-1}$ is a poset morphism is very straightforward also. Indeed, for $M, N \in \operatorname{LDB}(\mathbf{D})$ with $\gamma_{1}^{b}(M) \subseteq \gamma_{1}^{p}(N)$ and $\gamma_{2}^{\prime}(M) \subseteq \gamma_{2}^{b}(N)$, it is immediate from the defi nition of product ordering that $M \subseteq N$. 
4.3 Fully commuting views and meet complements The importance of commuting congruences cannot be overstated. It is the condition on a complementary pair which ensures that constantcomplement update strategies are independent of the state of the complement [Heg04a, 3.10]. In addition, commuting congruences play a key rôle in the characterization of "desirable" properties of decompositions which are related to the classical notion of acyclic schemata [Heg93].

Because of the way in which the (full) congruence of a PF-view is defi ned, the formal defi nition is identical to that for order views.

(a) The pair $\left\{\Gamma_{1}, \Gamma_{2}\right\}$ of PF-views of the PF-schema $\mathbf{D}$ is called a fully commuting pair if $\operatorname{Congr}\left(\Gamma_{1}\right) \circ$ Congr $\left(\Gamma_{2}\right)=\operatorname{Congr}\left(\Gamma_{2}\right) \circ \operatorname{Congr}\left(\Gamma_{1}\right)$, with "०" denoting ordinary composition of binary relations, as already sketched in 2.1 for set-based views. In view of the defi nitions of 3.8 , this is equivalent to simultaneous satisfaction of the following two conditions:

(i) $\operatorname{Elt} \operatorname{Congr}\left(\Gamma_{1}\right) \circ \operatorname{Elt} \operatorname{Congr}\left(\Gamma_{2}\right)=\operatorname{Elt} \operatorname{Congr}\left(\Gamma_{2}\right) \circ \operatorname{Elt} \operatorname{Congr}\left(\Gamma_{1}\right)$.

(ii) SemCongr $\left(\Gamma_{1}\right) \circ \operatorname{Sem} \operatorname{Congr}\left(\Gamma_{2}\right)=\operatorname{Sem} \operatorname{Congr}\left(\Gamma_{2}\right) \circ \operatorname{Sem} \operatorname{Congr}\left(\Gamma_{1}\right)$.

Of course, if the semantic congruences SemCongr $\left(\Gamma_{1}\right)$ and $\operatorname{Sem} \operatorname{Congr}\left(\Gamma_{2}\right)$ are suffi ciently rich; that is, if they contain enough information to reconstruct the corresponding elementary congruences, then condition (ii) suffi ces. This will be the case if the schemata and views are defi nable as CFA-schemata and CFA-views, but it is not true in general. This provides the motivation for the rather heavy constructions of combining the two components using the " $\boxplus$ " operators.

(b) A subdirect complementary pair $\left\{\Gamma_{1}, \Gamma_{2}\right\}$ which is fully commuting is called a meetcomplementary pair, and $\Gamma_{1}$ and $\Gamma_{2}$ are called meet complements of one another.

(c) If $\left\{\Gamma_{1}, \Gamma_{2}\right\}$ is a meet-complementary pair of PF-views of the PF-schema $\mathbf{D}$, the view (unique up to isomorphism - see 3.13) whose congruence is $\operatorname{Congr}\left(\Gamma_{1}\right) \circ \operatorname{Congr}\left(\Gamma_{2}\right)$ is called the meet of $\left\{\Gamma_{1}, \Gamma_{2}\right\}$, and is denoted $\Gamma_{1} \wedge \Gamma_{2}$. It is immediate that $\operatorname{Congr}\left(\Gamma_{i}\right) \sqsubseteq \operatorname{Congr}\left(\Gamma_{1} \wedge \Gamma_{2}\right)$ for $i \in\{1,2\}$.

4.4 Independence dependencies The meet of a pair of meet-complementary views defi nes the common ground on which the views must agree. More formally, let $\mathbf{D}=(\operatorname{SynFnd}(\mathbf{D}), \mathrm{LDB}(\mathbf{D}))$ be a PF-schema, let $\left\{\Gamma_{1}, \Gamma_{2}\right\}$ be a subdirect complementary pair of PF-views, and let $\Gamma_{3}=\left(\mathbf{V}_{3}, \gamma_{3}\right)$ be a PF-view of $\mathbf{D}$, with $\operatorname{Congr}\left(\Gamma_{1}\right) \sqsubseteq \operatorname{Congr}\left(\Gamma_{3}\right)$ and $\operatorname{Congr}\left(\Gamma_{2}\right) \sqsubseteq \operatorname{Congr}\left(\Gamma_{3}\right)$.

(a) The $\Gamma_{3}$-independence dependency on $\mathbf{V}_{1 \gamma_{1}} \otimes_{\gamma_{2}} \mathbf{V}_{2}$, denoted $\otimes_{\Gamma_{3}}$, is satisfi ed iff for any $M_{1} \in$ $\operatorname{LDB}\left(\mathbf{V}_{1}\right)$ and $M_{2} \in \operatorname{LDB}\left(\mathbf{V}_{2}\right)$, the following condition is satisfi ed [Heg04a, 2.13].

$$
\left(\left(M_{1}, M_{2}\right) \in \operatorname{LDB}\left(\mathbf{V}_{1 \gamma_{1}} \otimes_{\gamma_{2}} \mathbf{V}_{2}\right)\right) \Leftrightarrow\left(\lambda\left\langle\Gamma_{1}, \Gamma_{3}\right\rangle\left(M_{1}\right)=\lambda\left\langle\Gamma_{2}, \Gamma_{3}\right\rangle\left(M_{2}\right)\right)
$$

In the context of PF-schemata, this dependency may be expressed in an element-by-element fashion. To understand the difference, fi rst consider the example of a simple relational schema $R[A B C]$ constrained by the single FD $B \rightarrow C$, which decomposes losslessly and in a dependency-preserving fashion into the two projections $\Pi_{A B}$ and $\Pi_{B C}$. In this case, the meet $\Pi_{A B} \wedge \Pi_{B C}=\Pi_{B}$ [Heg04a, 2.17]. The $\Pi_{B}$-independence dependency asserts that the projection of the state of each view on attribute $B$ is the same. The alternate, element-by-element characterization states that for each tuple $(a, b)$ in the state of the view $\Pi_{A B}$, there is a tuple $(b, c)$ in the state of the view $\Pi_{B C}$ with a matching $B$-value, and conversely. These conditions are so obviously identical that it may seem pointless to differentiate between them. However, in a more general context, they display an important difference. The 
$\Pi_{B}$-dependency characterization is formulated within the very general framework of order views; no concept of tuple is necessary. On the other hand, a generalization of the tuple-by-tuple matching condition requires a corresponding abstraction of the notion of a tuple; while general order views do not support this abstraction, PF-views do. The formalizations are as follows.

(b) The pointwise $\Gamma_{3}$-independence dependency is satisfi ed iff the following two dual conditions are met for each $\left(M_{1}, M_{2}\right) \in \operatorname{LDB}\left(\mathbf{V}_{1 \gamma_{1}} \otimes_{\gamma_{2}} \mathbf{V}_{2}\right)$.

$$
\begin{aligned}
& \left(\forall x_{1} \in M_{1}\right)\left(\exists x_{2} \in \operatorname{NullExt}\left(M_{2}\right)\right)\left(\lambda\left\langle\Gamma_{1}, \Gamma_{3}\right\rangle^{\sharp}\left(x_{1}\right)=\lambda\left\langle\Gamma_{2}, \Gamma_{3}\right\rangle^{\sharp}\left(x_{2}\right)\right) \\
& \left(\forall x_{2} \in M_{2}\right)\left(\exists x_{1} \in \operatorname{NullExt}\left(M_{1}\right)\right)\left(\lambda\left\langle\Gamma_{1}, \Gamma_{3}\right\rangle^{\sharp}\left(x_{1}\right)=\lambda\left\langle\Gamma_{2}, \Gamma_{3}\right\rangle^{\sharp}\left(x_{2}\right)\right)
\end{aligned}
$$

In other words, in the context of PF-views, conditions (id:1) and (id:2) may replace (id).

The formalization of these ideas is recorded in the following proposition.

4.5 Proposition Let (SynFnd $(\mathbf{D}), \operatorname{LDB}(\mathbf{D}))$ be a PF-schema, let $\left\{\Gamma_{1}, \Gamma_{2}\right\}$ be a subdirect complementary pair of $P F$-views of $\mathbf{D}$, and let $\Gamma_{3}$ be the view (unique up to isomorphism) whose congruence is the smallest equivalence relation on $\operatorname{ExtSynFnd}\left(\mathbf{D}_{1}\right) \boxplus \operatorname{LDB}\left(\mathbf{D}_{1}\right)$ containing both $\operatorname{Congr}\left(\Gamma_{1}\right)$ and Congr $\left(\Gamma_{2}\right)$. Then $\left\{\Gamma_{1}, \Gamma_{2}\right\}$ is a meet-complementary pair iff conditions (id:1) and (id:2) of 4.4 are satisfied.

PROOF: Follows directly from the discussion of 4.4 and [Heg04a, 2.14].

4.6 Generalized join dependencies The constructions of 4.4 characterize completely the conditions needed for a decomposition into two views to be independent relative to a common view, but they do not specify completely the minimal constraints on the main schema $\mathbf{D}$ which enable that decomposition. Consider, once again, the relational schema $R[A B C]$, this time without the FD $B \rightarrow C$, decomposed into the two projection views $\Pi_{A B}$ and $\Pi_{B C}$, let $r_{1}=\left\{\left(a_{1}, b_{1}, c_{1}\right),\left(a_{2}, b_{1}, c_{2}\right)\right\}$, and let $r_{2}=\left\{\left(a_{1}, b_{1}, c_{1}\right),\left(a_{1}, b_{1}, c_{2}\right),\left(a_{2}, b_{1}, c_{1}\right),\left(a_{2}, b_{1}, c_{2}\right)\right\}$ be relations on $R[A B C]$. Both $r_{1}$ and $r_{2}$ map to $\left(\left\{\left(a_{1}, b_{1}\right),\left(a_{2}, b_{1}\right)\right\},\left\{\left(b_{1}, c_{1}\right),\left(b_{1}, c_{2}\right)\right\}\right)$ under the decomposition into the $A B$ - and $B C$-projections, but only one may be a legal state of $R[A B C]$ under the constraints of the schema if the decomposition is to be lossless. The classical relational theory mandates that the join dependency $\bowtie[A B, B C]$ hold on $R[A B C]$, so that $r_{1}$ is excluded. However, the general theory outlined in 4.4 makes no such preference. There could just as easily be a complex set of constraints on $R[A B C]$ under which $r_{1}$ is legal but $r_{2}$ is not.

In the general decomposition context developed in [Heg04a], it does not appear to be possible to express such a preference in a natural way. However, in the present context, in which database states are expressed as sets of elements, it is quite possible. Specifi cally, return to the general context of a PF-schema $\mathbf{D}=(\operatorname{SynFnd}(\mathbf{D}), \operatorname{LDB}(\mathbf{D}))$, with a pair $\left\{\Gamma_{1}, \Gamma_{2}\right\}$ of meet-complementary PF-views whose meet is $\Gamma_{3}$.

(a) The join completion of $M \in \mathrm{DB}(\mathbf{D})$ relative to $\left\{\Gamma_{1}, \Gamma_{2}\right\}$ is

$$
\operatorname{JoinCompl}_{\left\langle\Gamma_{1} ; \Gamma_{2}\right\rangle}(M)=\left\{x \in \operatorname{SynFnd}(\mathbf{D}) \mid(\exists y \in M)(\exists z \in M)\left(\gamma_{1}^{\sharp}(x)=\gamma_{1}^{\sharp}(y) \wedge \gamma_{2}^{\sharp}(x)=\gamma_{2}^{\sharp}(z)\right)\right\}
$$

(b) Call $M \in \mathrm{DB}(\mathbf{D})$ join complete relative to $\left\{\Gamma_{1}, \Gamma_{2}\right\}$ if $M=\operatorname{JoinCompl}_{\left\langle\Gamma_{1} ; \Gamma_{2}\right\rangle}(M)$. It is easy to see that $M$ is join complete iff the following generalized join dependency, denoted $\bowtie\left[\Gamma_{1}, \Gamma_{2}\right]$, is satisfi ed. 
(gjd) $(\forall x, y \in M)\left(\left(\gamma_{3}^{\sharp}(x)=\gamma_{3}^{\sharp}(y)\right) \Rightarrow(\exists z \in M)\left(\gamma_{1}^{\sharp}(z)=\gamma_{1}^{\sharp}(x) \wedge\left(\gamma_{2}^{\sharp}(z)=\gamma_{2}^{\sharp}(y)\right)\right)\right)$

(c) Say that $\mathbf{D}$ uses join reconstruction from $\left\{\Gamma_{1}, \Gamma_{2}\right\}$ if every $M \in \operatorname{LDB}(\mathbf{D})$ is join complete relative to $\left\{\Gamma_{1}, \Gamma_{2}\right\}$.

The theory of this paper will generally be formulated under the explicit stipulation that the decomposition of the main schema $\mathbf{D}$ is governed by the join reconstruction from $\left\{\Gamma_{1}, \Gamma_{2}\right\}$. In such a context, it is necessary to work with databases which, while not necessary in $\operatorname{LDB}(\mathbf{D})$, have the property that their join completions are legal. The formal defi nition is as follows.

(d) Call $M \in \mathrm{DB}(\mathbf{D})$ a join premodel relative to $\left\langle\Gamma_{1} ; \Gamma_{2}\right\rangle$ if JoinCompl $\Gamma_{\left.\Gamma_{1} ; \Gamma_{2}\right\rangle}(M) \in \operatorname{LDB}(\mathbf{D})$. The set of all join premodels of $\mathbf{D}$ relative to JPair $\left\langle\Gamma_{1} ; \Gamma 2\right\rangle$ is denoted $\operatorname{PLDB}\left(\left\langle\overline{\mathbf{D}} ;\left\{\Gamma_{1}, \Gamma_{2}\right\}\right\rangle\right)$.

(e) The schema $\mathbf{D}$ has implicit join completion with respect to $\left\{\Gamma_{1}, \Gamma_{2}\right\}$ if whenever $M \in \mathrm{DB}(\mathbf{D})$ has the property that $\bar{\gamma}_{i}(M) \in \operatorname{LDB}\left(\mathbf{V}_{i}\right)$ for both $i=1$ and $i=2$, then $M \in \operatorname{LDB}(\mathbf{D})$; i.e., $M=$ JoinCompl $_{\left\langle\Gamma_{1} ; \Gamma_{2}\right\rangle}(M)$.

For example, the schema $\mathbf{E}_{2}$ of 1.2 has implicit join completion, because the join dependency $\bowtie[A B C E, A B C D]$ is implied by the FD $A B C \rightarrow E$, and the latter is satisfi ed by any legal state of the schema of $\Pi_{A B C E}$.

4.7 Observation - Premodels and view axiomatization Let $\mathbf{D}$ be a PF-schema with $\left\{\Gamma_{1}, \Gamma_{2}\right\}$ a meet-complementary pair of $P F$-views of $\mathbf{D}$. Assume further that $\mathbf{D}$ has implicit join completion with respect to $\left\{\Gamma_{1}, \Gamma_{2}\right\}$. Then for any $M \in \mathrm{DB}(\mathbf{D}), M \in \operatorname{PLDB}\left(\left\langle\overline{\mathbf{D}} ;\left\{\Gamma_{1}, \Gamma_{2}\right\}\right\rangle\right)$ iff $\bar{\gamma}_{i}(M) \in \operatorname{LDB}\left(\mathbf{V}_{i}\right)$ for both $i=1$ and $i=2$.

4.8 Join schemata In order to study the complexity of constraint satisfaction and updates, it is necessary to extend the semantic views; i.e., those views which operate on collections of the form $\operatorname{LDB}(-)$, to syntactic views; i.e., those which operate on collections of the from $\mathrm{DB}(-)$. As identifi ed in 3.4(h) and 3.7(b), the information needed to effect this translation is built into the PF-view itself. However, there is one important detail which cannot be overlooked; namely, if $\left\{\Gamma_{1}, \Gamma_{2}\right\}$ is a meetcomplementary pair of PF-views on the PF-schema $\mathbf{D}$, it is not generally the case that $\left\{\bar{\Gamma}_{1}, \bar{\Gamma}_{2}\right\}$ is a meet-complementary pair on $\overline{\mathbf{D}}$. The problem is that $\bar{\gamma}_{1} \otimes \bar{\gamma}_{2}^{\prime}: \overline{\mathbf{D}} \rightarrow \overline{\mathbf{V}}_{1 \bar{\gamma}_{11}} \otimes_{\bar{\gamma}_{2}} \overline{\mathbf{V}}_{2}$ is not necessarily injective, although it is certainly surjective. To render it injective, the domain must be restricted to those elements of $\operatorname{DB}(\mathbf{D})$ which are join complete, in the sense of 4.6(b) above.

More formally, let $\mathbf{D}=(\operatorname{SynFnd}(\mathbf{D}), \operatorname{LDB}(\mathbf{D}))$ be a PF-schema, and let $\left\{\Gamma_{1}, \Gamma_{2}\right\}$ be a meet complementary pair of views of $\mathbf{D}$. Assume further that $\mathbf{D}$ uses join reconstruction from $\left\{\Gamma_{1}, \Gamma_{2}\right\}$.

(a) Defin ne $\left.\overline{\mathbf{D}} ;\left\{\Gamma_{1}, \Gamma_{2}\right\}\right\rangle$ to be the schema with $\operatorname{SynFnd}\left(\left\langle\overline{\mathbf{D}} ;\left\{\Gamma_{1}, \Gamma_{2}\right\}\right\rangle\right)=\operatorname{SynFnd}(\mathbf{D})$ and $\operatorname{LDB}\left(\left\langle\overline{\mathbf{D}} ;\left\{\Gamma_{1}, \Gamma_{2}\right\}\right\rangle\right)=\left\{\operatorname{JoinCompl}_{\left\langle\Gamma_{1} ; \Gamma_{2}\right\rangle}(M) \mid M \in \operatorname{DB}(\mathbf{D})\right\}$. The notation $\operatorname{JDB}_{\left\langle\Gamma_{1} ; \Gamma_{2}\right\rangle}(\overline{\mathbf{D}})$ will often be used to denote $\operatorname{LDB}\left(\left\langle\overline{\mathbf{D}} ;\left\{\Gamma_{1}, \Gamma_{2}\right\}\right\rangle\right)$.

(b) The view $\widehat{\Gamma}_{i}=\left(\overline{\mathbf{V}}_{i}, \widehat{\gamma}_{i}\right)(i \in\{1,2\})$ of $\left\langle\overline{\mathbf{D}} ;\left\{\Gamma_{1}, \Gamma_{2}\right\}\right\rangle$ is given by $\widehat{\gamma}_{i}=\bar{\gamma}_{i\left|J \mathrm{JB}_{\left\langle\Gamma_{1} ; \Gamma_{2}\right\rangle}\right\rangle}$; $)$ that is, $\widehat{\gamma}_{i}$ is just $\bar{\gamma}_{i}$ restricted to the join-complete members of $\mathrm{DB}(\mathbf{D})$.

It is easy to see that $\widehat{\gamma}_{i}$ remains surjective, since for any $M \in \mathrm{DB}(\mathbf{D}), \bar{\gamma}_{i}(M)=\bar{\gamma}_{i}\left(\operatorname{JoinCompl}_{\left\langle\Gamma_{1} ; \Gamma_{2}\right\rangle}(M)\right)$. 
It should also be stressed that the "hat" notation; e.g., $\widehat{\gamma}_{i}$, is ambiguous, since the defi nition depends not only upon $\gamma_{i}$, but upon the meet-complementary pair $\left\{\Gamma_{1}, \Gamma_{2}\right\}$ as well. However, if this pair is clearly fi xed by context, then the meaning of things such as $\widehat{\gamma}_{i}$ will be apparent and unambiguous.

It is also useful to have some terminology which describes pairs, and sets of pairs, which are compatible with respect to a decomposition.

(c) Call a pair $\left(M_{1}, M_{2}\right) \in \mathrm{DB}\left(\mathbf{V}_{1}\right) \times \mathrm{DB}\left(\mathbf{V}_{2}\right)$ join compatible for $\left\langle\Gamma_{1} ; \Gamma_{2}\right\rangle$ if there is an $M \in$ $\mathrm{JDB}_{\left\langle\Gamma_{1} ; \Gamma_{2}\right\rangle}(\overline{\mathbf{D}})$ such that $\left(\hat{\gamma}_{1}^{\prime} \otimes \hat{\gamma}_{2}\right)(M)=\left(M_{1}, M_{2}\right)$.

4.9 Proposition Let $(\operatorname{Syn} F \mathrm{nd}(\mathbf{D}), \operatorname{LDB}(\mathbf{D}))$ be a PF-schema, and let $\left\{\Gamma_{1}, \Gamma_{2}\right\}$ be a meetcomplementary pair of PF-views over $\mathbf{D}$. Assume further that $\mathbf{D}$ uses join reconstruction from $\left\{\Gamma_{1}, \Gamma_{2}\right\}$.

(a) $\left\{\widehat{\Gamma}_{1}, \widehat{\Gamma}_{2}\right\}$ is a meet complementary pair of $P F$-views over $\left\langle\overline{\mathbf{D}} ;\left\{\Gamma_{1}, \Gamma_{2}\right\}\right\rangle$.

(b) $\hat{\gamma}_{1}^{b} \otimes \hat{\gamma}_{2}=\left(\bar{\gamma}_{1} \otimes \bar{\gamma}_{2}^{b}\right)_{\mid \mathrm{JDB}_{\left\langle\Gamma_{1} ; \Gamma_{2}\right\rangle}(\overline{\mathbf{D}})}$, with the last entry denoting the restriction of $\bar{\gamma}_{1} \otimes \bar{\gamma}_{2}$ to the domain $\operatorname{JDB}_{\left\langle\Gamma_{1} ; \Gamma_{2}\right\rangle}(\overline{\mathbf{D}})$.

Proof: To show (a), fi rst note that since $\operatorname{JDB}_{\left\langle\Gamma_{1} ; \Gamma_{2}\right\rangle}(\overline{\mathbf{D}})$ consists entirely of join complete sets,

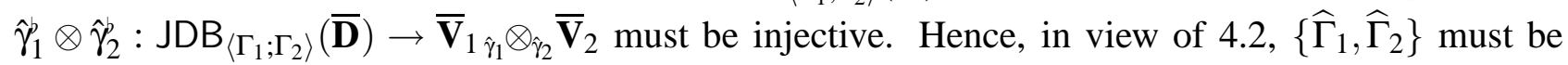
a subdirect complementary pair. To show that it is meet complementary, it suffi ces to observe that for $M \in \operatorname{JDB}_{\left\langle\Gamma_{1} ; \Gamma_{2}\right\rangle}(\overline{\mathbf{D}})$, letting $M_{1}=\widehat{\gamma}_{1}(M)$ and $M_{2}=\widehat{\gamma}_{2}(M)$, conditions (id:1) and (id:2) of 4.4 are satisfi ed, and so by $4.5,\left\{\widehat{\Gamma}_{1}, \widehat{\Gamma}_{2}\right\}$ is a meet-complementary pair.

Part (b) follows directly from 4.8(c)

\section{Complexity of View Constraints in a General Setting}

In this section, the relative complexity of verifying the constraints on a schema via a meetcomplementary pair of its views is investigated. For schemata governed by consistency constraints which do not involve the generation of new elements, the conclusion is strong and positive: in a general sense, a cover of the constraints on the main schema embeds in the view, and so the complexity of constraint checking via the views is no more complex than constraint checking on the main schema itself. This extends the result which was reported in [Heg04b], and this is accomplished with a much simpler proof technique.

For constraints which can generate new tuples (such a join dependencies), the results require a condition called meet uniformity, which stipulates that the two views comprising the decomposition treat this common sub-view in a uniform way. While this condition is somewhat strict, the constructions nonetheless provide valuable insights.

5.1 Notational convention Throughout this section, unless noted specifi cally to the contrary, $\mathbf{D}=$ (SynFnd $(\mathbf{D}), \operatorname{LDB}(\mathbf{D}))$ will be taken to be a PF-schema.

5.2 The $k$-submodel property In the earlier work [Heg04b], the $k$-submodel property was used as the measure of constraint complexity. To motivate the extended framework developed in this section, it is instructive to provide fi rst a brief summary of this idea. 
Given a relation $M$ and a set $\mathcal{F}$ of FDs, it may be determined whether $M$ satisfi es those FDs by checking two tuples at a time. If $M$ does not satisfy all of the dependencies in $\mathcal{F}$, then there must be some $\varphi \in \mathcal{F}$ and some two-element subset $N \subseteq M$ with the property that $\varphi$ does not hold on $N$. In other words, $M$ satisfi es $\mathcal{F}$ iff every subset of $M$ of size two satisfi es $\mathcal{F}$. The $k$-submodel property generalizes this idea of reducing constraint satisfaction to checking satisfaction on subinstances of a fi xed size.

Formally, for $k \in \mathbb{N}$, a $k$-model of $\mathbf{D}$ is any $M \in \mathrm{DB}(\mathbf{D})$ with the property that for every $N \subseteq M$ with $\operatorname{Card}(N) \leq k$, it is necessarily the case that $N \in \operatorname{LDB}(\mathbf{D})$. The schema $\mathbf{D}$ has the $k$-submodel property if for every $M \in \mathrm{DB}(\mathbf{D}), M \in \operatorname{LDB}(\mathbf{D})$ iff $M$ is a $k$-model of $\mathbf{D}$. If $\mathbf{D}$ has the $k$-submodel property, then it has the unrestricted submodel property as well, in the sense that if $M \in \operatorname{LDB}(\mathbf{D})$, then so too is every subset of $M$ [Heg04b, 3.7].

In particular, a relational schema constrained solely by FDs has the 2-submodel property. More generally, the equality-generating constraints, or EGDs, of this classical relational framework, are characterizable via the $k$-submodel property. See 6.1 and 6.2 for more details.

Unfortunately, the $k$-submodel property cannot model any sort of relational constraint which mandates the existence of new tuples based upon existing ones. This includes both full tuplegenerating dependencies (TGDs) and embedded TGDs, such as inclusion dependencies, of which the immensely important foreign-key constraints are a special case.

In order to model these more general constraints, an expanded notion of complexity is introduced. Rather than simply asking whether every subset of $M$ of a given size is a model, it asks whether every subset of $M$ of a given size is contained in a model which in turn is contained in $M$. The formalization of this notion begins with the concept of a completion.

5.3 Completions Let $M \in \mathrm{DB}(\mathbf{D})$. A completion of $M$ is any $N \in \operatorname{LDB}(\mathbf{D})$ which contains $M$. There are two important families of completions of $M$, which are detailed below.

(a) The full set of completions of $M$ is $\operatorname{FullCompl}_{\mathbf{D}}(M)=\{N \in \operatorname{LDB}(\mathbf{D}) \mid M \subseteq N\}$.

(b) The set of minimal completions of $M$ is $\operatorname{MinCompl}_{\mathbf{D}}(M)=\left\{S \in \operatorname{FullCompl}_{\mathbf{D}}(M) \mid(\forall T \in\right.$ FullCompl $\mathbf{D}(M))((T \subseteq S) \Rightarrow(T=S))\}$. A minimal completion of $M$ is thus a completion which does not contain any proper subset which is itself a completion of $M$.

Observe that $M \in \operatorname{LDB}(\mathbf{D})$ iff $\operatorname{MinCompl}_{\mathbf{D}}(M)=\{M\}$.

Because a schema with the $k$-submodel property has the unrestricted submodel property as well, it follows that the only possibilities for the set of minimal completions of $M \in \mathrm{DB}(\mathbf{D})$ in that case are $\{M\}$ (in which case $M$ is already in $\operatorname{LDB}(\mathbf{D})$ ) and $\emptyset$ (in which case no extension of $M$ is in $\operatorname{LDB}(\mathbf{D})$ ).

$5.4\left(k_{1}, k_{2}\right)$-boundedness Clearly, the process of completion involves an increase in size of the database. The notion of $\left(k_{1}, k_{2}\right)$-boundedness characterizes this growth locally; that is, for models of size no more than $k_{1}$. Informally, the $\left(k_{1}, k_{2}\right)$-boundedness property states that for any $M \in \operatorname{DB}(\mathbf{D})$ with $\operatorname{Card}(M) \leq k_{1}$, the size of any minimal completion is no more than $k_{2}$. It is necessary to be a bit careful here. Even though all databases are taken to be fi nite, there may be no bound on the size of completions of databases of size $k_{1}$. To model this possibility, let $\overline{\mathbb{N}}$ denote the set consisting of the natural numbers, together with a special element which will be denoted by $\infty$. The $\left(k_{1}, \infty\right)$ boundedness property then provides no information on the maximum size of completions of databases 
of size at most $k_{1}$. The symbol $\infty$ will be used rather loosely, at least to the extent that it may be compared to any natural number; i.e., $m<\infty$ for any $m \in \mathbb{N}$. The formal defi nition is as follows.

(a) For $k_{1} \in \mathbb{N}, k_{2} \in \overline{\mathbb{N}}$, the schema $\mathbf{D}$ has the $\left(k_{1}, k_{2}\right)$-boundedness property if for every $M \in \mathrm{DB}(\mathbf{D})$ with $\operatorname{Card}(M) \leq k_{1}$, every $N \in \operatorname{MinCompl}_{\mathbf{D}}(M)$ has the property that $\operatorname{Card}(N) \leq k_{2}$.

As a specifi c example, a universal relational schema constrained by FDs (or even EGDs) and a single join dependency has the $(2,4)$-boundedness property.

5.5 The $k_{1}$-premodel property The notion of a $k$-premodel and the $k$-premodel property generalize the notions of $k$-model and the $k$-submodel property to the context in which completions can add new elements. A $k$-premodel contains a completion of each of its subsets of size at most $k$, with a schema having the $k$-premodel property iff its legal databases are characterized by $k$-premodels.

More formally, Let $k \in \mathbb{N}$.

(a) A $k$-premodel of $\mathbf{D}$ is an $M \in \mathrm{DB}(\mathbf{D})$ with the property that for every $N \subseteq M$ with $\operatorname{Card}(N) \leq k$, there is a $P \in \operatorname{MinCompl}_{\mathbf{D}}(N)$ with $P \subseteq M$.

(b) The schema $\mathbf{D}$ has the $k$-premodel property if for every $M \in \mathrm{DB}(\mathbf{D})$, the condition $M \in \operatorname{LDB}(\mathbf{D})$ holds iff $M$ is a $k$-premodel of $\mathbf{D}$.

5.6 Relative $\left(k_{1}, k_{2}\right)$-boundedness and relative $k_{1}$-premodels In the framework of [Heg04b], it is always the case that the join dependency underlying the decomposition of the base schema $\mathbf{D}$ into meet-complementary views is generated by the other dependencies, which are in turn embeddable into the component views. In that case, every join-compatible pair of legal view states gives rise to a unique state of the main schema; in the terminology of 4.6(e), the main view has implicit join completion. In the more general framework developed here, this need not be the case. Rather, the join dependency may be specifi ed separately, as elaborated in 4.6. This dependency can force a database in the main schema to be much larger than the sum of the sizes of its components under the decomposition, giving an artifi cially high measure of how large completions can become. Thus, when looking at some completion $N$ of an $M \in \mathrm{DB}(\mathbf{D})$, it is advantageous to separate the increase in size due to the constraints which embed in the view from that caused by the join dependency governing the decomposition. The key is to ask not how large $N$ can be, but rather how large some $N^{\prime} \in \operatorname{DB}(\mathbf{D})$ which completes to $N$ upon applying the join dependency can be. The formalization of these ideas, for both the $k$-submodel and the $k$-premodel contexts, is as follows. Let $k \in \mathbb{N}$, and assume that $\left\{\Gamma_{1}, \Gamma_{2}\right\}$ forms a meet-complementary pair of PF-views of $\mathbf{D}$, and that $\mathbf{D}$ uses join reconstruction from $\left\{\Gamma_{1}, \Gamma_{2}\right\}$ (See 4.6(c)).

(a) $M \in \mathrm{DB}(\mathbf{D})$ is a $\left\langle\Gamma_{1} ; \Gamma_{2}\right\rangle$-relative $k$-premodel of $\mathbf{D}$ if for every $N \subseteq M$ with $\operatorname{Card}(N) \leq k$, there is a $P \subseteq M$ with JoinCompl $\Gamma_{\left.\Gamma_{1} ; \Gamma_{2}\right\rangle}(P) \in \operatorname{MinCompl}_{\mathbf{D}}(N)$.

(b) The schema $\mathbf{D}$ has the $\left\langle\Gamma_{1} ; \Gamma_{2}\right\rangle$-relative k-premodel property if for every $M \in \mathrm{DB}(\mathbf{D})$, the condition $M \in \operatorname{PLDB}\left(\left\langle\overline{\mathbf{D}} ;\left\{\Gamma_{1}, \Gamma_{2}\right\}\right\rangle\right)$ holds iff $M$ is a $\left\langle\Gamma_{1} ; \Gamma_{2}\right\rangle$-relative $k$-premodel of $\mathbf{D}$.

(a') $M \in \mathrm{DB}(\mathbf{D})$ is a $\left\langle\Gamma_{1} ; \Gamma_{2}\right\rangle$-relative $k$-model of $\mathbf{D}$ if every $N \subseteq M$ with $\operatorname{Card}(N) \leq k$ is in $\operatorname{PLDB}\left(\left\langle\overline{\mathbf{D}} ;\left\{\Gamma_{1}, \Gamma_{2}\right\}\right\rangle\right)$.

$\left(\mathrm{b}^{\prime}\right)$ The schema $\mathbf{D}$ has the $\left\langle\Gamma_{1} ; \Gamma_{2}\right\rangle$-relative $k$-submodel property if for every $M \in \mathrm{DB}(\mathbf{D})$, the condition $M \in \operatorname{PLDB}\left(\left\langle\overline{\mathbf{D}} ;\left\{\Gamma_{1}, \Gamma_{2}\right\}\right\rangle\right)$ holds iff $M$ is a $\left\langle\Gamma_{1} ; \Gamma_{2}\right\rangle$-relative $k$-model of $\mathbf{D}$. 
(c) For $k_{1} \in \mathbb{N}, k_{2} \in \overline{\mathbb{N}}$, the schema $\mathbf{D}$ has the $\left\langle\Gamma_{1} ; \Gamma_{2}\right\rangle$-relative $\left(k_{1}, k_{2}\right)$-boundedness property if for every $M \in \mathrm{DB}(\mathbf{D})$ with $\operatorname{Card}(M) \leq k_{1}$ and every $N \in \operatorname{MinCompl}_{\mathbf{D}}(M)$, there is a $P \subseteq M$ with JoinCompl $\operatorname{L\Gamma }_{\left\langle\Gamma_{1} ; \Gamma_{2}\right\rangle}(P)=N$ and $\operatorname{Card}(P) \leq k_{2}$.

5.7 Rules and their containment semantics Characterization of the $k$-premodel and $k$-submodel properties is facilitated greatly through the use of rules on a schema. Informally, a rule on $\mathbf{D}$ states that if certain elements are present, then so too are others.

(a) A rule on $\mathbf{D}$ is a pair $\alpha=(\operatorname{Antc}(\alpha), \operatorname{Cnsq}(\alpha))$ in which $\operatorname{Antc}(\alpha) \in \mathrm{DB}(\mathbf{D})$ and $\operatorname{Cnsq}(\alpha) \subseteq \mathrm{DB}(\mathbf{D})$, with $\operatorname{Antc}(\alpha) \subseteq M$ for each $M \in \mathrm{Cnsq}(\alpha)$. Antc $(\alpha)$ is called the antecedent of $\alpha$, and $\operatorname{Cnsq}(\alpha)$ its set of consequents.

(b) The rule $\alpha$ is called a denial rule if $\operatorname{Cnsq}(\alpha)=\emptyset$, and a generator rule if $\operatorname{Cnsq}(\alpha) \neq \emptyset$.

Roughly, denial rules correspond to constraints such as EGDs, while generator rules correspond to constraints such as TGDs. See 5.9-5.12 below for examples and more details.

(c) Rules(D) (resp. GeneratorRules(D), resp. DenialRules $(\mathbf{D})$ ) denotes the set of all rules (resp. generator rules, resp. denial rules) on $\mathbf{D}$.

Informally, the containment semantics of a rule simply state that a structure which contains its antecedent (as a subset) must also contain one of its consequents.

(d) The containment semantics of rules are defi ned as follows.

(i) For $\alpha \in \operatorname{Rules}(\mathbf{D})$ and $M \in \operatorname{DB}(\mathbf{D}), M \in \operatorname{Mod}(\alpha)$ iff $\operatorname{Antc}(\alpha) \subseteq M$ implies that there is an $N \in \operatorname{Cnsq}(\alpha)$ with $N \subseteq M$.

(ii) For $\mathcal{S} \subseteq \operatorname{Rules}(\mathbf{D})$ and $M \in \operatorname{DB}(\mathbf{D}), M \in \operatorname{Mod}(\mathcal{S})$ iff $M \in \operatorname{Mod}(\alpha)$ for each $\alpha \in \mathcal{S}$.

Since containment semantics will be used exclusively in this paper, the notation $\operatorname{Mod}(-)$ will not result in any ambiguity.

(g) The set of rule constraints of $\mathbf{D}$ is $\operatorname{RuleConstr}(\mathbf{D})=\{\alpha \in \operatorname{Rules}(\mathbf{D}) \mid(\forall M \in \operatorname{LDB}(\mathbf{D}))(M \in$ $\operatorname{Mod}(\alpha))\}$.

5.8 The completion rules of a schema The rules which form the foundation of the results developed here are based upon the notion of minimal completion, as defi ned in 5.3. They are formalized as follows.

(a) Let $M \in \mathrm{DB}(\mathbf{D})$. The minimal-completion rule of $M$ is defi ned to be $\operatorname{MinComplRul} \phi_{\mathbf{D}}(M)=$ $\left(M\right.$, MinCompl $\left._{\mathbf{D}}(M)\right)$. In other words, $\quad \operatorname{Antc}\left(\operatorname{MinComplRule}_{\mathbf{D}}(M)\right)=M$, and Cnsq(MinComplRule $\mathbf{D}(M))=\operatorname{MinCompl}_{\mathbf{D}}(M)$.

(b) Defi ne MinComplRules(D) $=\left\{\operatorname{MinComplRul} \mathbf{q}_{\mathbf{D}}(M) \mid M \in \mathrm{DB}(\mathbf{D})\right\}$.

(c) Let $k \in \mathbb{N}$. Defi ne MinComplRules ${ }^{k}(\mathbf{D})=\left\{\operatorname{MinComplRule}_{\mathbf{D}}(M) \mid(M \in \mathrm{DB}(\mathbf{D})) \wedge(\operatorname{Card}(M) \leq\right.$ $k)\}$.

Although the defi nition of schema semantics via minimal-completion rules has some similarities to the use of classical constraints, there are a number of key differences. In the next four examples, some of these are highlighted. 
5.9 Example - Rule semantics with functional dependencies Let $\mathbf{E}_{5}$ be the relational schema with the single relation $R[A B C D]$, constrained by the FDs $\{A \rightarrow B, C \rightarrow D\}$. It should fi rst of all be noted that rules, as defi ned here, do not support quantifi cation. Thus, it not possible to express the following fi rst-order representation of the FD $A \rightarrow B$ as a single minimal completion rule.

$$
\left(\forall v_{1}^{A}\right)\left(\forall v_{1}^{B}\right)\left(\forall v_{2}^{B}\right)\left(\forall v_{1}^{C}\right)\left(\forall v_{2}^{C}\right)\left(\forall v_{1}^{D}\right)\left(\forall v_{2}^{D}\right)\left(\left(R\left(v_{1}^{A}, v_{1}^{B}, v_{1}^{C}, v_{1}^{C}\right) \wedge R\left(v_{1}^{A}, v_{2}^{B}, v_{2}^{C}, v_{2}^{C}\right) \wedge\left(v_{1}^{B} \neq v_{2}^{B}\right)\right) \Rightarrow \text { false }\right)
$$

Rather, it is necessary to express each ground instance of the formula (i.e., each instance with the variables bound to specifi c constants) as a distinct rule. A generic logical ground instance for the above expression is

$$
\left(\left(R\left(\mathrm{a}_{1}, \mathrm{~b}_{1}, \mathrm{c}_{1}, \mathrm{~d}_{1}\right) \wedge R\left(\mathrm{a}_{1}, \mathrm{~b}_{2}, \mathrm{c}_{2}, \mathrm{~d}_{2}\right)\right) \Rightarrow \text { false }\right)
$$

with $\mathrm{a}_{1}$, as well as the $\mathrm{b}_{i}$ 's, $\mathrm{c}_{i}$ 's, and $\mathrm{d}_{i}$ 's, values from the appropriate domains, and $\mathrm{b}_{1} \neq \mathrm{b}_{2}$. The corresponding minimal-completion (denial) rule is

$$
\left(\alpha_{A \rightarrow B}^{\mathbf{E}_{5}}\right) \quad\left(\left\{\left(\mathrm{a}_{1}, \mathrm{~b}_{1}, \mathrm{c}_{1}, \mathrm{~d}_{1}\right),\left(\mathrm{a}_{1}, \mathrm{~b}_{2}, \mathrm{c}_{2}, \mathrm{~d}_{2}\right)\right\}, \emptyset\right)
$$

Similarly, the FD $C \rightarrow D$ is represented by denial rules of the form

$$
\left(\alpha_{C \rightarrow D}^{\mathbf{E}_{5}}\right) \quad\left(\left\{\left(\mathrm{a}_{1}, \mathrm{~b}_{1}, \mathrm{c}_{1}, \mathrm{~d}_{1}\right),\left(\mathrm{a}_{2}, \mathrm{~b}_{2}, \mathrm{c}_{1}, \mathrm{~d}_{2}\right)\right\}, \emptyset\right)
$$

in which $\mathrm{d}_{1} \neq \mathrm{d}_{2}$, but the other elements may nor may not be equal. All other ordered pairs of tuples result in (trivial) normal rules. That is, rules of the form

$$
\left(\left\{\left(\mathrm{a}_{1}, \mathrm{~b}_{1}, \mathrm{c}_{1}, \mathrm{~d}_{1}\right),\left(\mathrm{a}_{2}, \mathrm{~b}_{2}, \mathrm{c}_{2}, \mathrm{~d}_{2}\right)\right\},\left\{\left\{\left(\mathrm{a}_{1}, \mathrm{~b}_{1}, \mathrm{c}_{1}, \mathrm{~d}_{1}\right),\left(\mathrm{a}_{2}, \mathrm{~b}_{2}, \mathrm{c}_{2}, \mathrm{~d}_{2}\right)\right\}\right\}\right)
$$

in which $\mathrm{a}_{1} \neq \mathrm{a}_{2}$ and $\mathrm{c}_{1} \neq \mathrm{c}_{2}$ are always in $\operatorname{RuleConstr}\left(\mathbf{E}_{4}\right)$. However, these not need be included explicitly in a constraint set for this schema; it is suffi cient that rules of the form $\alpha_{A \rightarrow B}^{\mathbf{E}_{5}}$ and $\alpha_{C \rightarrow D}^{\mathbf{E}_{5}}$ be included. In general, identity rules; that is, rules $\alpha$ for which $\operatorname{Cnsq}(\alpha)=\{\operatorname{Antc}(\alpha)\}$, never need be included in the defi nition of minimal-completion semantics, since the containment semantics of 5.7(d) takes them to be true by default, unless overridden by other rules.

5.10 Example - Rule semantics with a join dependency $\quad$ Next, let $\mathbf{E}_{6}$ be the relational schema with the single relation $R[A B C D]$, constrained by the FD $A \rightarrow B$ and the join dependency $\bowtie[A B C, C D]$. The rule $\alpha_{A \rightarrow B}^{\mathbf{E}_{6}}$, identifi ed above, applies to this schema as well. The join dependency $\bowtie[A B C, C D]$ is represented in $\mathbf{E}_{6}$ by the set of all rules of the form

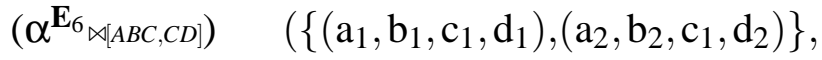

$$
\begin{aligned}
& \left.\left\{\left\{\left(a_{1}, b_{1}, c_{1}, d_{1}\right),\left(a_{2}, b_{2}, c_{1}, d_{2}\right),\left(a_{1}, b_{1}, c_{1}, d_{2}\right),\left(a_{2}, b_{2}, c_{1}, d_{1}\right)\right\}\right\}\right)
\end{aligned}
$$

with either $\mathrm{a}_{1} \neq \mathrm{a}_{2}$ or else $\mathrm{b}_{1}=\mathrm{b}_{2}$. Note that this rule expresses the JD $\bowtie[A B C, C D]$ only in the context of tuples which also satisfy the FD $A \rightarrow B$. It is not possible to drop the conditions that either $a_{1} \neq a_{2}$ or else $b_{1}=b_{2}$. Minimal-completion rules, unlike classical integrity constraints, must have consequents whose members satisfy all of the constraints on the schema. On the other hand, if the FD $A \rightarrow B$ on $\mathbf{E}_{6}$ is dropped, so that the JD is its only constraint, then instances of the above rule with both $\mathrm{a}_{1}=\mathrm{a}_{2}$ and $\mathrm{b}_{1} \neq \mathrm{b}_{2}$ would apply.

Note also that this schema has the $(2,4)$-boundedness property. 
5.11 Example - Rule semantics with multiple generating dependencies A third example illustrates this integration of integrity constraints within rules more saliently. Let $\mathbf{E}_{7}$ have three binary relation symbols $R[A B], S[A B]$, and $T[A B]$, and assume further that $\operatorname{Dom}(A)=\operatorname{Dom}(B)$. Informally, the constraints on $\mathbf{E}_{7}$ state that $S$ contains $R$, as well as the composition of $R$ with itself, and $T$ contains $S$, as well as the composition of $S$ with itself. More formally, these constraints are expressed by the following logical formulas.

$$
\begin{aligned}
&\left(\forall v_{1}^{A}\right)\left(\forall v_{1}^{B}\right)\left(R\left(v_{1}^{A}, v_{1}^{B}\right) \Rightarrow S\left(v_{1}^{A}, v_{1}^{B}\right)\right) \\
&\left(\forall v_{1}^{A}\right)\left(\forall v_{1}^{B}\right)\left(S\left(v_{1}^{A}, v_{1}^{B}\right) \Rightarrow T\left(v_{1}^{A}, v_{1}^{B}\right)\right) \\
&\left(\forall v_{1}^{A}\right)\left(\forall v_{2}^{A}\right)\left(\forall v_{1}^{B}\right)\left(\forall v_{2}^{B}\right)\left(R\left(v_{1}^{A}, v_{1}^{B}\right) \wedge R\left(v_{2}^{A}, v_{2}^{B}\right) \wedge\left(v_{1}^{B}=v_{2}^{A}\right) \Rightarrow S\left(v_{1}^{A}, v_{2}^{B}\right)\right) \\
&\left(\forall v_{1}^{A}\right)\left(\forall v_{2}^{A}\right)\left(\forall v_{1}^{B}\right)\left(\forall v_{2}^{B}\right)\left(S\left(v_{1}^{A}, v_{1}^{B}\right) \wedge S\left(v_{2}^{A}, v_{2}^{B}\right) \wedge\left(v_{1}^{B}=v_{2}^{A}\right) \Rightarrow T\left(v_{1}^{A}, v_{2}^{B}\right)\right)
\end{aligned}
$$

The minimal-completion rules of $\mathbf{E}_{7}$ must embody all of these constraints simultaneously. For single tuples, the rules take on the following forms. (Since tuples may now come from one of several relations, they must be tagged with the name of the relation of origin.)

$$
\begin{array}{ll}
\left(\alpha_{1}^{\mathbf{E}_{7}}\right) & \left(\left\{R\left(\mathrm{a}_{1}, \mathrm{~b}_{1}\right)\right\},\left\{\left\{R\left(\mathrm{a}_{1}, \mathrm{~b}_{1}\right), S\left(\mathrm{a}_{1}, \mathrm{~b}_{1}\right), T\left(\mathrm{a}_{1}, \mathrm{~b}_{1}\right)\right\}\right\}\right) \\
\left(\alpha_{2}^{\mathbf{E}_{7}}\right) & \left(\left\{S\left(\mathrm{a}_{1}, \mathrm{~b}_{1}\right)\right\},\left\{\left\{S\left(\mathrm{a}_{1}, \mathrm{~b}_{1}\right), T\left(\mathrm{a}_{1}, \mathrm{~b}_{1}\right)\right\}\right\}\right) \\
\left(\alpha_{3}^{\mathbf{E}_{7}}\right) & \left(\left\{T\left(\mathrm{a}_{1}, \mathrm{~b}_{1}\right)\right\},\left\{\left\{T\left(\mathrm{a}_{1}, \mathrm{~b}_{1}\right)\right\}\right\}\right)
\end{array}
$$

The third rule is an identity and will not affect the semantics of the total constraint set. Now, for rules with two elements in the antecedent set, it is best to begin with relation $S$. There are two possibilities for an antecedent containing two tuples. First, if $a_{1} \neq b_{2}$, there are rules of the following form.

$\left(\alpha_{4}^{\mathbf{E}_{7}}\right) \quad\left(\left\{S\left(\mathrm{a}_{1}, \mathrm{~b}_{1}\right), S\left(\mathrm{~b}_{1}, \mathrm{~b}_{2}\right)\right\},\left\{\left\{S\left(\mathrm{a}_{1}, \mathrm{~b}_{1}\right), S\left(\mathrm{~b}_{1}, \mathrm{~b}_{2}\right), T\left(\mathrm{a}_{1}, \mathrm{~b}_{1}\right), T\left(\mathrm{~b}_{1}, \mathrm{~b}_{2}\right), T\left(\mathrm{a}_{1}, \mathrm{~b}_{2}\right)\right\}\right\}\right)$

if $\mathrm{a}_{1}=\mathrm{b}_{2}$, the rules take this form.

$\left(\alpha_{5}^{\mathbf{E}_{7}}\right) \quad\left(\left\{S\left(\mathrm{a}_{1}, \mathrm{~b}_{1}\right), S\left(\mathrm{~b}_{1}, \mathrm{a}_{1}\right)\right\},\left\{\left\{S\left(\mathrm{a}_{1}, \mathrm{~b}_{1}\right), S\left(\mathrm{~b}_{1}, \mathrm{a}_{1}\right), T\left(\mathrm{a}_{1}, \mathrm{~b}_{1}\right), T\left(\mathrm{~b}_{1}, \mathrm{a}_{1}\right), T\left(\mathrm{a}_{1}, \mathrm{a}_{1}\right), T\left(\mathrm{~b}_{1}, \mathrm{~b}_{1}\right)\right\}\right\}\right)$

The rules with two antecedent tuples in $R$ are similar, but more complex, since they involve three relation symbols. First, if $\mathrm{a}_{1} \neq \mathrm{b}_{2}$, the rules have the following form.

$$
\begin{aligned}
& \left(\alpha_{6}^{\mathbf{E}_{7}}\right) \\
& \quad\left(\left\{R\left(\mathrm{a}_{1}, \mathrm{~b}_{1}\right), R\left(\mathrm{~b}_{1}, \mathrm{~b}_{2}\right)\right\}\right. \\
& \left.\quad\left\{\left\{R\left(\mathrm{a}_{1}, \mathrm{~b}_{1}\right), R\left(\mathrm{~b}_{1}, \mathrm{~b}_{2}\right), S\left(\mathrm{a}_{1}, \mathrm{~b}_{1}\right), S\left(\mathrm{~b}_{1}, \mathrm{~b}_{2}\right), S\left(\mathrm{a}_{1}, \mathrm{~b}_{2}\right), T\left(\mathrm{a}_{1}, \mathrm{~b}_{1}\right), T\left(\mathrm{~b}_{1}, \mathrm{~b}_{2}\right), T\left(\mathrm{a}_{1}, \mathrm{~b}_{2}\right)\right\}\right\}\right)
\end{aligned}
$$

if $\mathrm{a}_{1}=\mathrm{b}_{2}$, the rules take this form.

$$
\begin{aligned}
\left(\alpha_{7}^{\mathbf{E}_{7}}\right) \quad\left(\left\{R\left(\mathrm{a}_{1}, \mathrm{~b}_{1}\right),\right.\right. & \left.R\left(\mathrm{~b}_{1}, \mathrm{a}_{1}\right)\right\} \\
\{ & \left\{R\left(\mathrm{a}_{1}, \mathrm{~b}_{1}\right), R\left(\mathrm{~b}_{1}, \mathrm{a}_{1}\right), S\left(\mathrm{a}_{1}, \mathrm{~b}_{1}\right), S\left(\mathrm{~b}_{1}, \mathrm{a}_{1}\right), S\left(\mathrm{a}_{1}, \mathrm{a}_{1}\right), S\left(\mathrm{~b}_{1}, \mathrm{~b}_{1}\right),\right. \\
& \left.\left.\left.T\left(\mathrm{a}_{1}, \mathrm{~b}_{1}\right), T\left(\mathrm{~b}_{1}, \mathrm{a}_{1}\right), T\left(\mathrm{a}_{1}, \mathrm{a}_{1}\right), T\left(\mathrm{~b}_{1}, \mathrm{~b}_{1}\right)\right\}\right\}\right)
\end{aligned}
$$

The rules of the form $\alpha_{1}^{\mathbf{E}_{7}}-\alpha_{2}^{\mathbf{E}_{7}}$ and $\alpha_{4}^{\mathbf{E}_{7}}-\alpha_{7}^{\mathbf{E}_{7}}$ suffi ce to identify the legal databases of $\mathbf{E} 7$, in the sense that the models of these rules under the containment semantics of 5.7(d) are suffi cient to identify 
$\operatorname{LDB}\left(\mathbf{E}_{7}\right)$. Since these rules all have antecedents of cardinality no more than two, this schema has the 2-premodel property, although it does not have the 2-submodel property. It also has the $(2,10)$ boundedness property, but it does not have the $(2, k)$-boundedness property for any $k<10$. This is the case even though the logical constraints, as identifi ed above, have a sort of $(2,3)$-boundedness property, since each takes at most two antecedents and generates at most one additional consequent. Thus, the size of the consequents of a generating rule can be much larger than those of a logical constraint.

5.12 Example - rule semantics with an inclusion dependency Let $\mathbf{E}_{8}$ consist of two binary relations $R[A B]$ and $S[A B]$, and suppose that it is constrained by the FD $A \rightarrow B$ on $S$, as well as the inclusion dependency $R[A] \subseteq S[A]$. In other words, $R[A]$ is a foreign key for $S[A]$. The rules which enforce the FD have the following form, for $\mathrm{b}_{1} \neq \mathrm{b}_{2}$.

$\left(\alpha_{1}^{\mathbf{E}_{8}}\right)$

$$
\left(\left\{S\left(\mathrm{a}_{1}, \mathrm{~b}_{1}\right), S\left(\mathrm{a}_{1}, \mathrm{~b}_{2},\right)\right\}, \emptyset\right)
$$

The rules which enforce the foreign-key dependency involve a possibly infi nite disjunction (depending upon the cardinality of the domain $B$ ). They have the following form.

$\left(\alpha_{2}^{\mathbf{E}_{8}}\right) \quad\left(\left\{R\left(\mathrm{a}_{1}, \mathrm{~b}_{1}\right)\right\},\left\{\left\{R\left(\mathrm{a}_{1}, \mathrm{~b}_{1}\right), S\left(\mathrm{a}_{1}, b\right)\right\} \mid b \in \operatorname{Dom}(B)\right\}\right)$

The reason that these examples have been presented is to illustrate the essential difference between minimal-completion rules and ordinary database dependencies. In effect, the right hand side of a minimal-completion rule must always be a collection of legal databases, which may render the representation somewhat more complex. As shall be shown, minimal-completion rules are precisely the kind of representation which is necessary for the study of relative complexity of view axiomatization.

5.13 Projection of rules to views To determine the constraints which a rule on the main schema imposes upon a view, that rule is projected onto the view. The defi nition provided here applies only to minimal-completion rules, but this is not a problem since only such rules are employed in this work. Formally, let $\Gamma=(\mathbf{V}, \gamma)$ be a PF-view of $\mathbf{D}$.

(a) For $\alpha=$ MinComplRule $(M)$, defi ne the projection of $\alpha$ onto $\Gamma$ to be the rule $\gamma(\alpha)=$ MinComplRule $_{\mathbf{V}}(\bar{\gamma}(M))$.

(b) For $\mathcal{S} \subseteq$ Rules $(\mathbf{D}), \gamma(S)=\{\gamma(\alpha) \mid \alpha \in \mathcal{S}\}$.

(c) The set $\mathcal{S} \subseteq \operatorname{Rules}(\mathbf{D})$ is said to define $\Gamma$ semantically if $\operatorname{LDB}(\mathbf{V})=\operatorname{Mod}(\gamma(\mathcal{S}))$.

See the discussion which follows 5.17(c) for an example of a view which is not semantically defi nable.

5.14 Further notational conventions For the rest of this section, the focus will be upon the behavior of minimal-completion rules within the context of a meet-complementary pair. To avoid repeating the context over and over, in addition to $\mathbf{D}=(\operatorname{SynFnd}(\mathbf{D}), \operatorname{LDB}(\mathbf{D}))$ being a PF-schema, unless stated specifi cally to the contrary, it will be assumed that $\Gamma_{1}=\left(\mathbf{V}_{1}, \gamma_{1}\right)$ and $\Gamma_{2}=\left(\mathbf{V}_{2}, \gamma_{2}\right)$ form a meet 
complementary pair of views of $\mathbf{D}$, with meet $\Gamma_{3}=\left(\mathbf{V}_{3}, \gamma_{3}\right)$. It will further be assumed that $\mathbf{D}$ uses join reconstruction from $\left\{\Gamma_{1}, \Gamma_{2}\right\}$.

It is not diffi cult to see that the property of $\left(k_{1}, k_{2}\right)$-boundedness, as well as the property of being a $k$-premodel or $k$-model, is inherited by the views from the main schema. The following two results formalize these facts.

5.15 Observation Let $k_{1} \in \mathbb{N}$ and $k_{2} \in \overline{\mathbb{N}}$, and assume that $\mathbf{D}$ has the $\left\langle\Gamma_{1} ; \Gamma_{2}\right\rangle$-relative $\left(k_{1}, k_{2}\right)$ boundedness property. Then both $\mathbf{V}_{1}$ and $\mathbf{V}_{2}$ have the $\left(k_{1}, k_{2}\right)$-boundedness property.

Proof: Let $i \in\{1,2\}$, let $M \in \mathrm{DB}\left(\mathbf{V}_{i}\right)$ with $\operatorname{Card}(M) \leq k_{1}$, and let $N \in \operatorname{MinCompl}_{\mathbf{V}_{i}}(M)$. Choose $N^{\prime} \in \operatorname{LDB}(\mathbf{D})$ with the property that $\bar{\gamma}_{i}\left(N^{\prime}\right)=N$, and choose $M^{\prime} \subseteq N^{\prime}$ with the property that $\bar{\gamma}_{i}\left(M^{\prime}\right)=M$ and $\operatorname{Card}\left(M^{\prime}\right)=\operatorname{Card}(M)$. Then $N^{\prime} \in \mathrm{FullCompl}_{\mathbf{D}}\left(M^{\prime}\right)$, and so there is an $N^{\prime \prime} \in$ MinCompl $\mathbf{D}\left(M^{\prime}\right)$ with $N^{\prime \prime} \subseteq N^{\prime}$. Since $\mathbf{D}$ has the $\left\langle\Gamma_{1} ; \Gamma_{2}\right\rangle$-relative $\left(k_{1}, k_{2}\right)$-boundedness property and $\operatorname{Card}\left(M^{\prime}\right)=\operatorname{Card}(M) \leq k_{1}$, there is a $P \subseteq N^{\prime \prime}$ with $\operatorname{JoinCompl}_{\left\langle\Gamma_{1} ; \Gamma_{2}\right\rangle}(P)=N^{\prime \prime}$ and $\operatorname{Card}(P) \leq k_{2}$. Now $\bar{\gamma}_{i}(P)=\bar{\gamma}_{i}\left(N^{\prime \prime}\right) \subseteq \bar{\gamma}_{i}\left(N^{\prime}\right)=N$, and since $N \in \operatorname{MinCompl}_{\mathbf{V}_{i}}(M)$, it follows that $\bar{\gamma}_{i}(P)=N$. Furthermore, $\operatorname{Card}(N)=\operatorname{Card}\left(\bar{\gamma}_{i}(P)\right) \leq \operatorname{Card}(P) \leq k_{2}$, which establishes that $\mathbf{V}_{i}$ has the $\left(k_{1}, k_{2}\right)$-boundedness property.

\subsection{Lemma - Projection of $k$-premodels and $k$-models $\quad$ Let $k \in \mathbb{N}$.}

(a) If $M$ is a $\left\langle\Gamma_{1} ; \Gamma_{2}\right\rangle$-relative $k$-premodel of $\mathbf{D}$, then $\bar{\gamma}_{i}(M)$ is a k-premodel of $\mathbf{V}_{i}$ for both $i=1$ and $i=2$.

(b) If $M$ is a $\left\langle\Gamma_{1} ; \Gamma_{2}\right\rangle$-relative $k$-model of $\mathbf{D}$, then $\bar{\gamma}_{i}(M)$ is a $k$-model of $\mathbf{V}_{i}$ for both $i=1$ and $i=2$.

(c) $\gamma\left(\operatorname{MinComplRules}^{k}(\mathbf{D})\right)=\operatorname{MinComplRules}^{k}(\mathbf{V})$.

Proof: Choose $i \in\{1,2\}$. To show (a), let $M$ be a $\left\langle\Gamma_{1} ; \Gamma_{2}\right\rangle$-relative $k$-premodel of $\mathbf{D}$, and let $N^{\prime} \subseteq$ $\bar{\gamma}_{i}(M)$ with $\operatorname{Card}\left(N^{\prime}\right) \leq k$. Choose $N \subseteq M$ with the property that $\bar{\gamma}_{i}(N)=N^{\prime}$ and $\operatorname{Card}(N)=\operatorname{Card}\left(N^{\prime}\right)$. Since $\operatorname{Card}(N)=\operatorname{Card}\left(N^{\prime}\right) \leq k$, there is $P \subseteq M$ with $\operatorname{JoinCompl}_{\left\langle\Gamma_{1} ; \Gamma_{2}\right\rangle}(P) \in \operatorname{MinCompl}_{\mathbf{D}}(N)$. Since $\bar{\gamma}_{i}(P)=\bar{\gamma}_{i}\left(\operatorname{JoinCompl}_{\left\langle\Gamma_{1} ; \Gamma_{2}\right\rangle}(P)\right) \in \operatorname{LDB}\left(\mathbf{V}_{i}\right), \bar{\gamma}_{i}(P) \in \operatorname{FullCompl}_{\mathbf{V}}\left(N^{\prime}\right)$, so there is a $Q \subseteq \bar{\gamma}_{i}(P)$ with $Q \in \operatorname{MinCompl}_{\mathbf{V}}\left(N^{\prime}\right)$. Furthermore, since $P \subseteq M, Q \subseteq \bar{\gamma}_{i}(P) \subseteq \bar{\gamma}_{i}(M)$, whence $\bar{\gamma}_{i}(M)$ is a $k$-model of V.

Part (b) is similar to (a). Just choose $P=N$ and $Q=N^{\prime}$.

To establish part (c), it suffi ces to observe that for every $M \in \mathrm{DB}(\mathbf{V})$, there is an $M \in \mathrm{DB}(\mathbf{D})$ with $\bar{\gamma}(M)=M^{\prime}$ and $\operatorname{Card}(M)=\operatorname{Card}\left(M^{\prime}\right)$.

5.17 Notions of axiomatization for decompositions To address the question of axiomatization of the views in a meet-complementary decomposition, it is fi rst necessary to be very precise about

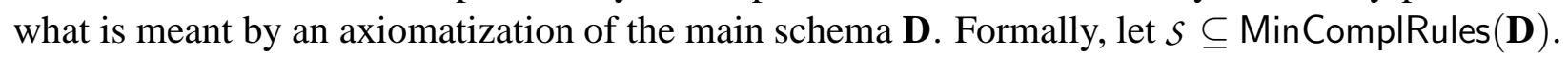

(a) $\mathcal{S}$ is called an $\left\langle\Gamma_{1} ; \Gamma_{2}\right\rangle$-embeddable axiomatization of $\mathbf{D}$ if for every $M \in$ $\operatorname{DB}(\mathbf{D}), M \in \operatorname{PLDB}\left(\left\langle\overline{\mathbf{D}} ;\left\{\Gamma_{1}, \Gamma_{2}\right\}\right\rangle\right)$ iff $\bar{\gamma}_{i}(M) \in \operatorname{Mod}\left(\gamma_{i}(S)\right)$ for both $i=1$ and $i=2$.

(b) $\mathcal{S}$ is called a complete $\left\langle\Gamma_{1} ; \Gamma_{2}\right\rangle$-axiomatization of $\mathbf{D}$ if for every $M \in \operatorname{DB}(\mathbf{D}), \operatorname{Mod}\left(\gamma_{i}(\mathcal{S})\right)=$ $\operatorname{LDB}\left(\mathbf{V}_{i}\right)$ for both $i=1$ and $i=2$. 
The schema $\mathbf{E}_{2}$ of 1.2 illustrates the distinction between these two notions. Let $\mathcal{R}_{2}$ be the set of all minimal-completion rules based upon the FDs in $\mathcal{F}_{2}$, obtained using the techniques outlined in 5.9. It is straightforward to show that $\pi_{A B C D}\left(\mathcal{R}_{2}\right)$ represents $\{A \rightarrow D, B \rightarrow D, C D \rightarrow A\}$, and $\pi_{A B C E}\left(\mathcal{R}_{2}\right)$ represents $\{A \rightarrow E\}$. Thus, $\mathcal{R}_{2}$ is an $\left\{\Pi_{A B C D}, \Pi_{A B C E}\right\}$-embeddable axiomatization of $\mathbf{E}_{2}$.

On the other hand, it cannot be a complete $\left\{\Pi_{A B C D}, \Pi_{A B C E}\right\}$-axiomatization of $\mathbf{E}_{2}$. Indeed, this is the whole point of the example $\mathbf{E}_{2}$. The view $\Pi_{A B C E}$ cannot be axiomatizable by $\pi_{A B C E}\left(R_{2}\right)$. If it were, it would have the 2-submodel property, which means that it would be axiomatizable by FDs. However, as shown in Appendix A, it is not even fi nitely axiomatizable.

It is now possible to establish very sharp results about the decomposition of schemata which have the $k$-submodel property. The decomposition is always $\left\langle\Gamma_{1} ; \Gamma_{2}\right\rangle$-embeddable, and in particular, to verify that a database of the main schema is legal, it suffi ces to verify that the decomposed components are each $k$-models.

5.18 Proposition - View axiomatization via $k$-models Let $k \in \mathbb{N}$ and let $M \in \mathrm{DB}(\mathbf{D})$. Then $M$ is a $\left\langle\Gamma_{1} ; \Gamma_{2}\right\rangle$-relative $k$-model of $\mathbf{D}$ iff $\bar{\gamma}_{i}(M)$ is a $k$-model of $\mathbf{V}_{i}$ for both $i=1$ and $i=2$.

Proof: Let $M \in \mathrm{DB}(\mathbf{D})$ have the property that $\bar{\gamma}_{i}(M)$ is a $k$-model of $\mathbf{V}_{i}$ for both $i=1$ and $i=2$, and let $N \subseteq M$ with $\operatorname{Card}(N) \leq k$. Then $\operatorname{Card}\left(\bar{\gamma}_{i}(N)\right) \leq k$ for both $i=1$ and $i=2$, so $\bar{\gamma}_{i}(N) \in \operatorname{LDB}\left(\mathbf{V}_{i}\right)$. Thus $\left(\hat{\gamma}_{1} \otimes \hat{\gamma}_{2}\right)^{-1}\left(\bar{\gamma}_{1}(N), \bar{\gamma}_{2}(N)\right)=\operatorname{JoinCompl}_{\left\langle\Gamma_{1} ; \Gamma_{2}\right\rangle}(N) \in \operatorname{LDB}(\mathbf{D})$, and so $N \in \operatorname{PLDB}\left(\left\langle\overline{\mathbf{D}} ;\left\{\Gamma_{1}, \Gamma_{2}\right\}\right\rangle\right)$, whence $M$ is a $\left\langle\Gamma_{1} ; \Gamma_{2}\right\rangle$-relative $k$-model of $\mathbf{D}$.

The converse follows immediately from 5.16(b).

5.19 Theorem For any $k \in \overline{\mathbb{N}}$, if $\mathbf{D}$ has the $\left\langle\Gamma_{1} ; \Gamma_{2}\right\rangle$-relative $k$-submodel property, then MinComplRules $^{k}(\mathbf{D})$ is a $\left\langle\Gamma_{1} ; \Gamma_{2}\right\rangle$-embeddable axiomatization of $\mathbf{D}$.

PROOF: The proof follows immediately from 5.18 and 5.16(c).

5.20 Discussion - Interpretation in the classical framework At this point, it is helpful to step back and interpret the above result within the context of the simple example of $\mathbf{E}_{2}$ presented in 1.2. Since $\mathbf{E}_{2}$ is constrained by FDs, it has the 2-submodel property. The above proposition states that to determine whether a relation $r[A B C D E]$ satisfi es the set $\mathcal{F}_{2}$ of FDs, it suffi ces to check whether each subset of $r[A B C E]$ containing at most two tuples and each subset of $r[A B C D]$ containing at most two tuples satisfi es the constraints of the view. However, since the projection of a family of FDs is a family of EGDs ([Fag82, Thm. 6.1]), and since the FDs are precisely the EGDs of degree two (see 6.1) it follows that this reduces to checking the FDs on $\Pi_{A B C E}$ and $\Pi_{A B C D}$. The important point to note is that this characterization does not require that the projected constraints completely axiomatize each view individually. This has already been observed in the discussion of 5.17. The above result shows that the projected constraints, taken together, nonetheless suffi ce to characterize those of the main schema $\mathbf{E}_{2}$.

In the above example, the whole argument may seem rather trivial, since each FD of $\mathcal{F}_{2}$ embeds into one of the views. However, this need not be the case in general. To illustrate, let $\mathbf{E}_{9}$ be the schema with the single relation $R[A B C]$, governed by the FDs $\mathcal{F}_{9}=\{A \rightarrow B, A \rightarrow C, B \rightarrow A, C \rightarrow A\}$, and consider the pair $\left\{\Pi_{A B}, \Pi_{B C}\right\}$ of views. Note that $\mathcal{F}_{9}$ does not embed into the views. While $A \rightarrow B$ and $B \rightarrow A$ embed into $\Pi_{A B}$, the FDs $A \rightarrow C$ and $C \rightarrow A$ embed into neither view, nor are they implied by the pair $\{A \rightarrow B, B \rightarrow A\}$. Therefore, a naïve approach which considers only those FDs 
which embed into the views will not deliver the required constraints. Nonetheless, the decomposition $\left\{\Pi_{A B}, \Pi_{B C}\right\}$ is easily seen to be meet complementary. In the classical theory, the way to prove this is to fi nd a cover of $\mathcal{F}_{9}$ (i.e., a set of equivalent FDs) which does embed into the two views. One such cover is $\mathcal{F}_{9}^{\prime}=\{A \rightarrow B, B \rightarrow C, B \rightarrow A, C \rightarrow B\}$. which is easily seen to be equivalent to $\mathcal{F}_{9}$. The set $\{A \rightarrow B, B \rightarrow A\}$ embeds into $\Pi_{A B}$, while $\{B \rightarrow C, C \rightarrow B\}$ embeds into $\Pi_{B C}$.

At fi rst glance, this might seem to contradict the above results. However, it does not. The reason is, once again, the distinction between classical logical constraints and minimal-completion rules. Consider a generic ground rule for the FD $A \rightarrow C$, with $\mathrm{c}_{1} \neq \mathrm{c}_{2}$.

$\left(\alpha_{A \rightarrow C}^{\mathbf{E}_{9}}\right)$

$$
\left(\left\{\left(a_{1}, b_{1}, c_{1}\right),\left(a_{1}, b_{2}, c_{2}\right)\right\}, \emptyset\right)
$$

If $A \rightarrow C$ were the only constraint in $\mathcal{F}_{9}$, then both projections of this rule (onto $\Pi_{A B}$ and onto $\Pi_{B C}$ ) would be identity rules. However, rules must take into consideration the entire constraint set, so in the context of $\mathcal{F}_{9}$, the projection onto $\Pi_{A B}$ is the denial $\left(\left\{\left(\mathrm{a}_{1}, \mathrm{~b}_{1}\right),\left(\mathrm{a}_{1}, \mathrm{~b}_{2}\right)\right\}, \emptyset\right)$ in the case that $\mathrm{b}_{1} \neq \mathrm{b}_{2}$. On the other hand, if $\mathrm{b}_{1}=\mathrm{b}_{2}$, then the projection onto $\Pi_{B C}$ is the denial $\left(\left\{\left(\mathrm{b}_{1}, \mathrm{c}_{1}\right),\left(\mathrm{b}_{1}, \mathrm{c}_{2}\right)\right\}, \emptyset\right)$, in view of the fact that the FD $B \rightarrow C$ is implied by $\mathcal{F}_{9}$. Thus, while the rule $\alpha_{A \rightarrow C}^{\mathbf{E}_{9}}$ above represents only $A \rightarrow C$, the semantics of projection defi ned in 5.13(b) ensure that the information about the other constraints which must embed into the views is taken into account automatically. The key point of the above result, in the context of denial rules, is that taking this additional information into account does not increase the complexity of the rules. For sets of FDs, this can of course be proved in a more direct fashion, since an embeddable cover of a set of FDs is always as set of FDs. However, for more complex families of constraints, this may not be the case.

5.21 Example - Difficulties in extension to generating rules Based upon the strong result expressed in 5.19 for schemata constrained by denial rules, it is natural to conjecture that a similar result holds in the presence of generating rules. Unfortunately, this is not the case. Indeed, for any $n \in \mathbb{N}$, there is a schema $\mathbf{E}_{10}$ with the 2-premodel property, and a meet complementary pair $\left\{\Omega_{101}, \Omega_{102}\right\}$ of views of that schema with the property that the schema of $\Omega_{102}$ does not have the $k$-premodel property for any $k \leq 2^{n-1}$. The example which illustrates this is quite simple. Let $n \in \mathbb{N}$, and let $A=\left\{a_{i} \mid 1 \leq i \leq 2^{n}-1\right\}$ be an indexed set of elements. Let $b$ be any element not in $A$, and defi ne $S=A \cup\{b\}$. Regard the elements of $A$ as forming a complete binary tree, represented in sequential fashion [HSR98, Sec. 2.2.2]. For each vertex $a_{i}$ with $1 \leq i \leq 2^{n-1}$, the left child is $a_{2 i}$ and the right child is $a_{2 i+1}$. Conversely, the parent of $a_{i}$ for $i>1$ is $a_{\lfloor i / 2\rfloor}$, with $\lfloor i / 2\rfloor$ denoting $n / 2$ rounded down to the nearest integer. The elements of $S$ form the syntactic basis for the PF-schema $\mathbf{E}_{10}$; the elements of $\operatorname{LDB}\left(\mathbf{E}_{10}\right)$ are defi ned to be precisely those subsets of $S$ which satisfy the following constraints.

(i) A parent vertex of the tree defi ned by $A$ is in $M \in \operatorname{LDB}\left(\mathbf{E}_{10}\right)$ iff both of its children are. (Note that one child may be present without the parent.)

(ii) If $a_{1} \in M$, then $b \in M$ as well.

It is easy to see that $\mathbf{E}_{10}$ has the 2-premodel property.

Now, defi ne two views of $\mathbf{E}_{10}$. The view $\Omega_{101}$ has $A$ as the syntactic basis of its schema $\mathbf{E}_{101}$, with the view mapping $M \mapsto M \cap A$. In other words, $\Omega_{101}$ preserves the tree of $A$ but drops $b$. For the view $\Omega_{102}$, defi ne $A^{\prime}=\left\{a_{i} \mid 2^{n-1} \leq i \leq 2^{n}-1\right\}$, and let the syntactic basis of the underlying schema $\mathbf{E}_{102}$ be $A^{\prime} \cup\{b\}$, with the view mapping $M \mapsto M \cap\left(A^{\prime} \cup\{b\}\right)$. In other words, $\Omega_{102}$ preserves $b$ as well as the leaf nodes of the tree formed by $A$, but drops all interior nodes of that tree. It is easy to see 
that $\left\{\Omega_{101}, \Omega_{102}\right\}$ forms a meet-complementary pair. The meet is the view which keeps just $A^{\prime}$; i.e., the leaf nodes of the tree. It follows that for any $M \in \operatorname{LDB}\left(\mathbf{E}_{10}\right), a_{1} \in M$ iff $A^{\prime} \subseteq M$. In light of the constraint identifi ed in (ii) above, the following constraint must hold in $\mathbf{E}_{10}$ and so in $\mathbf{E}_{102}$ as well.

(iii) If every element of $A^{\prime}$ is in $M \in \operatorname{LDB}\left(\mathbf{E}_{102}\right)$, then so too is $b$.

However, this constraint cannot be represented any more succinctly within that view, since the structure of the interior vertices of the tree is not available. There are no other constraints on that view. Clearly, this prevents $\mathbf{E}_{102}$ from having the $k$-premodel property for any $k<2^{n-1}$.

There is a class of decompositions for which such problems do not occur. These views have the property that if two view states match on the meet, then so too do all completions.

\subsection{Meet uniformity of rules}

(a) A set $\mathcal{S} \subseteq \operatorname{Rules}(\mathbf{D})$ is called $\Gamma_{3}$-uniform, or just meet uniform, if whenever $\alpha_{1}, \alpha_{2} \in$ $\mathcal{S} \cap$ GeneratorRules $(\mathbf{D})$ with $\bar{\gamma}_{3}\left(\operatorname{Antc}\left(\alpha_{1}\right)\right)=\bar{\gamma}_{3}\left(\operatorname{Antc}\left(\alpha_{2}\right)\right)$, then for every $M_{1} \in \operatorname{Cnsq}\left(\alpha_{1}\right)$ and $M_{2} \in \operatorname{Cnsq}\left(\alpha_{2}\right), \bar{\gamma}_{3}\left(M_{1}\right)=\bar{\gamma}_{3}\left(M_{2}\right)$.

(b) For $k \in \mathbb{N}$, the schema $\mathbf{D}$ is called $\left\langle k, \Gamma_{3}\right\rangle$-uniform if the set $\operatorname{MinComplRules}^{k}(\mathbf{D})$ is $\Gamma_{3}$-uniform.

Before moving on to the main decomposition result, it is necessary to establish a bound on how large a "basis" or "skeleton" for a join-complete set must be, in terms of the size of its projections to the views of the decomposition.

5.23 Lemma - Representation of join-complete sets Let $M \in \operatorname{JDB}_{\left\langle\Gamma_{1} ; \Gamma_{2}\right\rangle}(\overline{\mathbf{D}})$. Then there is a set $N \subseteq M$ containing at most $\operatorname{Card}\left(\bar{\gamma}_{1}(M)\right)+\operatorname{Card}\left(\bar{\gamma}_{2}(M)\right)$ elements with the property that JoinCompl $_{\left\langle\Gamma_{1} ; \Gamma_{2}\right\rangle}(N)=M$.

PROOF: Begin by partitioning $M$ into three disjoint sets. Let $S_{1}=\left\{a \in M \mid \gamma_{2}^{\sharp}(a)=\eta_{\mathbf{v}_{2}}\right\}, S_{2}=$ $\left\{a \in M \mid \gamma_{1}^{\sharp}(a)=\eta_{\mathbf{v}_{1}}\right\}$, and $S_{3}=\left\{a \in M \mid\left(\gamma_{1}^{\sharp}(a) \in \operatorname{SynFnd}\left(\mathbf{V}_{1}\right)\right)\right.$ and $\left.\left(\gamma_{2}^{\sharp}(a) \in \operatorname{SynFnd}\left(\mathbf{V}_{2}\right)\right)\right\}$. (Recall the defi nition of $\eta_{\mathrm{v}_{i}}$ from 3.2(c)). In other words, $S_{1}$ consists of those elements which project only onto $\Gamma_{1}, S_{2}$ those which project only onto $\Gamma_{2}$, and $S_{3}$ those which project onto both $\Gamma_{1}$ and $\Gamma_{2}$. Next, choose $T_{1} \subseteq S_{1}$ such that $\gamma_{1}^{\sharp}$ is injective on $T_{1}$; that is, $a_{1}, a_{2} \in T_{1}$ and $\gamma_{1}^{\sharp}\left(a_{1}\right)=\gamma_{1}^{\sharp}\left(a_{2}\right)$ implies $a_{1}=a_{2}$. Similarly, choose $T_{2} \subseteq S_{2}$ such that $\gamma_{2}^{\sharp}$ is injective on $T_{2}$. Thus, $\operatorname{Card}\left(T_{i}\right)=\operatorname{Card}\left(\bar{\gamma}_{i}\left(S_{i}\right)\right)$ for both $i=1$ and $i=2$. For any $Q \subseteq S_{3}$, call an element $a \in Q$ redundant for $Q$ if there are $a_{1}, a_{2} \in Q$ with $a_{1} \neq a, a_{2} \neq a$, and with the property that $\gamma_{i}^{\sharp}\left(a_{i}\right)=\gamma_{i}^{\sharp}(a)$ for both $i=1$ and $i=$ 2. Let $T_{3} \subseteq S_{3}$ be obtained from $S_{3}$ by repeatedly removing redundant elements, until there are no more. Clearly $\bar{\gamma}_{i}\left(T_{3}\right)=\bar{\gamma}_{i}\left(S_{3}\right)$ for both $i=1$ and $i=2$. Furthermore, since $T_{3}$ does not contain any redundant elements, $\operatorname{Card}\left(T_{3}\right) \leq \operatorname{Card}\left(\bar{\gamma}_{1}\left(S_{3}\right)\right)+\operatorname{Card}\left(\bar{\gamma}_{2}\left(S_{3}\right)\right)$. Defi ne $N=T_{1} \cup T_{2} \cup T_{3}$. Then $\operatorname{Card}(N)=\operatorname{Card}\left(T_{1}\right)+\operatorname{Card}\left(T_{2}\right)+\operatorname{Card}\left(T_{3}\right) \leq \operatorname{Card}\left(\bar{\gamma}_{1}\left(S_{1}\right)\right)+\operatorname{Card}\left(\bar{\gamma}_{2}\left(S_{2}\right)\right)+\operatorname{Card}\left(\bar{\gamma}_{1}\left(S_{3}\right)\right)+$ $\operatorname{Card}\left(\bar{\gamma}_{2}\left(S_{3}\right)\right) \leq \operatorname{Card}\left(\bar{\gamma}_{1}(M)\right)+\operatorname{Card}\left(\bar{\gamma}_{2}(M)\right)$. Furthermore, for both $i=1$ and $i=2, \bar{\gamma}_{i}(N)=\bar{\gamma}_{i}(M)$, so JoinCompl $\left.\right|_{\left.\Gamma_{1} ; \Gamma_{2}\right\rangle}(N)=M$, completing the proof.

Now, the results of 5.18 and 5.19 may be extended to the case of meet-uniform rules.

5.24 Proposition - View axiomatization via $\left\langle\Gamma_{1} ; \Gamma_{2}\right\rangle$-models Let $k_{1} \in \mathbb{N}, k_{12}, k_{22} \in \overline{\mathbb{N}}$, and assume that $\mathbf{D}$ is $\left\langle k_{1}, \Gamma_{3}\right\rangle$-uniform. 
(a) For any $M \in \mathrm{DB}(\mathbf{D}), M$ is a $\left\langle\Gamma_{1} ; \Gamma_{2}\right\rangle$-relative $k_{1}$-premodel of $\mathbf{D}$ iff $\bar{\gamma}_{i}(M)$ is a $k_{1}$-premodel of $\mathbf{V}_{i}$ for both $i=1$ and $i=2$.

(b) If $\mathbf{V}_{1}$ has the $\left(k_{1}, k_{21}\right)$-boundedness property, and $\mathbf{V}_{2}$ has the $\left(k_{1}, k_{22}\right)$-boundedness property, then $\mathbf{D}$ has the $\left\langle\Gamma_{1} ; \Gamma_{2}\right\rangle$-relative $\left(k_{1}, k_{21}+k_{22}\right)$-boundedness property.

Proof: To show part (a), let $M \in \mathrm{DB}(\mathbf{D})$ have the property that $\bar{\gamma}_{1}(M)$ is a $k_{1}$-premodel of $\mathbf{V}_{1}$ and $\bar{\gamma}_{2}(M)$ is a $k$-premodel of $\mathbf{V}_{2}$, and let $N \subseteq M$ with $\operatorname{Card}(N) \leq k_{1}$. Then for both $i=1$ and $i=2$, $\operatorname{Card}\left(\bar{\gamma}_{i}(N)\right) \leq k_{1}$ as well, so using the fact that $\bar{\gamma}_{i}(M)$ is a $k_{1}$-premodel, choose $P_{i} \in \operatorname{MinCompl}_{\mathbf{V}_{i}}\left(\bar{\gamma}_{i}(N)\right)$ with $P_{i} \subseteq \bar{\gamma}_{i}(M)$ for $i=1$ and $i=2$. Since $\mathbf{D}$ is $\Gamma_{3}$-uniform, it follows that $\overline{\lambda\left\langle\Gamma_{1}, \Gamma_{3}\right\rangle}\left(P_{1}\right)=$ $\overline{\lambda\left\langle\Gamma_{2}, \Gamma_{3}\right\rangle}\left(P_{2}\right)$, so $\left(\bar{\gamma}_{1} \otimes \bar{\gamma}_{2}\right)^{-1}\left(P_{1}, P_{2}\right) \in \operatorname{LDB}(\mathbf{D})$. For notational convenience, defi ne $P=$ $\left(\bar{\gamma}_{1} \otimes \bar{\gamma}_{2}\right)^{-1}\left(P_{1}, P_{2}\right)$. Since $P_{i} \subseteq \bar{\gamma}_{i}(M)$ for both $i=1$ and $i=2$, it follows that $P \subseteq \operatorname{JoinCompl}_{\left\langle\Gamma_{1} ; \Gamma_{2}\right\rangle}(M)$. Hence, $P \in$ FullCompl $_{\mathbf{D}}(N)$. It is furthermore the case that $P \in \operatorname{MinCompl}_{\mathbf{D}}(N)$. Indeed, if $P^{\prime} \in$ $\operatorname{MinCompl}_{\mathbf{D}}(N)$ with $P^{\prime} \subseteq P$, then $\bar{\gamma}_{i}(N) \subseteq \bar{\gamma}_{i}\left(P^{\prime}\right) \subseteq \bar{\gamma}_{i}(P)$ for both $i=1$ and $i=2$, and since $\bar{\gamma}_{i}(P)=$ $P_{i} \in \operatorname{MinCompl}_{\mathbf{V}_{i}}\left(\bar{\gamma}_{i}(N)\right)$, it follows that $\bar{\gamma}_{i}\left(P^{\prime}\right)=\bar{\gamma}_{i}(P)=P_{i}$ for both $i=1$ and $i=2$, whence $P=P^{\prime}$. Finally, set $P^{\prime \prime}=M \cap P$ to obtain $P^{\prime \prime} \subseteq M$ with JoinCompl ${ }_{\left\langle\Gamma_{1} ; \Gamma_{2}\right\rangle}\left(P^{\prime \prime}\right) \in \operatorname{MinCompl}_{\mathbf{D}}(N)$. The converse follows immediately from 5.16(a).

To show part (b), fi rst note that by assumption, $\operatorname{Card}\left(P_{i}\right) \leq k_{2 i}$ for $i=1$ and $i=2$. Then, rather than

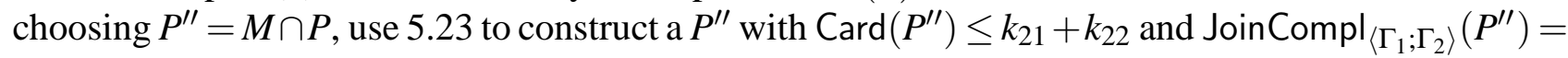
P.

5.25 Theorem For any $k \in \overline{\mathbf{N}}$, if $\mathbf{D}$ is $\Gamma_{3}$-uniform and has the $\left\langle\Gamma_{1} ; \Gamma_{2}\right\rangle$-relative $k$-premodel property, then MinComplRules ${ }^{k}(\mathbf{D})$ is a $\left\langle\Gamma_{1} ; \Gamma_{2}\right\rangle$-embeddable axiomatization of $\mathbf{D}$.

ProOF: The proof follows immediately from 5.24 and 5.16(a)+(c).

5.26 The applicability of meet uniformity The results of 5.24 and 5.25 provide an extension of those of 5.18 and 5.19 in a context of relatively strong independence of the two views comprising the meet-complementary pair. Unfortunately, it fails to supports a level of independence which is adequate in some practical situations. For example, let $\mathbf{E}_{11}$ be the relational schema constrained by the single relation $R[A B C D E]$, together with the JD $\bowtie[A B C, C D E]$ and the two inclusion dependencies $R[A] \subseteq R[B]$ and $R[D] \subseteq R[E]$. It is easy to see that the pair $\left\{\Pi_{A B C}, \Pi_{C D E}\right\}$ forms a meetcomplementary pair, since $R[A] \subseteq R[B]$ embeds in $\Pi_{A B C}$ and $R[D] \subseteq R[E]$ embeds in $\Pi_{C D E}$. Yet, this decomposition is not meet uniform, since the two inclusion dependencies may generate essentially arbitrary values for attribute $C$.

As shown in 5.21, in the presence of generating rules, it is nonetheless necessary to exercise some control over the nature of the meet. What seems to be necessary is a way to distinguish the inconsequential additions to it made by the inclusions dependencies of $\mathbf{E}_{11}$ from the clever encoding of information which is illustrated by the example of 5.21. The development of a theory which addresses this distinction in a general way must be left as a topic for future research. However, as a fi rst step in this direction, in the next section, it is shown that for situations involving the relational model which include $\mathbf{E}_{11}$, it is possible to characterize, in a very useful way, characteristics which a meet-complementary decomposition imposes upon the constraints of the schema.

The results of 5.18 and 5.19, as well as 5.24 and 5.25, extend those of [Heg04b] in a signifi cant way. In that paper, the focus was on the complexity of verifying the correctness of updates on a view. So rather than identifying the complexity of an embedded cover of the constraints of the main 
schema, only the complexity of verifying that a proposed state of the view schema is correct, under the assumption that the state of the complement is already correct, was established (as 4.14 of that paper). Knowledge of the complexity of the embedded cover provides more information. These ideas are formalized in the following, for both the $k$-submodel and $k$-premodel contexts.

5.27 The relative generalized submodel property Suppose that $\Gamma_{1}$ is to be updated with constantcomplement $\Gamma_{2}$. According to [Heg04a, 3.10] (see also the summary in 2.1 of this paper), the allowable updates are precisely those which hold the meet $\Gamma_{1} \wedge \Gamma_{2}=\Gamma_{3}$ constant. Therefore, when performing such an update, it is known that for any proposed new state $M \in \mathrm{DB}\left(\mathbf{V}_{1}\right)$, its projection onto $\Gamma_{3}$ is already legal; i.e., $\overline{\lambda\left\langle\Gamma_{1}, \Gamma_{2}\right\rangle}(M) \in \operatorname{LDB}\left(\mathbf{V}_{3}\right)$. This information may be used to reduce substantially the number constraints which need to be checked; the following defi nitions formalize these concepts.

Let $k \in \mathbb{N}$ and let $i \in\{1,2\}$. The ideas below generalize the those of [Heg04b, 4.13] for $k$ premodels and $k$-submodels.

(a) The database $M \in \mathrm{DB}\left(\mathbf{V}_{i}\right)$ is called $\Gamma_{3}$-legal if $\overline{\lambda\left\langle\Gamma_{i}, \Gamma_{3}\right\rangle}(M) \in \operatorname{LDB}\left(\mathbf{V}_{3}\right)$, and it is called a $\Gamma_{3^{-}}$ relative $k$-premodel (resp. $\Gamma_{3}$-relative $k$-submodel) for $\mathbf{V}_{i}$ if it is both $\Gamma_{3}$-legal and a $k$-premodel (resp. $k$-model) of $\mathbf{V}_{i}$.

(b) The view $\Gamma_{i}=\left(\mathbf{V}_{i}, \gamma_{i}\right)$ has the $\Gamma_{3}$-relative $k$-premodel property (resp. $\Gamma_{3}$-relative $k$-submodel property) if, for every $M \in \mathrm{DB}\left(\mathbf{V}_{i}\right)$, the condition $M \in \operatorname{LDB}\left(\mathbf{V}_{i}\right)$ holds iff $M$ is a generalized $\Gamma_{3}$-relative $k$-premodel (resp. a generalized $\Gamma_{3}$-relative $k$-model) for $\mathbf{V}_{i}$.

The next and fi nal theorem of this section establishes formally that the complexity of testing updates on $\Gamma_{1}$, with $\Gamma_{2}$ held constant, is defi ned by the generalized $\Gamma_{3}$-relative $k$-submodel property on $\mathbf{V}_{1}$, even in the case that the full schema $\mathbf{V}_{2}$ itself has a much higher degree of complexity for its set of constraints.

\subsection{Theorem - Relative complexity for view updates under closed strategies Let $k \in \mathbb{N}$.}

(a) If $\mathbf{D}$ has the $\left\langle\Gamma_{1} ; \Gamma_{2}\right\rangle$-relative $k$-submodel property, then both $\Gamma_{1}$ and $\Gamma_{2}$ have the $\Gamma_{3}$-relative $k$-submodel property.

(b) If $\mathbf{D}$ is $\Gamma_{3}$ uniform and has the $\left\langle\Gamma_{1} ; \Gamma_{2}\right\rangle$-relative $k$-premodel property, then both $\Gamma_{1}$ and $\Gamma_{2}$ have the $\Gamma_{3}$-relative $k$-premodel property.

PROOF: The proof follows directly from 5.19 and 5.25.

\section{An Application within the Relational Framework}

In the classical relational theory - as well as in practice - the two most important types of dependencies are the functional dependencies and the inclusion dependencies. The latter are central to the modelling of foreign-key constraints, which are a part of standard SQL and virtually all modern database-management systems. While the theory of the previous section provides very strong results for schemata constrained by functional dependencies (since they satisfy the 2-submodel property), it does not provide any results for inclusion dependencies, since they are neither universal (and so do 
not satisfy the $k$-submodel property for any $k$ ), nor do they defi ne meet-uniform minimal completions (and so 5.25 does not include schemata which are constrained by them).

Given the importance of these families of constraints, it is essential for any theory of decomposition to address schemata and decompositions in which they occur. In this section, the question of the complexity of view axiomatization is addressed for multi-relation schemata constrained by EGDs and an important subset of the implicational dependencies called fanout-free unary inclusion dependencies (fanout-free UINDs). This class of inclusion dependencies is nonetheless quite powerful; in particular, it is suffi cient to model foreign-key constraints. Using ideas reported in [CKV90], which show that EGDs and UINDs essentially decouple from one another in terms of inference, it is established that in the context of a meet-complementary decomposition defi ned by projections of the component relations, if the main schema is constrained by a combination of EGDs of degree at most $k$ and fanout-free UINDs, then to verify that a candidate database of the main schema is legal, it suffi ces to check that the decomposed components each satisfy all embedded EGDs of degree at most $k$, as well as all embedded UINDs. In particular, if the EGDs are FDs, then it suffi ces to check that the both components of the decomposition satisfy all embedded FDs as well as all embedded UINDs.

6.1 EGDs and UINDs The topic of dependencies on relational databases has been studied extensively, if not exhaustively [Tha91]. Therefore, the discussion here is limited to establishing notation and nonstandard conventions.

It is assumed that there is a fi nite set $\mathbf{U}$ of attributes, as well as a fi nite set of relation symbols $\left\{R_{i} \mid 1 \leq i \leq n\right\}$. With each relation symbol is associated a $\mathbf{U}_{i} \subseteq \mathbf{U}$, with $\mathbf{U}_{i} \cap \mathbf{U}_{j}=\emptyset$ for $i \neq j$ and $\cup_{i=1}^{n} \mathbf{U}_{i}=\mathbf{U}$. There is also an infi nite set $\operatorname{Dom}(\mathbf{U})$ called the universe of domain values. Following standard conventions, a tuple over $R_{i}$ is a function $t: \mathbf{U}_{i} \rightarrow \operatorname{Dom}(\mathbf{U})$, and a relation for $R_{i}$ is a fi nite set of tuples over $R_{i}$. A database is just a set of relations, one for each $R_{i}$. Sets of attributes are often written linearly, so $R[A B C]$ is shorthand for $R[\{A, B, C\}]$.

The universal dependencies (i.e., those dependencies which are representable using logical formulas involving only universal quantifi ers) which are used in this work are called full implicational dependencies (FIDs) [CKV90], or sometimes just implicational dependencies (IDs) [Fag82] [Hul84]. These include the functional dependencies (FDs) and join dependencies (JDs) which are ubiquitous in the relational theory. The three references just cited all give readable summaries of these constraints, so there is no need to repeat them here. Suffi ce to say that they are always unirelational (that is, they apply to only one relation), and they are always typed; that is, they can make comparisons of tuples only between entries in the same column. Because they are used fundamentally in the results developed in this section, it is perhaps appropriate to say a bit more about the equality-generating dependencies (EGDS) [PDGV89, Sec. 3.6], [AHV95, 10.1]. Such dependencies take the following general form:

$$
(\forall \cdot))\left(\left(t_{1} \wedge t_{2} \wedge \ldots \wedge t_{n}\right) \Rightarrow \varepsilon\right)
$$

The terms on the left-hand side are (constant-free) atoms in the language of the relations, while the right-hand side is an equality of two of the variables from the left-hand side. As an example, here is the formula for the functional dependency $A \rightarrow B$ on $R[A B C]$.

$$
R\left(x_{1}, y_{1}, z_{1}\right) \wedge R\left(x_{1}, y_{2}, z_{2}\right) \Rightarrow\left(y_{1}=y_{2}\right)
$$

The degree of an EGD is the number of atoms which occur on the left-hand side of the defi ning formula. The FDs are precisely the EGDs which can be expressed with degree two, and so a relational 
schema constrained by EGDs has the 2-submodel property iff it has a basis consisting of FDs. Although EGDs which are not FDs exist and are not diffi cult to construct, it is not clear that they have any practical use. Nonetheless, since the theory developed here supports them with no additional effort, and since the inclusion of EGDs in fact shows more clearly how the complexity patterns behave, they are retained in their full generality.

To follow the constructions below (particularly in 6.16 and 6.19), it is essential to understand in detail what is meant by a typed constraint. By way of example, the following constraint is not typed, because it compares values in two different columns,

$$
R\left(x_{1}, y_{1}, z_{1}\right) \wedge R\left(y_{2}, x_{1}, z_{2}\right) \Rightarrow\left(y_{1}=y_{2}\right)
$$

and the following is not typed because it equates values in two different columns.

$$
R\left(x_{1}, y_{1}, z_{1}\right) \wedge R\left(x_{1}, y_{2}, z_{2}\right) \Rightarrow\left(y_{1}=z_{2}\right)
$$

The family of inclusion dependencies (INDs) is neither typed nor universal. In its most general form, for $\mathbf{Y}_{i} \subseteq \mathbf{U}_{i}$ and $\mathbf{Y}_{j} \subseteq \mathbf{U}_{j}$, the inclusion dependency $R_{i}\left[\mathbf{Y}_{i}\right] \subseteq R_{j}\left[\mathbf{Y}_{j}\right]$ stipulates that for every tuple $r$ in the instance of $R_{i}$, there is a tuple $s$ in the instance of $R_{j}$ for which $r\left[\mathbf{Y}_{i}\right]=s\left[\mathbf{Y}_{j}\right]$. In their general form, INDs are very complex. For example, it is known that the inference problem for INDs and FDs together is undecidable [CV85]. For this reason, various subfamilies of the INDs have been studied as well. For this work, the most important is the unary INDs (UINDs), in which both $\mathbf{Y}_{i}$ and $\mathbf{Y}_{j}$ are restricted to contain just one attribute. For UINDs, various associated inference problems are not only decidable, but of reasonable complexity as well. Furthermore, and central to this paper, in the associated inference problem, the effects of the INDs and other dependencies (IDs, etc.) can be essentially "decoupled" [CKV90]. Since each attribute occurs in exactly one relation, the UID $R_{i}\left[\mathbf{Y}_{i}\right] \subseteq R_{j}\left[\mathbf{Y}_{j}\right]$ may be unambiguously abbreviated to just $\mathbf{Y}_{i} \subseteq \mathbf{Y}_{j}$. However, since the symbol " $\subseteq$ " is already overloaded mathematically, the alternate notation $\mathbf{Y}_{i} \sqsubseteq \mathbf{Y}_{j}$ using the squared inclusion symbol will be used in the presentation which follows. (More precisely, since $\mathbf{Y}_{i}=\left\{A_{i}\right\}$ and $\mathbf{Y}_{j}=\left\{A_{j}\right\}$ are singletons in the case of UINDs, the resulting notation is just $A_{i} \sqsubseteq A_{j}$.) Occasionally, the notation $A_{j} \sqsupseteq A_{i}$ will be used; it has the same meaning as $A_{i} \sqsubseteq A_{j}$. Similarly, $A_{i} \sqsubseteq A_{j} \sqsubseteq A_{k}$ means that both $A_{i} \sqsubseteq A_{j}$ and $A_{j} \sqsubseteq A_{k}$ hold.

There is one further point of framework to be made. In much of the classical theory of dependencies, it is further assumed that $\operatorname{Dom}(\mathbf{U})$ is partitioned into disjoint sets $\{\operatorname{Dom}(A) \mid A \in \mathbf{U}\}$, with a tuple entry for attribute $A$ restricted to take values from $\operatorname{Dom}(A)$. In the context of typed dependencies, this makes complete sense. However, since inclusion dependencies are not typed, this convention cannot be used here. Rather, the same domain value must be permitted in different columns, both of the same relation and of different relations. All that can be said is that $\operatorname{Dom}(A) \subseteq \operatorname{Dom}(\mathbf{U})$ for each $A \in \mathbf{U}$.

Finally, it is appropriate to recall a point of notation. If $\Phi$ is a set of constraints of some type T (e.g., FIDs, UINDs, EGDs, FDs, etc.), then $\Phi^{+}$is used to denote the set of all constraints of that same class which are consequences of those in $\Phi$. For the purposes of this paper, this will always mean finite implication. See 6.7 below for more details.

6.2 Projections of EGDs The theory developed here is based upon views of relational schemata which are defi ned by projections of relations onto subsets of their attributes. The problem of characterizing the dependencies on a view is called the implied constraint problem [JAK82], of which there are two dimensions. On the one hand, there is the aspect of complexity; as illustrated by the example 
of 1.1, the constraints on the view may be far more complex than those on the main schema. On the other hand, there is the aspect of form. Again, the example of 1.1 illustrates this, showing that the projection of a family of FDs need not be characterized by FDs.

The main result on form which is needed in this paper states that the projection of a family of EGDs is always characterized by a family of EGDs [Fag82, Thm. 6.1]. In other words, if the main schema is constrained by EGDs, and the view is a projection, then the view schema has a basis for its constraints consisting entirely of EGDs.

6.3 Single relation EGD-schemata and their views and decompositions While the theory developed here applies to multi-relation schemata, such schemata are constructed by assembling a set of single-relation schemata, each with its own set of EGDs, and then imposing a common set of UINDs. Therefore, it is appropriate to begin with a careful defi nition of single-relation schemata.

(a) A single-relation EGD-schema is a pair $(R[\mathbf{U}], \Phi)$ in which $R$ is a relation name on attribute set $\mathbf{U}$ and $\Phi$ is a fi nite set of EGDs on $R[\mathbf{U}]$.

(b) Given a single relation EGD-schema $\mathbf{R}=(R[\mathbf{U}], \Phi)$ and $\mathbf{W} \subseteq \mathbf{U}$, the (projection) view defined by $\mathbf{W}$ is $\Pi_{\mathbf{W}}=\left(\left(R[\mathbf{W}], \pi_{\mathbf{W}}(\Phi)\right), \pi_{\mathbf{W}}\right) . \pi_{\mathbf{W}}(\Phi)$ is the projection of the constraints $\Phi$ onto $\mathbf{W}$, and $\pi_{\mathbf{W}}: R[\mathbf{U}] \rightarrow R[\mathbf{W}]$ is the projection mapping of $R$ from the attributes of $\mathbf{U}$ to the attributes of W.

Although it is often the case that the EGDs of the schema will ensure that the decomposition is lossless (that the EGDs defi ne a lossless decomposition was a requirement in the earlier work [Heg04b]), it is not necessary to so require. As expressed in general form in 4.6, the dependency which defi nes the reconstruction may be specifi ed separately. By its nature, this dependency is not embeddable in the views. In the single-relation case, it is always a join dependency; the following defi nition makes this explicit.

(c) A single-relation EGD-schema with $J D$ is a triple $\left(R[\mathbf{U}], \Phi, \bowtie\left[\mathbf{W}_{1}, \mathbf{W}_{2}\right]\right)$ in which $(R[\mathbf{U}], \Phi)$ is a single-relation EGD-schema and $\bowtie\left[\mathbf{W}_{1}, \mathbf{W}_{2}\right]$ is a full join dependency on $\mathbf{U}$; i.e., $\mathbf{W}_{1}, \mathbf{W}_{2} \subseteq \mathbf{U}$ with $\mathbf{W}_{1} \cup \mathbf{W}_{2}=\mathbf{U}$. The possibility that either of $\mathbf{W}_{1}$ and $\mathbf{W}_{2}$ is empty, or that $\mathbf{W}_{1} \cap \mathbf{W}_{2}=\emptyset$, is not excluded.

(d) The single-relation EGD-schema with JD $\left(R[\mathbf{U}], \Phi, \bowtie\left[\mathbf{W}_{1}, \mathbf{W}_{2}\right]\right)$ defines a meet-complementary pair if $\left\{\Pi_{\mathbf{W}_{1}}, \Pi_{\mathbf{W}_{2}}\right\}$ forms a meet-complementary pair of views of $(R[\mathbf{U}], \Phi)$.

Everything about EGD-schemata is decidable and constructible by algorithm, as shown by the next result.

6.4 Proposition - Algorithmic construction of covers for EGDs $\quad$ Let $\mathbf{R}=\left(R[\mathbf{U}], \Phi, \bowtie\left[\mathbf{W}_{1}, \mathbf{W}_{2}\right]\right)$ be a single-relation EGD schema with JD which defines a meet-complementary pair $\left\{\Pi_{\mathbf{W}_{1}}, \Pi_{\mathbf{W}_{2}}\right\}$, and let $k \in \mathbb{N}$.

(a) $\mathbf{R}$ has the $\left\{\Pi_{\mathbf{W}_{1}}, \Pi_{\mathbf{W}_{2}}\right\}$-relative $k$-submodel property iff $\Phi$ has a basis consisting of EGDs of degree at most $k$.

(b) It is decidable whether or not $\mathbf{R}$ has the $\left\{\Pi_{\mathbf{W}_{1}}, \Pi_{\mathbf{W}_{2}}\right\}$-relative $k$-submodel property. 
(c) If $\mathbf{R}$ has the $\left\{\Pi_{\mathbf{W}_{1}}, \Pi_{\mathbf{W}_{2}}\right\}$-relative $k$-submodel property, then it is possible to construct a set $\Psi$ of $E G D$ s of degree no more than $k$ with the property that $\Psi \cup\left\{\bowtie\left[\mathbf{W}_{1}, \mathbf{W}_{2}\right]\right\}$ and $\Phi \cup\left\{\bowtie\left[\mathbf{W}_{1}, \mathbf{W}_{2}\right]\right\}$ are equivalent sets of dependencies (for finite or infinite relations).

Proof: To establish (a), let $\Phi^{\prime}$ be the set of all EGDs on $\mathbf{R}$ which are of degree at most $k$, and which are consequences of those in $\Phi$. Note that $\Phi^{\prime}$ is a fi nite set, up to renaming of variables, since the number of distinct patterns of equality of variables on the left hand side is fi nite. Now, let $M \in \mathrm{DB}(\mathbf{D})$ satisfy all dependencies in $\Phi$. Then, $M$ is a $\left\langle\Gamma_{1} ; \Gamma_{2}\right\rangle$-relative $k$-submodel, by construction, and hence a model. Thus, $\Phi^{\prime}$ itself must be a basis for $\Phi$, as required.

For (b), let $\Phi^{\prime \prime}$ be the set of all EGDs on $\mathbf{R}$ which are of degree at most $k$. This is a fi nite set, for the same reason as $\Phi^{\prime}$ above is - there are only a fi nite number of possible patterns for the left hand side of such a constraint. Now, for each such constraint $\varphi \in \Phi^{\prime \prime}$, simply test to see whether $\Phi \models \varphi$, using any of the standard inference procedures for data dependencies, such as the chase of [BV84a] or the resolution-like procedure of [GJ82]. Since these are all universal dependencies, inference for infi nite and fi nite relations are equivalent. Part (c) follows immediately from this.

6.5 Multi-relation EGD-schemata and their views and decompositions In part (a) below, a multi-relation EGD schema is nothing more than a suitably presented collection of single-relation EGD schemata. In part (b), a common set of (inter-relational) UINDs is imposed on top of this to obtain the formalization of the schemata to be investigated in this section.

(a) A multi-relation EGD-schema with JDs is a set $\left\{\left(R_{i}\left[\mathbf{U}_{i}\right], \Phi_{i}, \bowtie\left[\mathbf{W}_{1 i}, \mathbf{W}_{2 i}\right]\right) \mid 1 \leq i \leq n\right\}$ in which $\left(R_{i}\left[\mathbf{U}_{i}\right], \Phi_{i}, \bowtie\left[\mathbf{W}_{1 i}, \mathbf{W}_{2 i}\right]\right)$ is a single-relation EGD-schema for $1 \leq i \leq n$, and the attributes are pairwise disjoint, in the precise sense that for $i \neq j, \mathbf{U}_{i} \cap \mathbf{U}_{j}=\emptyset$.

(b) A multi-relation EGD-schema with JDs and UINDs is a pair $\left(\left\{\left(R_{i}\left[\mathbf{U}_{i}\right], \Phi_{i}, \bowtie\left[\mathbf{W}_{1 i}, \mathbf{W}_{2 i}\right]\right) \mid 1 \leq\right.\right.$ $\left.i \leq n\}, \Phi_{\mathrm{UIND}}\right)$ in which $\left\{\left(R_{i}\left[\mathbf{U}_{i}\right], \Phi_{i}, \bowtie\left[\mathbf{W}_{1 i}, \mathbf{W}_{2 i}\right]\right) \mid 1 \leq i \leq n\right\}$ is a multi-relation EGD schema with JDs and $\Phi_{\text {UIND }}$ is a set of UINDs on these relations.

6.6 Axioms of inference for UINDs in the presence of FIDs As already remarked in 6.1, the interaction of FIDs and UINDs is minimal. The following inference rules, from [CKV90]; apply to single-relation schemata constrained by FIDs and UINDs. (Since both EGDs and JDs are FIDs, these rules apply to the context of this paper.) They are presented in the standard format in which the preconditions of the rule are presented above the horizontal line, with the consequences below it.

(a) Reflexivity and transitivity of UINDs:

(rt-uid)

$$
\frac{A \sqsubseteq B \quad B \sqsubseteq C}{A \sqsubseteq A} \quad \frac{A \sqsubseteq C}{A}
$$

(b) The cycle rule for FDs and UINDs on fi nite databases:

(cy-uid)

$$
\begin{array}{lllll}
C_{0} \rightarrow C_{1} & C_{1} \sqsupseteq C_{2} & \ldots & C_{m-1} \rightarrow C_{m} & C_{m} \sqsupseteq C_{0} \\
\hline C_{1} \rightarrow C_{0} & C_{2} \sqsupseteq C_{1} & \ldots & C_{m} \rightarrow C_{m-1} & C_{0} \sqsupseteq C_{m}
\end{array}
$$

The cycle rule is peculiar to the context of fi nite databases, and does not apply in the infi nite case. It is best understood in terms of a special case. Suppose that both $C_{0} \rightarrow C_{1}$ and $C_{1} \sqsubseteq C_{0}$ hold. For 
a relation $r$ which satisfi es these constraints, The FD forces $\operatorname{Card}\left(\pi_{C_{1}}\right)(r) \leq \operatorname{Card}\left(\pi_{C_{0}}(r)\right)$, while the UIND forces $\operatorname{Card}\left(\pi_{C_{0}}\right)(r) \leq \operatorname{Card}\left(\pi_{C_{1}}(r)\right)$. Thus, $\operatorname{Card}\left(\pi_{C_{0}}\right)(r)=\operatorname{Card}\left(\pi_{C_{1}}(r)\right)$, and combined with the inclusion implied by the UIND, this forces the two sets to be equal, thus implying $C_{1} \rightarrow C_{0}$ and $C_{0} \sqsubseteq C_{1}$. The rule (cy-uid) states this idea in a more general form in which the FDs and UINDs may chain over several relations. This is the only way in which FIDs may combine with UINDs to form new constraints of either class. A formalization of these ideas is given next.

6.7 Lemma - Decoupling of FIDs and UINDs Let $(R[\mathbf{U}], \Phi)$ be a single-relation schema in which $\Phi=\Phi_{\mathrm{FID}} \cup \Phi_{\mathrm{UIND}}$, with $\Phi_{\mathrm{FID}}$ a set of FIDs, and $\Phi_{\mathrm{UIND}}$ a set of UINDs, both taken over the attribute set $\mathbf{U}$. Let $\varphi$ be any FID or UIND over $R[\mathbf{U}]$.

(a) $\Phi \models_{f} \varphi$ holds iff $\Phi \vdash \varphi$ may be established using standard inference rules for FIDs (such as the chase [BV84a] or the resolution-like inference procedure of [GJ82]), together with the rules (rt-uid) and (cy-uid) given in 6.6.

(b) Using this proof procedure, it is decidable whether or not $\Phi \models_{f} \varphi$ holds.

Here, $\models_{f}$ denotes finite implication; that is, implication when the models are finite relations, and $\vdash$ denotes deduction via the above-mentioned proof procedures.

Proof: See [CKV90, Corollary 5.3]. Note in particular that this result applies to finite models, which is exactly the framework used here.

6.8 Product-relation EGD schemata and EJU/EGD-schemata The results identifi ed in 6.7 above apply in the context of single-relation schemata, while the focus of investigation here is multirelation schemata. The easiest remedy to this problem is to recast (temporarily) the framework of this section into a single-relation format. In this process, a minor additional constraint will be imposed.

The idea is simple - just form the Cartesian product of the component relations using the socalled cross dependency $\bowtie\left[\mathbf{U}_{1}, \ldots, \mathbf{U}_{n}\right]$, which is just a join dependency in which there are no common columns of the component attributes. More precisely, let $\mathbf{R}=\left(\left\{\left(R_{i}\left[\mathbf{U}_{i}\right], \Phi_{i}, \bowtie\left[\mathbf{W}_{1 i}, \mathbf{W}_{2 i}\right]\right) \mid 1 \leq i \leq\right.\right.$ $\left.n\}, \Phi_{\text {UIND }}\right)$ be a multi-relational EGD-schema with JDs and UINDs.

(a) The product-relation EGD schema with JDs and UINDs corresponding to $\mathbf{R}$ is

$$
\text { (mregd) }\left(R_{1}\left[\mathbf{U}_{1}\right] \times \ldots \times R_{n}\left[\mathbf{U}_{n}\right],\left(\bigcup_{i=1}^{n} \Phi_{i}\right) \cup \Phi_{\mathrm{UIND}} \cup\left\{\bowtie\left[\mathbf{W}_{1 i}, \mathbf{W}_{2 i}\right] \mid 1 \leq i \leq n\right\} \cup\left\{\bowtie\left[\mathbf{U}_{1}, \ldots, \mathbf{U}_{n}\right]\right\}\right)
$$

It is important to note in the above that the JDs in $\left\{\bowtie\left[\mathbf{W}_{1 i}, \mathbf{W}_{2 i}\right] \mid 1 \leq i \leq n\right\}$ may be viewed as full (as opposed to embedded) dependencies. This is true because the embedding is into a Cartesian product, and each JD lies in one component of this product. Indeed, defi ne $\overline{\mathbf{W}}_{i}=\bigcup_{i \neq j}\left(\mathbf{W}_{1 j} \cup \mathbf{W}_{2 j}\right)$, and then replace $\bowtie\left[\mathbf{W}_{1 i}, \mathbf{W}_{2 i}\right]$ with $\bowtie\left[\mathbf{W}_{1 i} \cup \overline{\mathbf{W}}_{i}, \mathbf{W}_{2 i} \cup \overline{\mathbf{W}}_{i}\right]$. In the presence of $\bowtie\left[\mathbf{U}_{1}, \ldots, \mathbf{U}_{n}\right]$, these two constraints are equivalent, with the latter being an FID. Thus, all of the constraints, save for those in $\Phi_{\text {UIND }}$, are FIDs.

There is a small complication; namely, this approach will not work if some of the relations are empty and others are not. One way around this, taken in [FV83], is to add a special tuple to each relation, and require that these special tuples always be present. Here, a simpler approach is taken, in which the requirement is imposed that if one relation is nonempty, then they all must be nonempty. In practice, this is hardly a serious drawback, since relations in real databases are almost never empty. 
(b) Defi ne the uniform nonemptiness-emptiness constraint $\varphi_{\mathrm{NE}}$ for $\mathbf{R}$ to be that which states that for all $i \in\{1,2, \ldots, n\}$, it is either the case the the instance of every $R_{i}$ is empty, or else the case the instance of every $R_{i}$ is nonempty.

(c) The uniform nonemptiness-emptiness schema corresponding to $\mathbf{R}$ is

$$
(\text { eju/egd }) \quad\left(\left\{\left(R_{i}\left[\mathbf{U}_{i}\right], \Phi_{i}, \bowtie\left[\mathbf{W}_{1 i}, \mathbf{W}_{2 i}\right]\right) \mid 1 \leq i \leq n\right\}, \Phi_{\mathrm{UIND}} \cup\left\{\varphi_{\mathrm{UNE}}\right\}\right)
$$

(d) A multi-relation EGD-schema with JDs and UINDs and with the uniform nonemptinessnonemptiness constraint is exactly a schema of the above form. For convenience, such a schema will also be called an EJU/EGD-schema.

6.9 Theorem - Constraint interaction on EJU/EGD-schemata Let $\mathbf{R}$ be an EJU/EGD-schema of the form given in (eju/egd) of 6.8. Then it is possible to construct, by algorithm, finite sets of constraints $\left\{\Phi_{1}^{\prime}, \Phi_{2}^{\prime}, \ldots, \Phi_{n}^{\prime}, \Phi_{U I N D}^{\prime}\right\}$ with the following properties.

(a) With $\mathbf{R}^{\prime}$ defined as

$$
\left(\left\{\left(R_{i}\left[\mathbf{U}_{i}\right], \Phi_{i}^{\prime}, \bowtie\left[\mathbf{W}_{1 i}, \mathbf{W}_{2 i}\right]\right) \mid 1 \leq i \leq n\right\}, \Phi_{U I N D}^{\prime} \cup\left\{\varphi_{U N E}\right\}\right)
$$

it is the case that $\operatorname{LDB}(\mathbf{R})=\operatorname{LDB}\left(\mathbf{R}^{\prime}\right)$.

(b) For each $i \in\{1,2, \ldots, n\}$, if $\varphi$ is an EGD on $\mathbf{R}_{i}$ with $\left(\left(\bigcup_{i=1}^{n} \Phi\right) \cup \Phi_{U I N D}\right) \models_{f} \varphi$, then $\Phi_{i}^{\prime} \models_{f} \varphi$.

(b) If $\varphi$ is a UIND on $\bigcup_{i=1}^{n} \mathbf{U}_{i}$ and $\left(\left(\bigcup_{i=1}^{n} \Phi\right) \cup \Phi_{U I N D}\right) \models_{f} \varphi$, then $\varphi \in \Phi_{U I N D}^{\prime}$.

PROOF: Follows directly from 6.7 and the equivalence between product- and multiple-relational schemata developed in 6.8. The only point of concern is the effect of the join dependencies. However, according to [BV84b, Lem. 7], the join dependencies in $\left\{\bowtie\left[\mathbf{W}_{1 i} \cup \overline{\mathbf{W}}_{i}, \mathbf{W}_{2 i} \cup \overline{\mathbf{W}}_{i}\right] \mid 1 \leq i \leq\right.$ $n\} \cup\left\{\bowtie\left[\mathbf{U}_{1}, \ldots, \mathbf{U}_{n}\right]\right\}$ cannot cause any additional EGDs to be generated that would not already be generated by the EGDs alone. Since UINDs and EGDs only interact via FDs, it follows that the JDs are inconsequential in terms of constructing the $\Phi_{i}^{\prime}$ s and $\Phi_{\text {UIND }}^{\prime}$.

6.10 Notational and constraint-format convention For the rest of this section, unless stated specifi cally to the contrary, $\mathbf{R}$ will be taken to be an EJU/EGD-schema of the form shown in (eju/egd) of 6.8. For the sake of convenience, it will also be assumed that the $\Phi_{i}$ 's and $\Phi_{\text {UIND }}$ satisfy the conditions identifi ed in 6.9 above. In particular, $\Phi_{\text {UIND }}$ will contain all UINDs which govern the schema.

Also, since the context $\Phi_{\text {UIND }}$ is fi xed, for $A_{1}, A_{2} \in \mathbf{U}$, the statement $A_{1} \sqsubseteq A_{2}$ will be used as an abbreviation for the more cumbersome $A_{1} \sqsubseteq A_{2} \in \Phi_{\text {UIND }}$.

6.11 Notation for the views and decomposition of an EJU/EGD schema In the general context of the previous sections, the decomposition of the main schema $\mathbf{D}$ was denoted $\left\{\Gamma_{1}, \Gamma_{2}\right\}$, with the meet of this meet-complementary pair denoted $\Gamma_{3}$. Similarly, a standard notation will be used for the relational case considered here. The component views of the EJU/EGD schema $\mathbf{R}$ will be denoted $\left\{\Gamma_{\pi_{1}(\mathbf{R})}, \Gamma_{\pi_{2}(\mathbf{R})}\right\}$. These two will be taken to be a meet-complementary pair, with meet $\Gamma_{\pi_{3}(\mathbf{R})}$. More formally, proceed as follows.

(a) For $i \in\{1,2\}$, defi ne the schema $\pi_{i}(\mathbf{R})=\left\{\left(R_{i}\left[\mathbf{W}_{i j}\right], \pi_{\mathbf{W}_{i j}}\left(\bigcup_{m=1}^{n}\left(\Phi_{i m}\right) \cup \Phi_{\mathrm{UIND}}\right)\right) \mid 1 \leq j \leq n\right\}$. 
(b) Defi ne the schema $\pi_{3}(\mathbf{R})=\left\{\left(R_{i}\left[\mathbf{W}_{1 i} \cap \mathbf{W}_{2 i}\right], \pi_{\mathbf{W}_{1 i} \cap \mathbf{W}_{2 i}}\left(\bigcup_{m=1}^{n}\left(\Phi_{i m}\right) \cup \Phi_{\text {UIND }}\right)\right) \mid 1 \leq j \leq n\right\}$.

At this point, the sets of the form $\pi_{\mathbf{Z}}\left(\bigcup_{m=1}^{n}\left(\Phi_{i m}\right) \cup \Phi_{\text {UIND }}\right)$ are simply some representation of the constraints which project from $\mathbf{R}$. It remains to characterize their exact nature.

(c) For $i \in\{1,2\}$, the mapping $\pi_{i}^{\mathbf{R}}: \mathbf{R} \rightarrow \pi_{i}(\mathbf{R})$ is just the collection of the projections $\left\{\pi_{\mathbf{W}_{i}}\right.$ : $\left.R\left[\mathbf{U}_{j}\right] \rightarrow R\left[\mathbf{W}_{i j}\right] \mid 1 \leq j \leq n\right\}$. For $i=3, \pi_{3}^{\mathbf{R}}: \mathbf{R} \rightarrow \pi_{3}(\mathbf{R})$ is $\left\{\pi_{\mathbf{W}_{3}}: R\left[\mathbf{U}_{j}\right] \rightarrow R\left[\mathbf{W}_{1 j} \cap \mathbf{W}_{2 j}\right] \mid 1 \leq\right.$ $j \leq n\}$.

(d) For $i \in\{1,2,3\}$, the view $\Gamma_{\pi_{i}(\mathbf{R})}=\left(\pi_{i}(\mathbf{R}), \pi_{i}^{\mathbf{R}}\right)$.

(e) For $i \in\{1,2\}$, defi ne $\mathbf{U}^{\dot{ }}=\bigcup_{j=1}^{n} \mathbf{W}_{i j}$, and defi ne $\mathbf{U}^{\beta}=\mathbf{U}^{1} \cap \mathbf{U}^{2}$. Furthermore, for $i \in\{1,2,3\}$, let $\mathbf{U}^{\bar{i}}=\mathbf{U} \backslash \mathbf{U}^{i}$,

6.12 Further notational convention Unless stated specifi cally to the contrary, throughout the remainder of this section, $\left\{\Gamma_{\pi_{1}(\mathbf{R})}, \Gamma_{\pi_{2}(\mathbf{R})}\right\}$ will be assumed to be a meet-complementary pair, with meet $\Gamma_{\pi_{3}(\mathbf{R})}$.

6.13 The graphs of $\Phi_{\text {UIND }}$ The family $\Phi_{\text {UIND }}$ defi nes a directed graph in a natural way, with the vertices the attributes in $\mathbf{U}$. An edge from $A_{1}$ to $A_{2}$ in this graph corresponds to $A_{1} \sqsubseteq A_{2}$, provided that this dependency cannot be inferred by transitive closure. In other words, the graph defi nes a minimal "skeleton" of the UINDs, from which the others may be derived via the transitivity rule of (rt-uid) of 6.6.

(a) Formally, the graph of $\Phi_{U I N D}$, denoted $\operatorname{Graph}\left(\Phi_{\text {UIND }}\right)$, is defi ned as follows.

(i) The set of vertices of $\operatorname{Graph}\left(\Phi_{\mathrm{UIND}}\right)$ is just $\mathbf{U}$, the set of all attributes over the relations.

(ii) For $A_{1}, A_{2} \in \mathbf{U}$, there is a (directed) edge from $A_{1}$ to $A_{2}$ precisely in the case that $A_{1} \sqsubseteq A_{2}$ and, for all $A_{3} \in \mathbf{U}$ with the property that $A_{1} \sqsubseteq A_{3} \sqsubseteq A_{2}$, either $A_{1}=A_{2}$ or else $A_{2}=A_{3}$. Write $A_{1} \prec A_{2}$ to indicate that there is an edge from $A_{1}$ to $A_{2}$ in $\operatorname{Graph}\left(\Phi_{\mathrm{UIND}}\right)$.

It is important to be able to classify attributes (qua vertices) according to the projections in which they lie. The following provides a convenient notation for this.

(b) For $i \in\{1,2,3, \overline{1}, \overline{2}, \overline{3}\}$ and $A \in \mathbf{U}, A$ is an $(i)$-vertex precisely in the case that $A \in \mathbf{U}^{i}$.

The graph $\operatorname{Graph}\left(\Phi_{\mathrm{UIND}}\right)$ may contain cycles, and it is important to have a means of grouping the corresponding vertices together, since they will all have exactly the same projection in any legal database. The reduced graph of $\Phi_{\mathrm{UIND}}$ collapses $\operatorname{Graph}\left(\Phi_{\mathrm{UIND}}\right)$ by collecting such equivalent vertices into one.

(c) The vertices $A_{1}, A_{2} \in \mathbf{U}$ are equivalent if both $A_{1} \sqsubseteq A_{2}$ and $A_{2} \sqsubseteq A_{1}$ hold. The equivalence class of $A_{1}$ under this relation is denoted $\left[A_{1}\right]$.

(d) The reduced graph of $\Phi_{U I N D}$, denoted [Graph] $\left(\Phi_{\mathrm{UIND}}\right)$, is defi ned as follows.

(i) The set of vertices of $\operatorname{Graph}\left(\Phi_{\text {UIND }}\right)$ is just $\{[A] \mid A \in \mathbf{U}\}$. Denote this set by $[\mathbf{U}]$.

(ii) For $\left[A_{1}\right],\left[A_{2}\right] \in[\mathbf{U}]$, there is a (directed) edge from $\left[A_{1}\right]$ to $\left[A_{2}\right]$ precisely in the case that $\left[A_{1}\right] \neq\left[A_{2}\right]$ and $B_{1} \prec B_{2}$ in $\operatorname{Graph}\left(\Phi_{\mathrm{UIND}}\right)$ for some $B_{1} \in\left[A_{1}\right]$ and $B_{2} \in\left[A_{2}\right]$. Write $\left[A_{1}\right] \prec\left[A_{2}\right]$ to indicate that there is an edge from $\left[A_{1}\right]$ to $\left[A_{2}\right]$ in $[\mathrm{Graph}]\left(\Phi_{\mathrm{UIND}}\right)$. 
The identifi cation process of (b) above extends to the reduced graph, but two possibilities must be considered. The fi rst occurs when some element from the equivalence class lies in a given region, and the other when all elements from that equivalence class do.

(e) For $i \in\{1,2,3, \overline{1}, \overline{2}, \overline{3}\}$ and $[A] \in[\mathbf{U}], A$ is an $(\exists i)$-vertex in the case that some $B \in[A]$ is an $(i)$-vertex in $\operatorname{Graph}\left(\Phi_{\mathrm{UIND}}\right)$, and a $(\forall i)$-vertex in the case that every $B \in[A]$ is an $(i)$-vertex in $\operatorname{Graph}\left(\Phi_{\mathrm{UIND}}\right)$.

6.14 Fanout-free families of UINDs To realize the main result of this section, it is necessary to place a restriction on the form of allowed family of UINDs. The requirement stipulates that there cannot be branching in the forward direction. Formally, the condition is formulated as follows.

(a) The set $\Phi_{\text {UIND }}$ is fanout free if for any $A_{1}, A_{2}, A_{3} \in \mathbf{U}$, if both $A_{1} \sqsubseteq A_{2}$ and $A_{1} \sqsubseteq A_{3}$ hold, then one of $A_{2} \sqsubseteq A_{3}$ or $A_{3} \sqsubseteq A_{2}$ holds as well.

It does not appear that restricting consideration to fanout free systems of UINDs is a signifi cant limitation in practice. Consider in particular the ubiquitous foreign-key constraints or referential integrity constraints [EN04, Sec. 5.2.4]. The inclusion $A_{2} \sqsubseteq A_{1}$ may be used to model the situation in which $A_{2}$ of ( $R_{2}$, say) is a foreign key to the primary key $A_{1}$ (of $R_{1}$, say). There may certainly be another relation $\left(R_{3}\right.$, say) with a foreign key $A_{3}$ which also references the primary key $A_{1}$ of $R_{1}$. Fanout freeness does not prohibit this. What it would prohibit would be a situation in which a foreign key had to be a subset of two distinct primary keys, but this is not part of traditional database modelling.

Of course, primary keys consisting of more than one attribute are allowed in existing database systems, although they are relatively uncommon. These are not modellable directly within the framework developed here, although in real instances of such, the multiple attributes could likely be lumped into one, for the purposes of modelling the dependencies of the schema.

6.15 Meet-free traversals and meet-situated ancestors and descendants The question of which UINDs may be allowed and which must be prohibited in a meet-complementary decomposition is now considered. The only type of UIND which is does not obviously embed into one of the two component views of the decomposition is one which connects an attribute in $\mathbf{U}^{\overline{2}}$ to one in $\mathbf{U}^{\overline{1}}$; that is, one whose two attributes lie in opposite views, with neither lying in the meet. (More precisely, these two attributes cannot be equivalent to any which lie in the meet.) The formalization of this situation is as follows.

(a) The edge $A_{1} \prec A_{2}$ of $\operatorname{Graph}\left(\Phi_{\text {UIND }}\right)$ defi nes a meet-free traversal from $\Gamma_{\pi_{1}(\mathbf{R})}$ to $\Gamma_{\pi_{2}(\mathbf{R})}$ if one of the following two conditions is satisfi ed.

(i) $\left[A_{1}\right]$ is a type $(\forall \overline{2})$-vertex and $\left[A_{2}\right]$ is a type $(\forall \overline{1})$-vertex of $[\mathrm{Graph}]\left(\Phi_{\text {UIND }}\right)$.

(ii) $\left[A_{1}\right]=\left[A_{2}\right], A_{1}$ is a $(\overline{2})$-vertex, $A_{2}$ is a $(\overline{1})$ vertex, and for all $B \in\left[A_{1}\right], B$ is either a $(\overline{1})$-vertex or else a $(\overline{2})$-vertex of $[\mathrm{Graph}]\left(\Phi_{\mathrm{UIND}}\right)$.

(b) A meet-free traversal from $\Gamma_{\pi_{2}(\mathbf{R})}$ to $\Gamma_{\pi_{1}(\mathbf{R})}$ is defi ned analogously.

The goal is to show that meet-free traversals cannot occur in the case that $\left\{\Gamma_{\pi_{1}(\mathbf{R})}, \Gamma_{\pi_{2}(\mathbf{R})}\right\}$ is a meetcomplementary pair. The idea is simple. If $A_{1} \prec A_{2}$ defi nes a meet-free traversal from $\Gamma_{\pi_{1}(\mathbf{R})}$ to $\Gamma_{\pi_{2}(\mathbf{R})}$, 
and $M \in \operatorname{LDB}(\mathbf{R})$, then there is an $a \in \pi_{A_{1}}(M)$ which must necessarily be in $\pi_{A_{2}}(M)$ as well. Now, replace this $a$ with some $b \in \operatorname{Dom}(\mathbf{U})$ which does not occur in $M$ to obtain a new model $M^{\prime}$. Then, consider $M^{\prime \prime}=\left(\pi_{1}^{\mathbf{R}} \otimes \pi_{2}^{\mathbf{R}}\right)^{-1}\left(\pi_{1}^{\mathbf{R}}(M), \pi_{2}^{\mathbf{R}}\left(M^{\prime}\right)\right)$ which must also be in $\operatorname{LDB}(\mathbf{R})$, since it is constructed from the appropriate view projections of $M$ and $M^{\prime}$. It is clear that $M^{\prime \prime}$ cannot satisfy $A_{1} \sqsubseteq A_{2}$, whence a contradiction is obtained. Thus, such a meet-free traversal cannot occur, and so there must be a basis of the UINDs which embed in the views of the decomposition.

Unfortunately, there is a flaw in the above argument. Namely, when $a$ is replaced by $b$, this may change the state of the meet view $\Gamma_{\pi_{3}(\mathbf{R})}$, since $a$ may have been visible there as well, due to the presence of other UINDs. Such a change would render the pair $\left(\pi_{1}^{\mathbf{R}}(M), \pi_{2}^{\mathbf{R}}\left(M^{\prime}\right)\right)$ join incompatible, and prevent the construction of $M^{\prime \prime}$. Fortunately, there is a way to ensure that this does not happen. The details are now presented.

Informally, a minimal proper meet-situated ancestor of $A_{1}$ is a predecessor of $A_{2}$ (i.e., $A_{2} \sqsubseteq A_{1}$ which lies in the meet $\Gamma_{\pi_{3}(\mathbf{R})}$, with the additional constraint that no attribute "between" between $A_{2}$ and $A_{1}$ has this property. A minimal proper meet-situated successor is defi ned dually; i.e., in the opposite direction of inclusion. It should be noted that a minimal proper meet-situated successor must be unique, if it exists, due to the fanout-freeness condition.

In the defi nitions below, let $i \in\{1,2\}$, and let $A_{1} \in \mathbf{U}^{\bar{i}}$.

(c) $A_{2}$ is a minimal proper meet-situated ancestor of $A_{1}$ if the following conditions are met.

(i) $A_{2} \sqsubseteq A_{1}$.

(ii) $A_{2}$ is a (3)-vertex; (i.e., $A_{2}$ lies in the meet $\Gamma_{\pi_{3}(\mathbf{R})}$ ).

(iii) Whenever $A_{2} \sqsubseteq A_{3} \sqsubseteq A_{1}$ holds with $\left[A_{1}\right] \neq\left[A_{3}\right] \neq\left[A_{2}\right]$, it must be the case that $\left[A_{3}\right]$ is a $(\forall \overline{3})$-vertex; (i.e., $A_{2}$ does not lie in the meet $\left.\Gamma_{\pi_{3}(\mathbf{R})}\right)$.

(d) $A_{2}$ is a minimal proper meet-situated successor of $A_{1}$ if conditions identical to (i)-(iii) above,

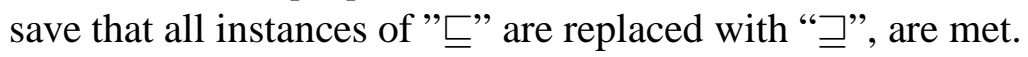

Now, the first of three lemmata which lead to the construction of the contradictory database $M^{\prime \prime}$ of 6.15 is presented.

6.16 Lemma Assume that $\Phi_{U I N D}$ is fanout free, let $i \in\{1,2\}$, and let $A_{1}, A_{2} \in \mathbf{U}$ with $A_{1} \prec A_{2} a$ meet-free traversal from $\pi_{i}(\mathbf{R})$ to $\pi_{2-i}(\mathbf{R})$. Let $M \in \mathrm{DB}(\mathbf{D})$, and let $a \in \operatorname{Dom}(\mathbf{U})$ be such that the following conditions are satisfied on $M$.

(i) $a \in \pi_{A_{1}}(M)$.

(ii) For no minimal proper meet-situated ancestor $B$ of $A_{2}$ is it the case that $a \in \pi_{B}(M)$.

(iii) For any minimal proper meet-situated successor $B$ of $A_{2}$, there exists $b \in \pi_{B}(M)$ with $b \notin$ $\pi_{A_{2}}(M)$.

Then it cannot be the case that $M \in \operatorname{LDB}(\mathbf{R})$.

Proof: To begin, assume that $M \in \operatorname{LDB}(\mathbf{R})$; it will be shown that this assumption is contradictory. The idea is to replace $a$ by $b$ in certain place in $M$ to obtain a new $M^{\prime} \in \operatorname{LDB}(\mathbf{R})$ with the properties that $\pi_{\mathbf{U}_{3}}(M)=\pi_{\mathbf{U}_{3}}\left(M^{\prime}\right)$ and $M^{\prime} \in \operatorname{LDB}(\mathbf{R})$ iff $M \in \operatorname{LDB}(\mathbf{R})$. In particular, it will be the case that $\pi_{\mathbf{U}_{3}}(M)=\pi_{\mathbf{U}_{3}}\left(M^{\prime}\right)$. 
First of all, since it is assumed that $M \in \operatorname{LDB}(\mathbf{R})$, it must be the case that $a \in \pi_{A_{2}}(M)$, since the UIND $A_{1} \sqsubseteq A_{2}$ holds. Now assume that $A_{2}$ has a minimal proper meet-situated successor $B$. In view of the fanout-freeness assumption, all such ancestors must lie in the same equivalence class $[B]$. Choose $b \in \pi_{B}(M) \backslash \pi_{A_{2}}(M)$; such an element is guaranteed by (iii) above. Let $\left[A_{2}\right]=\left[B_{1}\right] \prec\left[B_{2}\right] \prec$ $\ldots \prec\left[B_{n}\right]=B$ be the path in $[\mathrm{Graph}]\left(\Phi_{\mathrm{UIND}}\right)$ from $\left[A_{2}\right]$ to $[B]$, and let $\left[B_{i}\right]$ be the last element in this path with $b \notin \operatorname{Dom}\left(B_{i}\right)$. Now, to obtain $M^{\prime}$ from $M$, for all attributes $C \in \mathbf{U}$ with $C \sqsubseteq B_{i}$, replace all occurrences of $a$ with $b$ in the $C$ position of all tuples containing that attribute with value $a$. (Note that this replacement procedure includes $B_{i}$ in particular.)

If $A_{2}$ does not have a minimal proper meet-situated successor, then simply choose $b$ to be any any domain element which does not already occur in $M$, and replace all occurrences of $a$ by $b$ in $M$ to obtain $M^{\prime}$.

Note four things. First of all, for tuple $t$ and $A \in \mathbf{U}$ in which $t[A]$ is changed from $a$ to $b$, the value $a$ did not occur as the $A$-value of any other tuple $t^{\prime}$ of $M$. Thus, this replacement cannot force any of the EGDs or the JDs to become unsatisfi ed, since these constraints are all typed. Within attribute $A$, this replacement amounts to a simple renaming. Second, all of the UINDs remain satisfi ed, just by construction. Third, by the assumption of (ii) above, none of these changes is visible in the meet $\pi_{\mathbf{U}_{3}}(\mathbf{R})$. Finally, note that this construction works even in the case that $\left[A_{1}\right]=\left[A_{2}\right]$. In this case, the minimal proper meet-situated predecessors and successors will be common to the two attributes, but this will not change the construction.

Thus, $M^{\prime} \in \operatorname{LDB}(\mathbf{R})$. Since $\pi_{3}^{\mathbf{R}}(M)=\pi_{3}^{\mathbf{R}}\left(M^{\prime}\right), M^{\prime \prime}=\left(\pi_{1}^{\mathbf{R}} \otimes \pi_{2}^{\mathbf{R}}\right)^{-1}\left(\pi_{1}^{\mathbf{R}}(M), \pi_{2}^{\mathbf{R}}\left(M^{\prime}\right)\right)$ is well defi ned, and must be in $\operatorname{LDB}(\mathbf{R})$ as well. However, it is clear that this latter element violates $A_{1} \sqsubseteq A_{2}$, since $a \in \pi_{A_{1}}\left(M^{\prime \prime}\right) \backslash \pi_{A_{2}}\left(M^{\prime \prime}\right)$. This is a contradiction, and so $M \notin \operatorname{LDB}(\mathbf{R})$, as was to be shown.

6.17 Products of databases Let $\left\{M_{i} \mid 1 \leq j \leq m\right\} \subseteq \mathrm{DB}(\mathbf{D})$. The product of $\prod_{i=1}^{m} M_{i}$ is obtained by taking the products of the relations componentwise. More specifically, for the relation symbol $R_{i}$ of $\mathbf{R}$, if $t_{i}=\left(a_{i 1}, a_{i 2}, \ldots, a_{i n_{i}}\right)$ is a tuple in the relation for $R_{i}$ in $M_{i}, 1 \leq i \leq m$, then $t=\left(\left\langle a_{11}, a_{21}, \ldots, a_{n_{i} 1}\right\rangle,\left\langle a_{12}, a_{22}, \ldots, a_{n_{i} 2}\right\rangle, \ldots,\left\langle a_{1 m}, a_{2 m}, \ldots, a_{n_{i} m}\right\rangle\right)$ is a tuple in the relation corresponding to $R_{i}$ in $\prod_{i=1}^{m} M_{i}$, and conversely. Note that this requires a renaming of the elements of the domain $\operatorname{Dom}(\mathbf{U})$, but this is not a signifi cant issue, since it is the form of the models, and not the names of the domain elements, which are of importance.

In [Fag82], the issues surrounding preservation of models on such products and the corresponding projection operations are studied extensively, and a strong correspondence between such preservation and Armstrong models is established. For the purposes of this paper, only the two simple results stated in the lemma below are needed.

\subsection{Lemma}

(a) If $\left\{M_{i} \mid 1 \leq i \leq n\right\}$ is a finite subset of $\operatorname{LDB}(\mathbf{D})$, then the product $\prod_{i=1}^{n} M_{i} \in \operatorname{LDB}(\mathbf{D})$ as well.

(b) Let $A_{1}, A_{2} \in \mathbf{U}$ with the property that $A_{1} \sqsubseteq A_{2} \notin \Phi_{\text {UIND. }}$. Then there is an $M \in \operatorname{LDB}(\mathbf{D})$ on which $A_{1} \sqsubseteq A_{2}$ does not hold.

PROOF: First of all, part (a) follows almost immediately from [Fag82, Thm. 7.2], in which it is shown that every schema constrained by constraints which are called extended embedded implicational dependencies (XEIDs) is closed under products. All of the constraints used here - EGDs, JDs, and UINDs - are special cases of XEIDs. The only caveat is that his result, as stated, holds only 
on databases for which none of the component relations is empty. However, it is easy to see that it also holds if the database in which all relations are empty is allowed. It is rather the case in which some relations are empty and others are not which causes problems, and that case has already been eliminated from the framework used here via the uniform nonemptiness-emptiness constraint $\varphi_{\mathrm{UNE}}$.

For part (b), it suffi ces to note that since $\left(\left(_{i=1}^{n}\left(\Phi_{i} \cup \bowtie\left[\mathbf{W}_{1 i}, \mathbf{W}_{2 i}\right]\right)\right) \cup \Phi_{\mathrm{UIND}}\right) \not \models_{f} A_{1} \sqsubseteq A_{2}$, there must be some $M \in \mathrm{DB}(\mathbf{D})$ which satisfi es all of the constraints in $\bigcup_{i=1}^{n}\left(\Phi_{i} \cup \bowtie\left[\mathbf{W}_{1 i}, \mathbf{W}_{2 i}\right]\right)$ but does not satisfy $A_{1} \sqsubseteq A_{2}$. This is exactly the $M$ which is needed for part (b).

6.19 Lemma For any $A \in \mathbf{U}$ there is an $M \in \operatorname{LBB}(\mathbf{R})$ and an $a \in \pi_{A}(M)$ with the following properties.

(i) For no $B \in \mathbf{U}$ with $[B] \prec[A]$ is it the case that $a \in \pi_{B}(M)$.

(ii) For no $B \in \mathbf{U}$ with $[A] \prec[B]$ is it the case that $\pi_{B}(M)=\pi_{A}(M)$.

Proof: Let $B \in \mathbf{U}$ with $[B] \prec[A]$; thus, in particular, $B \sqsubseteq A \in \Phi_{\text {UIND }}$ while $A \sqsubseteq B \notin \Phi_{\text {UIND. In view }}$ of 6.18(b), there is an $M_{B} \in \operatorname{LDB}(\mathbf{R})$ with the property that $A \sqsubseteq B$ is not satisfi ed by $M_{B}$. Thus, there is an $a_{B} \in \mathbf{U}$ with $a_{B} \in \pi_{A}\left(M_{B}\right) \backslash \pi_{B}\left(M_{B}\right)$. Similarly, for $B \in \mathbf{U}$ with $[A] \prec[B] ; A \sqsubseteq B \in \Phi_{\text {UIND }}$ while $B \sqsubseteq A \notin \Phi_{\mathrm{UIND}}$, and so there is an $M_{B} \in \operatorname{LDB}(\mathbf{R})$ with the property that $B \sqsubseteq A$ is not satisfi ed by $M_{B}$. Now, let $S^{\prime}=\{[B] \mid[B] \prec[A]\} \cup\{[B] \mid[A] \prec[B]\}$ and let $S$ be any subset of $S^{\prime}$ which selects at least one representative from each equivalence class. Defi ne $M=\prod_{B \in S} M_{B}$; by 6.18(a), $M \in \operatorname{LDB}(\mathbf{D})$. Let $a \in M$ be the element whose $B^{\text {th }}$ component is $a_{b}$. Then $a \notin \pi_{B}(M)$ for any $B$ with $[B] \prec[A]$, since $a_{B} \notin M_{B}$. Hence, $M$ satisfi es condition (i) above. Condition (ii) is in fact satisfi ed by every $M_{B}$, and so by the product in particular.

It is now possible to establish that meet-free traversals cannot exist when the UINDs are fanout free. This in turn leads to the conclusion that a cover of the UINDs embeds in the component views.

6.20 Proposition If $\Phi_{U I N D}$ is fanout free, then $\operatorname{Graph}\left(\Phi_{U I N D}\right)$ cannot contain any meet-free traversals.

Proof: $\quad$ Let $i \in\{1,2\}$, and let $A_{1}, A_{2} \in \mathbf{U}$ with $A_{1} \prec A_{2}$ a meet-free traversal from $\pi_{i}^{\mathbf{R}}$ to $\pi_{2-i}^{\mathbf{R}}$. Let $A=A_{1}$ in 6.19 , and obtain a database $M \in \operatorname{LDB}(\mathbf{R})$ satisfying the conditions (i) and (ii) of that lemma. Then $M$ also satisfi es the three conditions (i)-(iii) of 6.16 , and so cannot be in $\operatorname{LB}(\mathbf{R})$. This is a contradiction; hence, such a meet-free traversal cannot exist.

6.21 Embedded UINDs For $i \in\{1,2\}$, let $\pi_{i}^{\mathbf{R}}\left(\Phi_{\mathrm{UIND}}\right)$ denote the set of all UINDs which hold on $\pi_{i}^{\mathbf{R}}$

6.22 Proposition If $\Phi_{U I N D}$ is fanout free, then $\Phi_{U I N D}=\left(\pi_{i}^{\mathbf{R}}\left(\Phi_{U I N D}\right) \cup \pi_{2}^{\mathbf{R}}\left(\Phi_{U I N D}\right)\right)^{+}$. In other words, there is an embedded cover of $\Phi_{U I N D}$ into the two views of the meet complementary pair.

PROOF: It is clear that the only UINDs which do not embed into one of the two component views, or which cannot be deduced from such embedded UINDs via application of the transitivity rule of (rt-uid) 6.6, are those which correspond to meet-free traversals. However, by 6.20, such traversals cannot occur in the case that $\Phi_{\text {UIND }}$ is fanout free. 
Finally, the main decomposition result may be established. Just as the inference rules for FIDs and UINDs may be decoupled, so too does the constraint checking on the views of the decomposition decouple into two independent checks, one for the EGDs and the other for the UINDs.

6.23 Theorem - Decomposition in the presence of EGDs and fanout-free UINDs Assume that $\Phi_{U I N D}$ is fanout free, let $k \in \mathbb{N}$, and suppose that for each $j, 1 \leq j \leq n, \Phi_{i}$ consists of EGDs of degree at most $k$. Let $M \in \operatorname{JDB}_{\left\langle\Gamma_{1} ; \Gamma_{2}\right\rangle}(\overline{\mathbf{D}})$. Then $M \in \operatorname{LDB}(\mathbf{R})$ iff for both $i=1$ and $i=2, \pi_{i}^{\mathbf{R}}(M)$ satisfies all EGDs in $\pi_{i}^{\mathbf{R}}\left(\Phi_{i}\right)$ of degree at most $k$, as as well as all UINDs in $\pi_{i}^{\mathbf{R}}\left(\Phi_{U I N D}\right)$.

ProOF: First of all, consider the same candidate model $M \in \operatorname{JDB}_{\left\langle\Gamma_{1} ; \Gamma_{2}\right\rangle}(\overline{\mathbf{D}})$, but as a database in a modifi ed schema in which the UINDs in $\Phi_{\text {UIND }}$ are ignored. The resulting schema clearly has the $\left\{\Gamma_{\pi_{1}(\mathbf{R})}, \Gamma_{\pi_{2}(\mathbf{R})}\right\}$-relative $k$-submodel property; thus, in view of 5.19, the main theorem on decomposition of schemata with the $k$-submodel property, to verify that the EGDs in $\bigcup_{i=1}^{n} \Phi_{i}$ are satisfi ed, it suffi ces to check that for both $i=1$ and $i=2, \pi_{i}^{\mathbf{R}}(M)$ satisfi es all EGDs in $\pi_{i}^{\mathbf{R}}\left(\Phi_{i}\right)$ of degree at most $k$.

Now, restore the requirement of checking satisfaction of the UINDs. By 6.22, a cover of the UINDs must embed into the views. Thus, checking the satisfaction of the members of this cover suffi ces to verify that the constraints of $\Phi_{\mathrm{IIND}}$ are satisfi ed. Hence, $M \in \operatorname{LDB}(\mathbf{D})$.

Upon restricting the above theorem to the familiar territory of FDs, the following result is obtained. In essence, to check the correctness of a decomposed database, it suffi ces to verify that each of the component views satisfi es all embedded FDs and UINDs.

6.24 Corollary - Decomposition in the presence of FDs and fanout-free UINDs Assume that $\Phi_{\text {UIND }}$ is fanout free, let $k \in \mathbb{N}$, and suppose that for each $j, 1 \leq j \leq n, \Phi_{i}$ consists of FDs. Let $M \in \mathrm{JDB}_{\left\langle\Gamma_{1} ; \Gamma_{2}\right\rangle}(\overline{\mathbf{D}})$. Then $M \in \operatorname{LDB}(\mathbf{R})$ iff for both $i=1$ and $i=2, \pi_{i}^{\mathbf{R}}(M)$ satisfies all FDs and UINDs from $\left(\Phi_{i}\right)^{+} \cup \Phi_{\text {UIND }}$ which embed in the view $\pi_{i}(\mathbf{R})$.

\section{Final Remarks}

7.1 Future Direction - An alternative approach to recapturing generating constraints The decomposition results for schemata constrained by comparison constraints (5.19) are general and comprehensive. On the other hand, the general results reported for generating constraints (5.25) are not as broad. While the results established in Section 6 on schemata constrained by EGDs and fanout-free UINDs nonetheless provide interesting results for what is perhaps the most important type of generating constraint in databases, they make use of very specifi c properties of the relational data model, and in particular of constraints governed by situations in which fi nite Armstrong relations exist. A crucial next step in this work is to look for alternative formulations of the general problem which promise to lead to stronger results about decompositions in the presence of generating constraints.

7.2 Future Direction - Strong uniqueness of complements In the preliminary version of this paper [Heg04b], results on the uniqueness of update strategies were also presented. One of the weaknesses of the classical constant-complement strategy, set in the set-based framework, is that complements are almost never unique, and the way in which view updates are reflected back to the main 
schema depends upon the choice of complement. The principal focus of [Heg04a] was to identify situations in which such uniqueness may be obtained. The setting was database schemata with order, and the main result stated that so-called order-based updates, that is, updates which may be realized as a sequence of legal insertions and deletions, have a unique reflection to the main view, independent of the choice of complement [Heg04a, 4.3].

In [Heg04b, Thm. 5.8], it was shown how to generalize this result in the context of CFA-views. The key idea was to extend update strategies on a view $\Gamma=(\mathbf{V}, \gamma)$ to its syntactic extension $\widehat{\Gamma}$; i.e., from $\operatorname{LDB}(\mathbf{V})$ to $\operatorname{DB}(\mathbf{V})$. Since $\widehat{\Gamma}$ is an order-based view, the results of [Heg04a] guarantee that the updates on $\widehat{\Gamma}$ are unique, and so these translate back to $\Gamma$. The upshot is that all updates on a CFA-view $\Gamma$ have a unique translation back to the main schema, provided that they are defi ned by a constant-complement strategy with some CFA-view $\Gamma^{\prime}$ as complement.

Due to space and time restrictions, it was not possible to provide a suitable elaboration to PFschemata and PF-views in this paper. These uniqueness results will be extended and reported separately.

7.3 Relationship to other work The problem of characterizing the complexity of the constraints on a view does not appear to have received much attention previously, aside from the classical work of Hull [Hul84], in which it is shown that fi nite specifi cation is not preserved under projection, and the work of Fagin [Fag82], which includes characterizations of projections of various classes of dependencies.

There has been some work on the complexity of verifying the acceptability of view updates under a constant complement strategy. In an early paper, Cosmadakis and Papadimitriou [CP84] present pessimistic complexity results for view update under constant complement which would appear to contradict those obtained here. However, they work with general subdirect complements, and not meet complements, and so their results do not apply to the closed update strategies considered here. They also investigate the complexity of identifying a minimal (not necessarily meet) complement which will support a given update, again with pessimistic results.

Recently, Lechtenbörger and Vossen [LV03] have also looked at the complexity of the problem of identifying (not necessarily meet) complements to views, but for the purpose of identifying information missing in the view, and not with an eye towards update strategies. Their approach, by design, does not concern itself with meet complements or update strategies. Beyond those works, most of the literature on the problem of complexity of view updates is focused on logic databases. The fundamental issues which arise in that context (theory-oriented database models) are quite different from those of instance-oriented database models, and so a meaningful comparison is diffi cult at best.

\section{References}

[AHV95] Abiteboul, S., Hull, R., and Vianu, V., Foundations of Databases, Addison-Wesley, 1995.

[AHS90] Adámek, J., Herrlich, H., and Strecker, G., Abstract and Concrete Categories, WileyInterscience, 1990.

[BS81] Bancilhon, F. and Spyratos, N., "Update semantics of relational views," ACM Trans. Database Systems, 6(1981), pp. 557-575. 
[BV84a] Beeri, C. and Vardi, M. Y., "Formal systems for tuple and equality generating dependencies," SIAM J. Computing, 13(1984), pp. 76-98.

[BV84b] Beeri, C. and Vardi, M. Y., "A proof procedure for data dependencies," J. Assoc. Comp. Mach., 31(1984), pp. 718-741.

[CV85] Chandra, A. K. and Vardi, M. Y., "The implication problem for functional and inclusion dependencies is undedidable," SIAM J. Computing, 14(1985), pp. 671-677.

[CKV90] Cosmadakis, S., Kannelakis, P. C., and Vardi, M. Y., "Polynomial-time implication problems for unary inclusion dependencies," J. Assoc. Comp. Mach., 37(1990), pp. 15-46.

[CP84] Cosmadakis, S. and Papadimitriou, C., "Updates of relational views," J. Assoc. Comp. Mach., 31(1984), pp. 742-760.

[DP02] Davey, B. A. and Priestly, H. A., Introduction to Lattices and Order, Cambridge University Press, second edition, 2002.

[EN04] Elmasri, R. and Navathe, S. B., Fundamentals of Database Systems, Pearson Education, fourth edition, 2004.

[Fag82] Fagin, R., "Horn clauses and database dependencies," J. Assoc. Comp. Mach., 29(1982), pp. 952-985.

[FV83] Fagin, R. and Vardi, M. Y., "Armstrong databases for functional and inclusion dependencies," Info. Process. Lett., 16(1983), pp. 13-19.

[FGM*04] Foster, J. N., Greenwald, M. B., Moore, J. T., Pierce, B. C., and Schmitt, A., "Combinators for bi-directional tree transformations: A linguistic approach to the view update problem," Technical Report MS-CIS-04-15, Department of Computer Science, University of Pennsylvania, August 2004, to appear in POPL 2005.

[GJ82] Grant, J. and Jacobs, B. E., "On the family of generalized dependency constraints," JACM, 29(1982), pp. 986-997.

[Heg90a] Hegner, S. J., "Foundations of canonical update support for closed database views," in: Abiteboul, S. and Kanellakis, P. C., eds., ICDT'90, Third International Conference on Database Theory, Paris, France, December 1990, pp. 422-436, Springer-Verlag, 1990.

[Heg90b] Hegner, S. J., "Some open problems on view axiomatization," Bulletin of the EATCS, 40(1990), pp. 496-498.

[Heg93] Hegner, S. J., "Characterization of desirable properties of general database decompositions," Ann. Math. Art. Intell., 7(1993), pp. 129-195.

[Heg94] Hegner, S. J., "Unique complements and decompositions of database schemata," $J$. Comput. System Sci., 48(1994), pp. 9-57. 
[Heg02] Hegner, S. J., "Uniqueness of update strategies for database views," in: Foundations of Information and Knowledge Systems: Second International Symposium, FoIKS 2002, Salzau Castle, Germany, February 2002, Proceedings, pp. 230-249, Springer-Verlag, 2002.

[Heg04a] Hegner, S. J., "An order-based theory of updates for database views," Ann. Math. Art. Intell., 40(2004), pp. 63-125.

[Heg04b] Hegner, S. J., "The relative complexity of updates for a class of database views," in: Seipel, D. and Turull-Torres, J. M., eds., Foundations of Information and Knowledge Systems: Third International Symposium, FoIKS 2004, Wilehminenberg Castle, Austria, February 17-20, 2004, Proceedings, pp. 155-175, Springer-Verlag, 2004.

[HSR98] Horowitz, E., Sahni, S., and Rajasekaran, S., Computer Algorithms, Computer Science Press, 1998.

[Hul84] Hull, R., "Finitely specifi able implicational dependency families," J. Assoc. Comp. Mach., 31(1984), pp. 210-226.

[JAK82] Jacobs, B. E., Aronson, A. R., and Klug, A. C., "On interpretations of relational languages and solutions to the implied constraint problem," ACM Transactions on Database Systems, 7(1982), pp. 291-315.

[LV03] Lechtenbörger, J. and Vossen, G., "On the computation of relational view components," ACM Trans. Database Systems, 28(2003), pp. 175-208.

[PDGV89] Paredaens, J., De Bra, P., Gyssens, M., and Van Gucht, D., The Structure of the Relational Database Model, Springer-Verlag, 1989.

[Tha91] Thalheim, B., Dependencies in Relational Databases, Volume 126 of Teubner-Texte zur Mathematik, Teubner, 1991.

[WG92] Wang, K. and Graham, M. H., "Constant-time maintainability: A generalization of independence,” ACM Trans. Database Systems, 17(1992), pp. 201-246.

\section{Appendix A: A Simple Counterexample to the Finite Axiomatiz- ability of Relational Views}

It is part of the folklore of the theory of relational databases that there exists a schema with a simple axiomatization which has a projective view which is not fi nitely axiomatizable. From time to time, it is useful to be able to point to such an example, complete with an explanation of why fi nite axiomatizability fails. Unfortunately, they have rarely made it into the literature. Only two are known to the author. In [Hul84, Lemma 4.1], Hull presents an example of a schema with fi ve attributes constrained by three functional dependencies (FDs). The view which is not fi nitely axiomatizable is a projection onto four of those attributes. In [Heg90b], the author identifi es a simpler example containing just four attributes and constrained by three FDs, with the corresponding view a projection onto three of those attributes. Unfortunately, that example contains an error; and, in any case, no argument for its 
validity is offered. In this note, the example of [Heg90b] is corrected, and the proof of the lack of fi nite axiomatizability of the view is elaborated.

$$
\left[\begin{array}{cccc}
a_{1} & b_{1} & c_{1} & d_{1} \\
a_{1} & b_{2} & c_{2} & d_{1} \\
a_{2} & b_{2} & c_{3} & d_{1} \\
a_{2} & b_{3} & c_{4} & d_{1} \\
a_{3} & b_{3} & c_{5} & d_{1} \\
a_{3} & b_{4} & c_{6} & d_{1} \\
a_{4} & b_{4} & c_{7} & d_{1} \\
a_{4} & b_{5} & c_{8} & d_{1} \\
\vdots & \vdots & \vdots & \vdots \\
a_{k-1} & b_{k-1} & c_{2 k-3} & d_{1} \\
a_{k-1} & b_{k} & c_{2 k-2} & d_{1} \\
\vdots & \vdots & \vdots & \vdots \\
a_{n-1} & b_{n-1} & c_{2 n-3} & d_{1} \\
a_{n-1} & b_{n} & c_{2 n-2} & d_{1} \\
a_{n} & b_{n} & c_{1} & d_{1}
\end{array}\right]
$$

Figure 4: The layout of the generic counterexample instance

Let $\mathbf{E}_{1}$ be the relational schema with the single relation name $R[A B C D]$ on four attributes; the constraining set of FDs is $\mathcal{F}_{1}=\{A \rightarrow D, B \rightarrow D, C D \rightarrow A\}$. The domain of possible values for each attribute is assumed to be infi nite. Let $\Pi_{A B C}=\left(R[A B C], \pi_{A B C}\right)$ denote the view which is the projection onto the attributes $A B C$. For any $n>0$, let $r(n)$ denote the instance which is depicted in Fig. 4. Assume that any two elements with distinct names are distinct values, save that $a_{1}$ and $a_{n}$ may be the same. It is easy to see that $r(n)$ is a legal instance of the main schema if and only if $a_{1}=a_{n}$. Similarly, $r^{\prime}(n)=\pi_{A B C}(r(n))$ is a legal instance of $\Pi_{A B C}$ under the implied constraints if and only if $a_{1}=a_{n}$, since a simple "chase" through any element of $\pi_{A B C}^{-1}\left(r^{\prime}(n)\right)$ shows that all of the values in the column of attribute $D$ must be the same. However, if any tuple from $r^{\prime}(n)$ is deleted, a valid instance of $R[A B C]$ is obtained even if $a_{1} \neq a_{n}$, since it is now possible to have two distinct values appearing in column $D$ in an inverse image. This situation is shown in Fig. 5 with the row containing $\left(a_{3}, b_{4}, c_{6}\right)$ deleted. In this case, it need not be the case that $a_{1}=a_{n}$. Therefore, $\Pi_{A B C}$ is not axiomatizable by any set of sentences having only $n$ free tuple variables (i.e.; a maximum of $n$ variables per column). Since $n$ is arbitrary, $\Pi_{A B C}$ is not fi nitely axiomatizable. In particular, it is not axiomatizable by any fi nite set of equality generating dependencies (EGDs) [AHV95, 10.1], much less by a family of functional dependencies. 


$$
\left[\begin{array}{cccc}
a_{1} & b_{1} & c_{1} & d_{1} \\
a_{1} & b_{2} & c_{2} & d_{1} \\
a_{2} & b_{2} & c_{3} & d_{1} \\
a_{2} & b_{3} & c_{4} & d_{1} \\
a_{3} & b_{3} & c_{5} & d_{1} \\
-e_{3}- & -b_{4} & --c_{6}- & --- \\
a_{4} & b_{4} & c_{7} & d_{2} \\
a_{4} & b_{5} & c_{8} & d_{2} \\
\vdots & \vdots & \vdots & \vdots \\
a_{k-1} & b_{k-1} & c_{2 k-3} & d_{2} \\
a_{k-1} & b_{k} & c_{2 k-2} & d_{2} \\
\vdots & \vdots & \vdots & \vdots \\
a_{n-1} & b_{n-1} & c_{2 n-3} & d_{2} \\
a_{n-1} & b_{n} & c_{2 n-2} & d_{2} \\
a_{n} & b_{n} & c_{1} & d_{2}
\end{array}\right]
$$

Figure 5: The layout of the generic counterexample instance with one row deleted 